\title{
Configurations of trivial extensions of Dynkin type $\mathbf{D}_{n}$
}

\author{
Octavio Mendoza Hernández \\ Instituto de Matemáticas \\ Universidad Nacional Autónoma de México. \\ Circuito Exterior, Ciudad Universitaria \\ C.P. 04510, México, D.F. MEXICO. \\ E-mail address: omendoza@matem. unam.mx
}

\begin{abstract}
For a given trivial extension $\Lambda$ of Cartan class $\mathbf{D}_{n}$, we find a combinatorial algorithm giving the configuration of $\mathbb{Z} \mathbf{D}_{n}$ associated to $\Lambda$; this algorithm is stated in the Theorem 6.8 and illustrated in the Example 6.3. In order to do that, we develop an inductive embedding technique for the Auslander-Reiten quiver $\Gamma_{\Lambda}$ of a trivial extension $\Lambda$ of type $\mathbf{D}_{n}$. This technique uses the ordinary quiver $Q_{\Lambda}$, which is the union of oriented cycles, and a well known set of relations $\rho$ such that $\Lambda$ is the quotient path algebra $k Q_{\Lambda} /\langle\rho>$ (see [1], [5] and [6]). Giving a trivial extension $\Gamma$ of type $\mathbf{D}_{N}$, we get a trivial extension $\Lambda$ of type $\mathbf{D}_{N+m-1}$ by inserting a cycle (with $m$ vertices) in one of the so called insertion vertices of $Q_{\Gamma}$. In Section 3, we describe the embedding of $\Gamma_{\Gamma}$ into $\Gamma_{\Lambda}$. This will allow us to deduce properties of $\Gamma_{\Lambda}$ from $\Gamma_{\Gamma}$; and since $Q_{\Gamma}$ has less cycles that $Q_{\Lambda}$, we will use this embedding to reduce the study of $\Gamma_{\Lambda}$ to the minimal trivial extensions, which were done in Section 2.
\end{abstract}

\section{Introduction}

In this paper, we only consider basic finite dimensional $k$-algebras with $k$ an algebraically closed field. In such a case, an algebra $A$ of this type is isomorphic to $k Q_{A} / I$, where $Q_{A}$ is the ordinary quiver associated to $A$ and $I$ is an admissible ideal of the path algebra $k Q_{A}$. For a quiver $Q$, the set of vertices will be denoted by $Q_{0}$ and the set

1991 Mathematics Subject Classification. Primary 16G70. Secondary 16D99. 
of arrows by $Q_{1}$.

Given a $k$-algebra $A$ and a vertex $j$ of $Q_{A}$, we denote by $S_{j}$ the simple $A$-module corresponding to the vertex $j$; and so, $P_{j}$ will denote the projective cover of $S_{j}$ and $I_{j}$ the injective envelope of $S_{j}$.

Let $A$ be an iterated tilted algebra of Dynkin type $\Delta$ (see in [2]); and let $T(A):=A \ltimes D_{A}(A)$ be the trivial extension of $A$ by its minimal injective cogenerator $D_{A}(A)=\operatorname{Hom}_{k}(A, k)$. The set of vertices $\left(\Gamma_{A}\right)_{0}$ of the Auslander-Reiten quiver $\Gamma_{A}$ of $A$ can be embedded in the stable part ${ }_{S} \Gamma_{T(A)}$ of the Auslander-Reiten quiver $\Gamma_{T(A)}$ of $T(A)$. Since $\mathbb{Z} \Delta \rightarrow{ }_{S} \Gamma_{T(A)}$ is the universal covering of ${ }_{S} \Gamma_{T(A)}$, the vertices of $\Gamma_{A}$ can be embedded in $\mathbb{Z} \Delta$ in such a way that knowing the vertices of $\mathbb{Z} \Delta$ corresponding to $A$-modules, we can obtain the arrows of $\Gamma_{A}$ (see in [8]). Hence, the quiver $\Gamma_{A}$ is embedded in $\mathbb{Z} \Delta$; and it is very useful for applications to describe this embedding explicitely. In [8], this problem was solved by assuming that the set of vertices in $\mathbb{Z} \Delta$ corresponding to the radicals of the indecomposable projective $T(A)$-modules is known. So, to have a complete description of this embedding, it is necessary to give an algorithm to determine which subsets of vertices in $\mathbb{Z} \Delta$ represent the radicals of the indecomposable projective modules over a given trivial extension $T$ of Dynkin type $\Delta$. These kind of vertices of $\mathbb{Z} \Delta$ have been considered before by Chr. Riedtmann, who called them configurations, in a more general setting $[4,10,11,12]$. The configurations of selfinjective algebras of finite type were computed in these works. One could use the results for selfinjective algebras and then decide which configurations correspond to a given trivial extension $\Lambda$. With a different approach, we present here a new algorithm giving directly the configuration of a given trivial extension of Dynkin type $\mathbf{D}_{n}$. The $\mathbf{A}_{n}$ case was solved in [9]. In what follows, we summarize the content of the paper.

Section 1: By following [5], we give (see Proposition 1.8) a presentation of the trivial extensions $\Lambda$ of Cartan class $\mathbf{D}_{N}$; and also we recall the lifting procedure of ${ }_{S} \Gamma_{\Lambda}$ to $\mathbb{Z} \mathbf{D}_{N}$ as was settled in $[8,9]$.

Section 2: Let $\Lambda$ be a trivial extension of Cartan class $\mathbf{D}_{N}$ and $\pi: \mathbb{Z D}_{N} \rightarrow{ }_{S} \Gamma_{\Lambda}$ be the universal covering of translation quivers. Consider the subset $\mathcal{C}_{\Lambda}:=\left\{r P_{i}: i \in\left(Q_{\Lambda}\right)_{0}\right\}$ of ${ }_{S} \Gamma_{\Lambda}$ representing the radicals of the indecomposable projective $\Lambda$-modules $P_{i}$ for $i \in$ $\left(Q_{\Lambda}\right)_{0}$, and the set $\widetilde{\mathcal{C}_{\Lambda}}=\pi^{-1}\left(\mathcal{C}_{\Lambda}\right)$. In this section, we describe the configuration $\widetilde{\mathcal{C}_{\Lambda}}$ associated to a given minimal trivial extension $\Lambda$ of 
type $\mathbf{D}_{N}$. We start by studying, under very special situations, how $r P_{j}$ can be lifted to $\mathbb{Z} \mathbf{D}_{N}$ for some $j \in\left(Q_{\Lambda}\right)_{0}$. We do this first for arbitrary trivial extensions of $\mathbf{D}_{N}$ type, and then specialize for the different minimal types defined in the previous section.

Section 3: Let $\Gamma$ be a trivial extension of Cartan class $\mathbf{D}_{N}, z$ an insertion vertex of $Q_{\Gamma}$ (see Definition 2.1) and $\Lambda$ be the trivial extension of Cartan class $\mathbf{D}_{N+m-1}$ obtained from $\Gamma$ by inserting the cycle $C_{z}=z \leftarrow z_{1} \leftarrow z_{2} \leftarrow \cdots \leftarrow z_{m-1} \leftarrow z$ at $z$. In this section, we study the embedding of ${ }_{S} \Gamma_{\Gamma}$ in ${ }_{S} \Gamma_{\Lambda}$. We also study the behavior of the irreducible morphisms of $\underline{\bmod } \Gamma$ inside $\underline{\bmod } \Lambda$ using the embedding $\imath: \underline{\bmod } \Gamma \rightarrow \underline{\bmod } \Lambda$.

Section 4: In the previous section, we described an embedding of ${ }_{S} \Gamma_{\Gamma}$ in ${ }_{S} \Gamma_{\Lambda}$. In this section, we study the corresponding embedding between their coverings $k\left(\mathbb{Z} \mathbf{D}_{N}\right)$ and $k\left(\mathbb{Z} \mathbf{D}_{N+m-1}\right)$. Here, the main results are the Theorem 4.11 and its Corollary 4.12.

Section 5: In this section, we extend the Proposition 2.14 to the general case, that is, to a trivial extension $\Lambda$ of Class II (see Definition 1.2 and Remark 1.9). To do that, we firstly consider the case when $\Lambda$ is minimal of Class II (see 2.14). Then, by using 4.11, we see how the radicals of the projective modules, associated with the vertices 1,2 and 3 of $Q_{\Lambda}$, can be lifted to the universal covering. Furthermore, we also determine some special regions, in the bottom border of $\mathbb{Z} \mathbf{D}_{N}$, where the radicals of the projective modules associated with the insertion vertices of $Q_{\Lambda}$ can be lifted.

Section 6: Let $\Lambda$ be a trivial extension of Cartan class $\mathbf{D}_{N}$. Consider the universal covering $\pi: \mathbb{Z} \mathbf{D}_{N} \rightarrow{ }_{S} \Gamma_{\Lambda}$ of ${ }_{S} \Gamma_{\Lambda}$. In this section, we give an algorithm to determine the configuration $\widetilde{\mathcal{C}_{\Lambda}}$ of $\mathbb{Z} \mathbf{D}_{N}$ associated to $\Lambda$. We recall that $\widetilde{\mathcal{C}_{\Lambda}}:=\pi^{-1}\left(\mathcal{C}_{\Lambda}\right)$, where $\mathcal{C}_{\Lambda}$ is the set of vertices of ${ }_{S} \Gamma_{\Lambda}$ representing the radicals of the indecomposable projective $\Lambda$ modules (see $[8,2.7])$. We define a subset $r \mathcal{P}\left(\Lambda, \mathbb{Z} \mathbf{D}_{N}\right)$ of $\left(\mathbb{Z} \mathbf{D}_{N}\right)_{0}$ and prove that $r \mathcal{P}\left(\Lambda, \mathbb{Z} \mathbf{D}_{N}\right)[\mathbb{Z}]:=\cup_{i \in \mathbb{Z}} r \mathcal{P}\left(\Lambda, \mathbb{Z} \mathbf{D}_{N}\right)[i]$ is the desired configuration. In order to do that, we start this section by defining some notations needed to state the algorithm: the sections $\mathcal{S}_{x}^{+}$and $\mathcal{S}_{x}^{-}$, the initial vertices, and the functions border and height defined on the set of vertices of $Q_{\Lambda}$. The algorithm is stated in the Theorem 6.8 and illustrated in the Example 6.3. The remainder of the section is devoted to the proof of the Theorem 6.8. 


\section{Preliminaries}

We start this section by recalling well known notions about quivers. Let $Q$ be a quiver. We denote by $Q_{0}$ the set of vertices and by $Q_{1}$ the set of arrows of $Q$. For any arrow $\alpha$ of $Q_{1}$ we have that it starts at $o(\alpha)$ and ends at $e(\alpha)$. A path $p$ in the quiver $Q$ is either an oriented sequence of arrows $\alpha_{n} \cdots \alpha_{1}$ with $e\left(\alpha_{t}\right)=o\left(\alpha_{t+1}\right)$ for $1 \leq t<n$ or the symbol $e_{i}$ for $i \in Q_{0}$. We call the paths $e_{i}$ trivial paths and we define $o\left(e_{i}\right)=e\left(e_{i}\right)$. Given a nontrivial path $p=\alpha_{n} \cdots \alpha_{1}$ we define $o(p)=o\left(\alpha_{1}\right)$ and $e(p)=e\left(\alpha_{n}\right)$. If $\delta$ is a path in $Q$, we denote by $\underline{\delta}$ the support of $\delta$ in $Q$. Thus, $\underline{\delta}$ is a subquiver of $Q$ having as vertices and arrows those belonging to $\delta$. A nontrivial path $p$ is said to be an oriented cycle if $o(p)=e(p)$.

Let $\mathcal{C}=\alpha_{n} \alpha_{n-1} \cdots \alpha_{2} \alpha_{1}$ be an oriented cycle in $Q$. We call $\mathcal{C}$ minimal oriented cycle if the vertices $o\left(\alpha_{1}\right), o\left(\alpha_{2}\right), \cdots, o\left(\alpha_{n}\right)$ are pairwise different. It is said that $Q^{\prime}$ is a full subquiver of $Q$ in case $Q^{\prime}$ is a subquiver of $Q$ such that for all vertices $i, j \in Q^{\prime}$ we have that each arrow $i \stackrel{\alpha}{\rightarrow} j$ of $Q$ is also an arrow of $Q^{\prime}$. A full subquiver $Q^{\prime}$ of $Q$ is called convex if for any path $a_{0} \rightarrow a_{1} \rightarrow \cdots \rightarrow a_{t}$ in $Q$, with $a_{0}, a_{t} \in Q_{0}^{\prime}$, we have $a_{i} \in Q_{0}^{\prime}$ for all $i=0,1, \cdots, t$.

\subsection{A presentation of trivial extensions of type $\mathbf{D}_{n}$.}

Following [5], we know that any trivial extension $\Lambda$ of type $\mathbf{D}_{n}$, which is not minimal, can be obtained from one minimal $\Gamma$, of type $\mathbf{D}_{N}$, by inserting to $\Gamma$ a trivial extension of type $\mathbf{A}_{m}$ with $n=N+$ $m-1$. In this section, we explain such a construction and also give a presentation $\left(Q_{\Lambda}, I\right)$ of $\Lambda$, which will be used throughout all the paper.

Proposition 1.1. [5] Let $\Lambda$ be a trivial extension. Then, $\Lambda$ is of type $\mathbf{A}_{n}$, with $n>1$, if and only if the ordinary quiver $Q_{\Lambda}$ of $\Lambda$ satisfies the following conditions:

(i) $Q_{\Lambda}$ has $n$ vertices;

(ii) $Q_{\Lambda}$ is the union of oriented cycles and there are no loops in $Q_{\Lambda}$

(iii) any two minimal oriented cycles of $Q_{\Lambda}$, having different supports, meet in at most one vertex;

(iv) every vertex $i \in Q_{\Lambda}$ belongs to at most two different supports of minimal oriented cycles; 
(v) if $C_{1}, C_{2}, \cdots, C_{m}$ are minimal oriented cycles in $Q_{\Lambda}$ such that $\underline{C_{i}} \neq \underline{C_{j}}$ for $i \neq j$, and $\underline{C_{1}} \cap \underline{C_{2}} \neq \emptyset, \underline{C_{2}} \cap \underline{C_{3}} \neq \emptyset, \cdots, \underline{C_{m-1}} \cap$ $\overline{C_{m}} \neq \bar{\emptyset}$, then $\underline{C_{1}} \cap \underline{C_{m}}=\emptyset$.

In what follows, we give, following [5], the ordinary quivers $Q_{\Lambda}$ corresponding to the smallest trivial extensions $\Lambda$ of type $\mathbf{D}_{N}$.

Definition 1.2. [5] Let $\Lambda$ be a trivial extension of Cartan class $\mathbf{D}_{N}$. We say that $\Lambda$ is minimal if the ordinary quiver $Q_{\Lambda}$ of $\Lambda$ belongs to one of the following classes of quivers.

(a) Class $I$ with $N=4+n$.

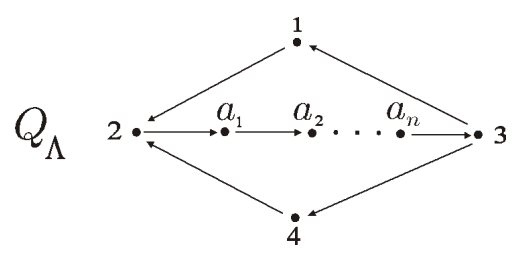

(b) Class II. This class is divided in 3 subclasses: $I I_{1}, I I_{2}$ and $\mathrm{II}_{3}$.

(b1) Subclass $I I_{1}$ with $N=3+n$.

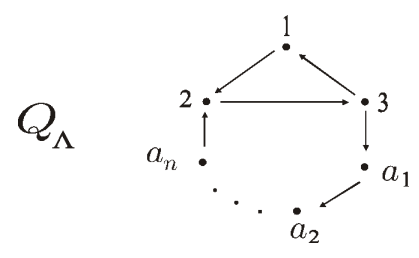

(b2) Subclass $I_{2}$ with $N=3+t+m$.

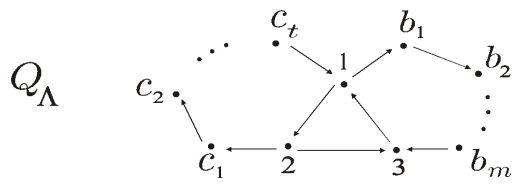

(b3) Subclass $I_{3}$ with $N=3+t+m+n$. 


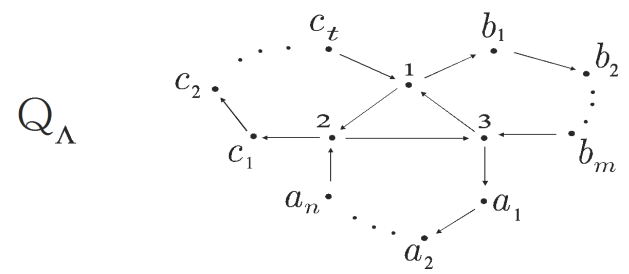

(c) Class III with $N=3+n$.

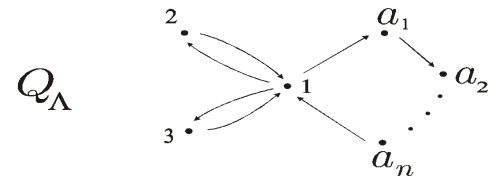

Remark 1.3. For the trivial extensions of class II, it will be convenient to relax the values of $m, n$ and $t$ to be zero. This means for example that if $m=t=0$ then $\Lambda$ is of class $I I_{1}$. Such a notation allows us to consider the subclasses $I I_{1}$ and $I I_{2}$ as particular cases of the subclass $\mathrm{II}_{3}$.

Definition 1.4. $[5,7]$ Let $\Lambda$ be a trivial extension of Dynkin Cartan class. By [5] (see also [7]), it is known that $Q_{\Lambda}$ is the union of oriented cycles. A minimal oriented cycle $C$ of $Q_{\Lambda}$ is said to be elemental if it is not zero in $\Lambda$.

The elemental cycles are very important to describe the trivial extensions of finite representation type as can be seen in the following result.

Theorem 1.5. [5] Let $\Lambda$ be a trivial extension of Dynkin Cartan class. Then $\Lambda \simeq k Q_{\Lambda} / I$, where the quiver $Q_{\Lambda}$ is the union of oriented cycles and the admissible ideal $I$ is generated by:

(i) the paths consisting of $n+1$ arrows in an elemental cycle of length $n$;

(ii) the paths whose arrows do not belong to a single elemental cycle;

(iii) the difference $q-q^{\prime}$, where $q$ and $q^{\prime}$ are paths starting and ending at the same vertices and such that there exists a path $\nu$ with $\nu q$ and $\nu q^{\prime}$ elemental cycles. 
Remark 1.6. Let $\Lambda$ be a trivial extension of Dynkin Cartan class $\Delta$. Then:

(1) To have a presentation $\left(Q_{\Lambda}, I\right)$ for $\Lambda$, it is enough to give the quiver $Q_{\Lambda}$ and the set $\left\{\underline{\mathcal{C}}_{1}, \cdots, \underline{\mathcal{C}}_{m}\right\}$ of supports of the elemental cycles in $Q_{\Lambda}$ since the admissible ideal $I$ is given by the above theorem.

(2) If $\Delta=\mathbf{A}_{n}$, then by [5], we have that the elemental cycles of $Q_{\Lambda}$ are the minimal oriented cycles.

(3) If $\Delta=\mathbf{D}_{n}$ and $\Lambda$ is minimal, we have by [5], that the elemental cycles of $Q_{\Lambda}$ are the minimal oriented cycles with one exception: the only minimal oriented cycle, which is not allowed to be elemental, is the minimal one formed by all the vertices of $Q_{\Lambda}$ for $\Lambda$ of Subclass $I_{3}$.

In order to get all the trivial extensions of type $\mathbf{D}_{N}$, by using minimal algebras, we need to add trivial extensions of type $\mathbf{A}_{k}$ to some special vertices in the minimal algebras. So, we recall from [5] this procedure.

Definition 1.7. [5] Let $\Gamma=k Q_{\Gamma} / I$ be a trivial extension with $I$ an admissible ideal of $k Q_{\Gamma}$. Let $a_{1}, a_{2}, \cdots, a_{m}$ be different vertices in $Q_{\Gamma}$ and $n_{1}, n_{2}, \cdots, n_{m}$ be natural numbers. Let $\Gamma^{\prime}:=k Q^{\prime} /\left(I \cap k Q^{\prime}\right)$ be a trivial extension, where $Q^{\prime}$ is a full subquiver of $Q_{\Gamma}$. For each $j=1,2, \cdots, m$, we consider a full subquiver $Q_{a_{j}}$ of $Q_{\Gamma}$ and set $\Gamma_{a_{j}}:=k Q_{a_{j}} /\left(I \cap k Q_{a_{j}}\right)$. We say that $\Gamma$ is obtained from $\Gamma^{\prime}$ by adding trivial extensions $\Gamma_{a_{j}}$ of Cartan type $\mathbf{A}_{n_{j}}$ to $a_{j}$ for $j=1,2, \cdots, m$, if the following conditions hold:

(i) for each $j, \Gamma_{a_{j}}$ is a trivial extension of Cartan type $\mathbf{A}_{n_{j}}$ with $n_{j} \geq 2$

(ii) $\left(Q_{\Gamma}\right)_{0}=Q_{0}^{\prime} \cup\left(Q_{a_{1}}\right)_{0} \cup \cdots \cup\left(Q_{a_{m}}\right)_{0}$; moreover, for each $j$, we have that

$Q_{0}^{\prime} \cap\left(Q_{a_{j}}\right)_{0}=\left\{a_{j}\right\} \quad$ and $\quad\left(Q_{a_{i}}\right)_{0} \cap\left(Q_{a_{j}}\right)_{0}=\emptyset \quad$ if $\quad i \neq j ;$

(iii) $I$ is generated by $I \cap k Q^{\prime}$ and $I \cap k Q_{a_{j}}$ for $j=1,2, \cdots, m$.

Example. Let $\Gamma$ be the trivial extension of Cartan class $\mathbf{D}_{6}$ with $Q_{\Gamma}$ the following quiver and $I$ the ideal considered in 1.5. 


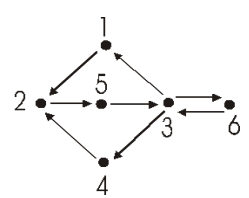

Consider the following full subquivers of $Q_{\Gamma}$,

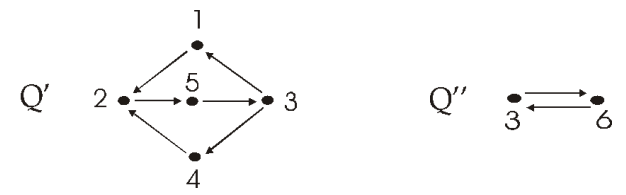

We have that $\Gamma^{\prime}:=k Q^{\prime} /\left(I \cap k Q^{\prime}\right)$ and $\Gamma^{\prime \prime}:=k Q^{\prime \prime} /\left(I \cap k Q^{\prime \prime}\right)$ are trivial extension of Cartan class $\mathbf{D}_{5}$ and $\mathbf{A}_{2}$, respectively. So, $\Gamma$ is obtained from $\Gamma^{\prime}$ by adding the trivial extension $\Gamma^{\prime \prime}$ of Cartan class $\mathbf{A}_{2}$ to the vertex 3 in $Q^{\prime}$.

The description of the trivial extensions of Cartan class $\mathbf{D}_{N}$ is given in the following result. Here we use the notation discussed in 1.3.

Proposition 1.8. [5] Let $\Lambda$ be a trivial extension of Cartan class $\mathbf{D}_{N}$. Then, $\Lambda$ is either minimal or can be obtained from a minimal one by adding trivial extensions of Cartan class $\mathbf{A}_{n_{j}}$ to some of the following vertices $x_{j}$ indicated with

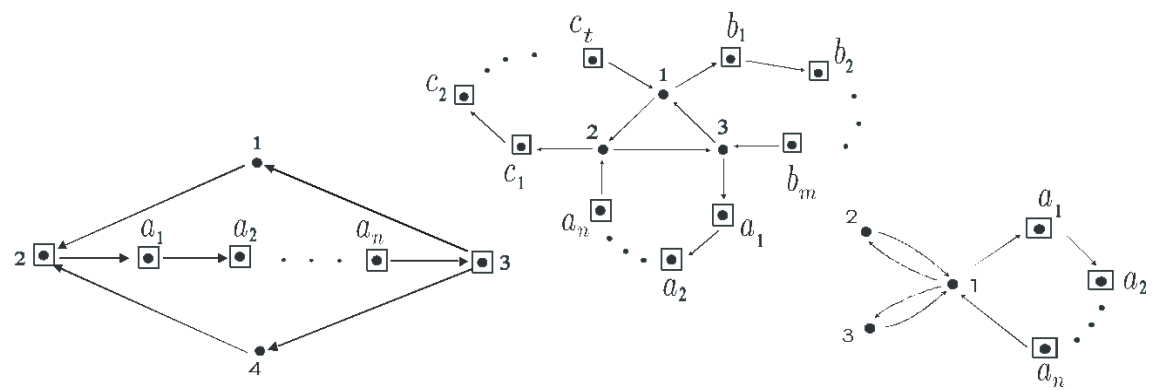

Remark 1.9. (1) We will denote by $\Lambda_{\text {min }}$ to the minimal trivial extension corresponding to $\Lambda$ in 1.8 .

(2) Any trivial extension $\Lambda$ of Cartan class $\mathbf{D}_{N}$ will be represented by one of the pictures given in 1.8. The little square on the vertex $x_{j}$ 
represents a quiver $Q_{x_{j}}$, which will be not drawn, where $Q_{x_{j}}$ is either $\left\{x_{j}\right\}$ or the ordinary quiver of a trivial extension $\Gamma_{x_{j}}$ of Cartan class $\mathbf{A}_{n_{j}}$ with $n_{j} \geq 2$. Moreover, we say that $\Lambda$ is of class $I, I I_{1}, I I_{2}, I I_{3}$ or III if $\Lambda_{\min }$ is so.

\subsection{Lifting ${ }_{S} \Gamma_{\Lambda}$ to $\mathbb{Z} \Delta$ having some $M \in \underline{\text { ind }} \Lambda$ as a source.}

Throughout this paper, we will use freely the notations that were settled in $[8,9]$. In particular, the lifting process defined in [8], will be crucial in the paper. For a given trivial extension $\Lambda$ of Dynkin Cartan class $\Delta$, a fixed object $M$ in ind $\Lambda$ and the universal covering $\pi: \mathbb{Z} \Delta \rightarrow{ }_{S} \Gamma_{\Lambda}$ of ${ }_{S} \Gamma_{\Lambda}$, where ${ }_{S} \Gamma_{\Lambda}$ is the stable AR-quiver of $\Lambda$, it was introduced in $\left[8\right.$, Section 3] the notion of the lifting of ${ }_{S} \Gamma_{\Lambda}$ to $\mathbb{Z} \Delta$ at the vertex $M$. Recall that this lifting procedure starts by fixing an element $M[0]$ in the fiber $\pi^{-1}(M)$; afterwards, we take a slice of ${ }_{S} \Gamma_{\Lambda}$ starting at $M$ and lift such slice through the universal covering $\pi: \mathbb{Z} \Delta \rightarrow{ }_{S} \Gamma_{\Lambda}$ to a unique slice of $\mathbb{Z} \Delta$ starting at $M[0]$. Analogously, the slice of ${ }_{S} \Gamma_{\Lambda}$ starting at $\tau^{-1}(M)$ can be lifted to a slice of $\mathbb{Z} \Delta$ starting at $\tau^{-1}(M[0])$. We iterate this procedure, the necessary number of times, lifting all the vertices of ${ }_{S} \Gamma_{\Lambda}$. The minimal connected subquiver of $\mathbb{Z} \Delta$, which contains all the lifted slices, is denoted by ${ }_{S} \Gamma_{\Lambda}[0]$ and will be called the lifting of ${ }_{S} \Gamma_{\Lambda}$ to $\mathbb{Z} \Delta$ at $M$. Then $\left.\pi\right|_{S \Gamma_{\Lambda}[0]}:{ }_{S} \Gamma_{\Lambda}[0] \rightarrow{ }_{S} \Gamma_{\Lambda}$ is a quiver morphism which is a bijection on the vertices of ${ }_{S} \Gamma_{\Lambda}[0]$. The inverse $\varphi_{M}:\left({ }_{S} \Gamma_{\Lambda}\right)_{0} \rightarrow(\mathbb{Z} \Delta)_{0}$ of this bijection, defines an embedding of ${ }_{S} \Gamma_{\Lambda}$ into $\mathbb{Z} \Delta$. For $X \in \underline{\text { ind }} \Lambda$ and $i \in \mathbb{Z}$, we denote by $X[i]$ the vertex $\tau^{-i m_{\Delta}} X[0]$ of $\mathbb{Z} \Delta$, where $X[0]:=\varphi_{M}(X)$ and $m_{\Delta}$ is the Loewy Length of the mesh category $k(\mathbb{Z} \Delta)$ (see [8, Section 3]). Furthermore, if we do not want to state precisely the lifting vertex, we will just say that ${ }_{S} \Gamma_{\Lambda}[0]$ is a lifting of ${ }_{S} \Gamma_{\Lambda}$ to $\mathbb{Z} \Delta$. We recall that $m_{\Delta}=2 N-3$ if $\Delta=\mathbf{D}_{N}$.

We introduce now the notions of height function and borders in $\mathbb{Z} \mathbf{D}_{N}$. To do that, we label the vertices of $\mathbf{D}_{N}$ as follows:

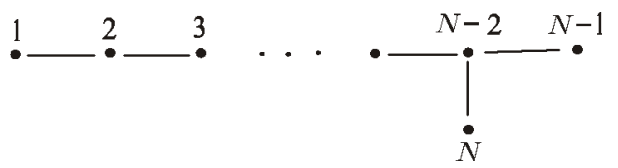


Definition 1.10. The height in $\mathbb{Z} \mathbf{D}_{N}$ is the function $h=h_{\mathbb{Z} \mathbf{D}_{N}}$ : $\left(\mathbb{Z D}_{N}\right)_{0} \rightarrow\{1,2, \cdots, N\}$ defined by $h_{\mathbb{Z} \mathbf{D}_{N}}(p, q):=q$. We distinguish 3 types of borders in $\mathbb{Z} \mathbf{D}_{N}:(a)$ the bottom border given by all the vertices of $\mathbb{Z} \mathbf{D}_{N}$ with height 1 , (b) the top border corresponds to the vertices of $\mathbb{Z} \mathbf{D}_{N}$ with height $N-1$ and $(c)$ the middle border consists of the vertices having height $N$.

The next picture shows which vertices of the section of $\mathbb{Z} \mathbf{D}_{N}$, starting at $(0,1)$, belong to the borders of $\mathbb{Z} \mathbf{D}_{N}$.

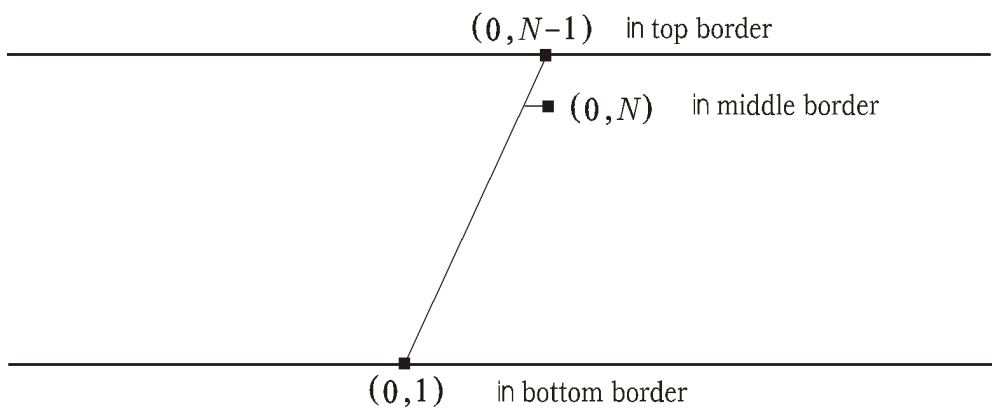

Let $\Lambda$ be a trivial extension of Cartan class $\mathbf{D}_{N}$ and $\pi: \mathbb{Z} \mathbf{D}_{N} \rightarrow$ ${ }_{S} \Gamma_{\Lambda}$ the universal covering of translation quivers. Consider the subset $\mathcal{C}_{\Lambda}:=\left\{r P_{i}: i \in\left(Q_{\Lambda}\right)_{0}\right\}$ of ${ }_{S} \Gamma_{\Lambda}$ representing the radicals of the indecomposable projective $\Lambda$-modules $P_{i}$ for $i \in\left(Q_{\Lambda}\right)_{0}$, and set $\widetilde{\mathcal{C}_{\Lambda}}=$ $\pi^{-1}\left(\mathcal{C}_{\Lambda}\right)$. From [10, 2.3 and 2.2], we know that $\widetilde{\mathcal{C}_{\Lambda}}$ is a configuration of $\mathbb{Z} \Delta$ and $\mathcal{C}_{\Lambda}$ is a configuration of ${ }_{S} \Gamma_{\Lambda}$. In this paper, we will define an algorithm to compute the vertices of $\mathbb{Z} \mathbf{D}_{N}$ corresponding to the configuration $\widetilde{\mathcal{C}_{\Lambda}}$ associated with $\Lambda$. The fundamental question to start working with is the following. Let $i \rightarrow j$ be an arrow of $Q_{\Lambda}$ and suppose that we know the position on $\mathbb{Z} \mathbf{D}_{N}$ of some element $r P_{j}[0]$ belonging to the fiber $\pi^{-1}\left(r P_{j}\right)$. Then, how does the radical $r P_{i}$ lifts to $\mathbb{Z} \mathbf{D}_{N}$ ? To answer this question, we will need to compute the support of the functors $k\left(\mathbb{Z} \mathbf{D}_{N}\right)(x,-)$ and $k\left(\mathbb{Z} \mathbf{D}_{N}\right)(-, x)$ for any vertex $x \in \mathbb{Z} \mathbf{D}_{N}$. We will denote by $\operatorname{Supp} F$ the support of a given functor $F$. Also, for the sake of simplicity, we set $\operatorname{Supp}(x,-):=\operatorname{Supp} k\left(\mathbb{Z} \mathbf{D}_{N}\right)(x,-)$ and $\operatorname{Supp}(-, x):=\operatorname{Supp} k\left(\mathbb{Z} \mathbf{D}_{N}\right)(-, x)$ whenever it is clear from the 
context which functor is being considered. We recall that (see $[8$, Section 3]) the additive function $f_{x}:\left(\mathbb{Z}_{N}\right)_{0} \rightarrow \mathbb{Z}$, which has value 1 on the slice starting at $x$, determines the support of the functor $k\left(\mathbb{Z} \mathbf{D}_{N}\right)(x,-)$ since $\operatorname{dim}_{k} k\left(\mathbb{Z} \mathbf{D}_{N}\right)(x, y)=f_{x}(y)$. Moreover, it is known that $\operatorname{Supp} k\left(\mathbb{Z} \mathbf{D}_{N}\right)(x,-)=\operatorname{Supp} k\left(\mathbb{Z} \mathbf{D}_{N}\right)\left(-, \nu_{\mathbf{D}_{N}}(x)\right)$, where $\nu_{\mathbf{D}_{N}}(x)$ is the Nakayama permutation. So, by using the additive function $f_{x}$, it can be seen that the support of the functors we are interested in has the following shape.

Remark 1.11. The shaded regions of the pictures below represent the support of the functor $k\left(\mathbb{Z} \mathbf{D}_{N}\right)(x,-)$.

(a) For $1 \leq h_{\mathbb{Z} \mathbf{D}_{N}}(x) \leq N-2$ :

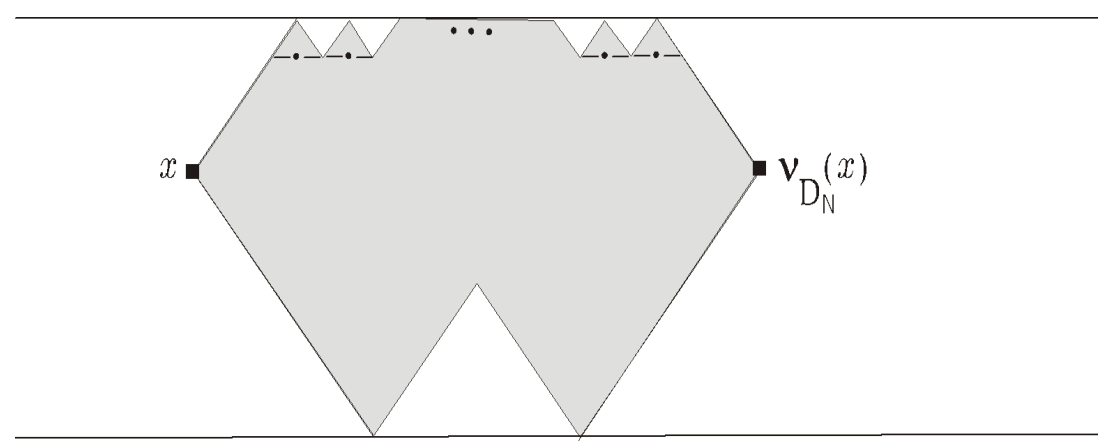

In particular, if $x$ is in the bottom border of $\mathbb{Z} \mathbf{D}_{N}$, we obtain a triangle with $x$ and $\nu_{\mathbf{D}_{N}}(x)$ in the bottom border.

(b) For $h_{\mathbb{Z} \mathbf{D}_{N}}(x)=N-1$ :

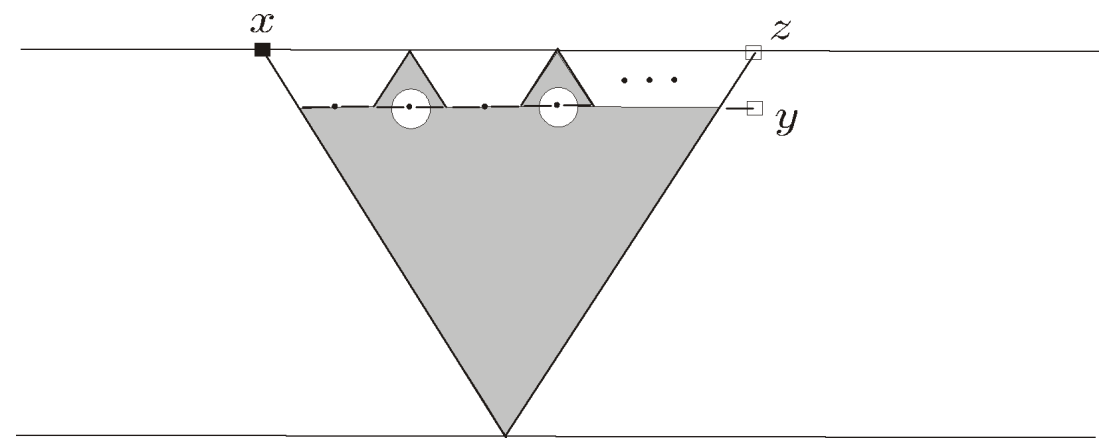




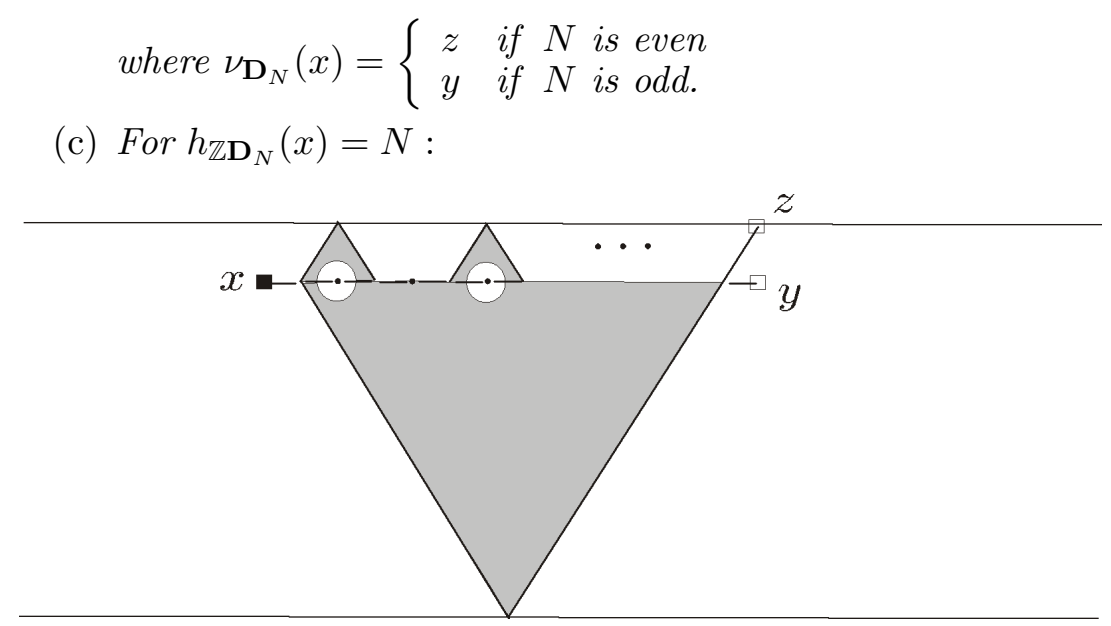

where $\nu_{\mathbf{D}_{N}}(x)= \begin{cases}y & \text { if } N \text { is even } \\ z & \text { if } N \text { is odd. }\end{cases}$

Proposition 1.12. Let $\Lambda$ be a trivial extension of Cartan class $\mathbf{D}_{N}$, $i \rightarrow j$ an arrow in $Q_{\Lambda}$ and ${ }_{S} \Gamma_{\Lambda}[0]$ a lifting of ${ }_{S} \Gamma_{\Lambda}$ to $\mathbb{Z}_{N}$ at $r P_{j}$. Then

(a) $r P_{i}[0] \in \operatorname{Supp}\left(\tau^{-1} r P_{j}[0],-\right) \backslash \operatorname{Supp}\left(r P_{j}[0],-\right)$;

(b) if $r P_{i} \simeq P_{j} / \operatorname{soc} P_{j}$, then $\tau^{-1} r P_{j}[0]=r P_{i}[0]$ and $r P_{j}[0]$ belongs to some border of $\mathbb{Z} \mathbf{D}_{N}$.

Proof. (a) By [9, Prop. 3.4], we get that $r P_{i}[0] \in \operatorname{Supp}\left(\tau^{-1} r P_{j}[0],-\right)$. On the other hand $k\left(\mathbb{Z} \mathbf{D}_{N}\right)\left(r P_{j}[0], r P_{i}[0]\right)=0$ since $Q_{\Lambda}$ has no loops (see 1.8 and [9, Proposition 1.1]).

(b) Let $r P_{i} \simeq P_{j} / \operatorname{soc} P_{j}$. Thus $\tau^{-1} r P_{j} \simeq r P_{i}$. On the other hand, in order to see that $r P_{j}[0]$ belongs to some border of $\mathbb{Z} \mathbf{D}_{N}$, it is enough to prove that $r P_{j} / \operatorname{soc} P_{j}$ is indecomposable. To do that, we will use; firstly, the description of $Q_{\Lambda}$ given in Subsection 1.1; and secondly, a combinatorial description of the number of the indecomposable direct summands of $r P_{j} / \operatorname{soc} P_{j}$ which is given in [6, Corollary 4.12].

Suppose that $r P_{j} / \operatorname{soc} P_{j}$ has at least two indecomposable direct summands. Then, from [6, Corollary 4.12], we get the existence of two elemental cycles $C$ and $C^{\prime}$ in $Q_{\Lambda}$ such that: (i) $\alpha: i \rightarrow j$ is in 
$C$ but not in $C^{\prime}$ and (ii) there is an arrow $\beta: j \rightarrow t$ which is in $C^{\prime}$ but not in $C$. Moreover, by [9, Proposition 1.1] and 1.8, we have that the cycles $C$ and $C^{\prime}$ have exactly one common vertex: the vertex $j$. Therefore, from 1.5, we conclude that the path $\beta \alpha$ is zero in $\Lambda$. Hence, the simple $S_{t}$, associated with the vertex $t$, is a composition factor of $P_{j} / \operatorname{soc} P_{j}$ which is not a composition factor of $r P_{i}$; giving a contradiction since $P_{j} / \operatorname{soc} P_{j} \simeq r P_{i}$.

We will need to know the vertices of $\operatorname{Supp}\left(\tau^{-1} r P_{j}[0],-\right) \backslash$ $\operatorname{Supp}\left(r P_{j}[0],-\right)$ in case $r P_{j}[0]$ is either on the top or in the middle border of $\mathbb{Z} \mathbf{D}_{N}$. These vertices can be easily computed by 1.11 .

Remark 1.13. Suppose that $r P_{j}[0]$ is either on the top or in the middle border of $\mathbb{Z} \mathbf{D}_{N}$. In the picture below, we have indicated with $\square$ the vertices of

$$
\operatorname{Supp}\left(\tau^{-1} r P_{j}[0],-\right) \backslash \operatorname{Supp}\left(r P_{j}[0],-\right)
$$

in case $h_{\mathbb{Z} \mathbf{D}_{N}}\left(r P_{j}[0]\right)=N-1\left(\right.$ for $h_{\mathbb{Z} \mathbf{D}_{N}}\left(r P_{j}[0]\right)=N$, the pictures to be considered are essentially the same).
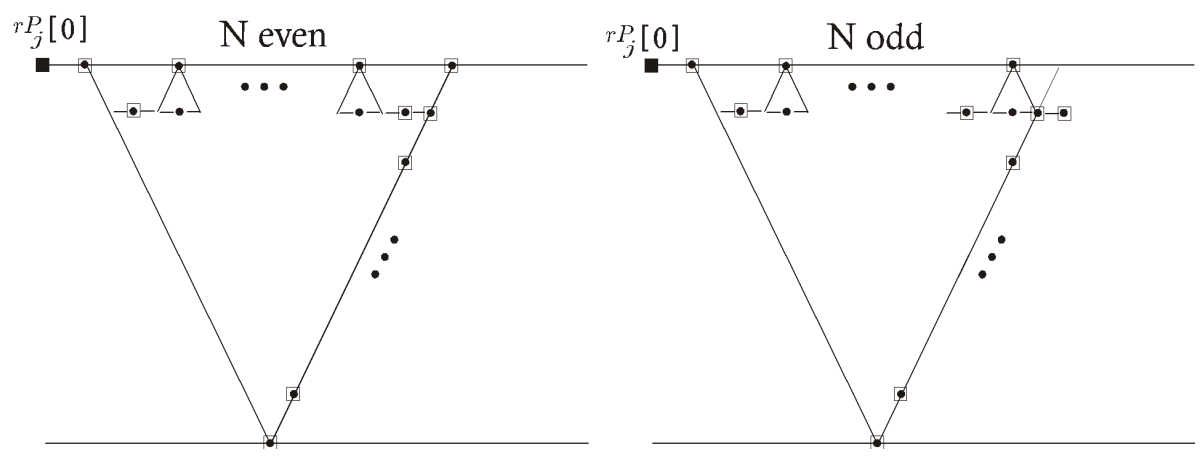

\section{Lifting some vertices of ${ }_{S} \Gamma_{\Lambda}$ to $\mathbb{Z}_{N}$ for trivial exten- sions $\Lambda$}

Let $\Lambda$ be a trivial extension of Cartan class $\mathbf{D}_{N}$ and $\pi: \mathbb{Z} \mathbf{D}_{N} \rightarrow$ ${ }_{S} \Gamma_{\Lambda}$ be the universal covering of translation quivers. Consider the subset $\mathcal{C}_{\Lambda}:=\left\{r P_{i}: i \in\left(Q_{\Lambda}\right)_{0}\right\}$ of ${ }_{S} \Gamma_{\Lambda}$ representing the radicals of the indecomposable projective $\Lambda$-modules $P_{i}$ for $i \in\left(Q_{\Lambda}\right)_{0}$, and the 
set $\widetilde{\mathcal{C}_{\Lambda}}=\pi^{-1}\left(\mathcal{C}_{\Lambda}\right)$. In this section, we will describe the configuration $\widetilde{\mathcal{C}_{\Lambda}}$ associated to a given minimal trivial extension $\Lambda$ of type $\mathbf{D}_{N}$. We start by studying, under very special situations, how $r P_{j}$ can be lifted to $\mathbb{Z} \mathbf{D}_{N}$ for some $j \in\left(Q_{\Lambda}\right)_{0}$. We do this first for arbitrary trivial extensions of $\mathbf{D}_{N}$ type, and then specialize for the different minimal types defined in the previous section.

\subsection{Insertion vertices in $Q_{\Lambda}$.}

Definition 2.1. [8] Let $\Lambda$ be a trivial extension of Cartan class $\mathbf{D}_{N}$ and $\Gamma$ be a trivial extension of Cartan class $\mathbf{D}_{k}$. If $C$ is an elemental cycle of $Q_{\Lambda}, Q_{\Gamma}$ is the union of the remaining cycles of $Q_{\Lambda}$ and $C \cap Q_{\Gamma}$ is a single vertex $z$, we say that $C$ is an elimination cycle of $Q_{\Lambda}$ and that $\Gamma$ is obtained from $\Lambda$ by eliminating the cycle $C$. Also we say that $\Lambda$ is obtained from $\Gamma$ by inserting the cycle $C$ at $z$.

$A$ vertex $x$ of $Q_{\Gamma}$, where a minimal oriented cycle $C$ can be inserted in order to obtain a trivial extension $\Lambda$ of Cartan class $\mathbf{D}_{N}$ with $N>k$, is called insertion vertex.

Lemma 2.2. For a trivial extension $\Lambda$ of Cartan class $\mathbf{D}_{N}$, the following statements hold.

(a) Let $\gamma=z_{1} \rightarrow z_{2} \rightarrow \cdots \rightarrow z_{\ell-1} \rightarrow z_{\ell}$ be a path belonging to a single elemental cycle of $Q_{\Lambda}$. If $z_{1}, z_{2}, \cdots, z_{\ell}$ are insertion vertices, then

$$
P_{z_{\ell}} / \operatorname{soc} P_{z_{\ell}} \simeq r P_{z_{\ell-1}}, \cdots, P_{z_{2}} / \operatorname{soc} P_{z_{2}} \simeq r P_{z_{1}} .
$$

(b) If $\Lambda$ is of class $I$ then $r P_{1} / \operatorname{soc} P_{1} \simeq r P_{4} / \operatorname{soc} P_{4}$.

(c) If $\Lambda$ is of class II then $r P_{i} / \operatorname{soc} P_{i}$ is indecomposable for any $i=1,2,3$. Moreover,

(c1) For $\Lambda$ of class $I I_{1}$, we have that $P_{3} / \operatorname{soc} P_{3} \simeq r P_{2}$. Furthermore,

$r P_{1} / \operatorname{soc} P_{1} \simeq r P_{a_{1}} / \operatorname{soc} P_{a_{1}}$ if $N=4 ;$ and in case $N>4$, $r P_{1} / \operatorname{soc} P_{1} \not r P_{a_{n}} / \operatorname{soc} P_{a_{n}}$.

(c2) If $\Lambda$ is either of class $I_{2}$ or $I I_{3}$, then

(i) $r P_{c_{i}} / \operatorname{soc} P_{c_{i}} \not f r P_{3} / \operatorname{soc} P_{3}$ for any $i=1,2, \cdots, t$;

(ii) $r P_{b_{j}} / \operatorname{soc} P_{b_{j}} \not r P_{2} / \operatorname{soc} P_{2}$ for any $j=1,2, \cdots, m$.

(d) For $\Lambda$ of class III, we have that $r P_{2} / \operatorname{soc} P_{2} \simeq S_{1} \simeq r P_{3} / \operatorname{soc} P_{3}$ and $r P_{1} / \operatorname{soc} P_{1} \simeq S_{2} \oplus S_{3} \oplus M$, where $M$ is a uniserial $\Lambda$ module which has $S_{a_{1}}, S_{a_{2}}, \cdots, S_{a_{n}}$ as composition factors. 
Proof. It can be proven by using the description of $Q_{\Lambda}$ given in 1.8 and the generators of the admissible ideal $I$ given in 1.5 .

Lemma 2.3. Let $\Lambda$ be a trivial extension of Cartan class $\mathbf{D}_{N}$, and let $i, j$ be different vertices of $Q_{\Lambda}$. In the case in which $r P_{i} / \operatorname{soc} P_{i}$ is indecomposable, we consider a lifting ${ }_{S} \Gamma_{\Lambda}[0]$ of ${ }_{S} \Gamma_{\Lambda}$ to $\mathbb{Z}_{N}$ at $r P_{i} / \operatorname{soc} P_{i}$.

(a) If $r P_{i} / \operatorname{soc} P_{i} \simeq r P_{j} / \operatorname{soc} P_{j}$ then $r P_{i} / \operatorname{soc} P_{i}$ is indecomposable. Moreover, there exist arrows $r P_{j}[-1] \rightarrow r P_{i} / \operatorname{soc} P_{i}[0] \leftarrow r P_{i}[-1]$ in $\mathbb{Z} \mathbf{D}_{N}$. Furthermore, $h_{\mathbb{Z} \mathbf{D}_{N}}\left(r P_{i} / \operatorname{soc} P_{i}[0]\right)=N-2$; and, in case $N>4$, we have that $h_{\mathbb{Z} \mathbf{D}_{N}}\left(r P_{t}[-1]\right)>N-2$ for $t=i, j$.

(b) If $r P_{i} / \operatorname{soc} P_{i}$ and $r P_{j} / \operatorname{soc} P_{j}$ are indecomposable and there is an arrow $r P_{j}[-1] \rightarrow r P_{i} / \operatorname{soc} P_{i}[0]$ in $\mathbb{Z} \mathbf{D}_{N}$, then $r P_{i} / \operatorname{soc} P_{i} \simeq$ $r P_{j} / \operatorname{soc} P_{j}$.

Proof. We recall that, if $P$ is a projective and injective indecomposable $\Lambda$-module then $0 \rightarrow r P \rightarrow r P / \operatorname{soc} P \oplus P \rightarrow P / \operatorname{soc} P \rightarrow 0$ is an Auslander-Reiten sequence in $\bmod \Lambda$.

(a) Since $r P_{i} / \operatorname{soc} P_{i} \simeq r P_{j} / \operatorname{soc} P_{j}$, we obtain that there exist irreducible morphisms $r P_{i} \rightarrow r P_{i} / \operatorname{soc} P_{i}$ and $r P_{j} \rightarrow r P_{i} / \operatorname{soc} P_{i}$. Suppose that $r P_{i} / \operatorname{soc} P_{i}$ has at least two non-isomorphic indecomposable direct summands $X$ and $Y$. Then, the quiver of Figure 1 below is a full subquiver of ${ }_{S} \Gamma_{\Lambda}$.
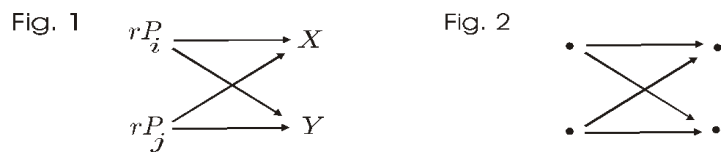

On the other hand, it is known that ${ }_{S} \Gamma_{\Lambda} \simeq \mathbb{Z} \mathbf{D}_{N} /<\tau^{2 N-3}>$ (see [1] and [4]). Hence, $r P_{i} / \operatorname{soc} P_{i}$ is indecomposable since the quiver of the Figure 2 is not a full subquiver of $\mathbb{Z} \mathbf{D}_{N}$.

Using that there is an irreducible morphism $r P_{j} \rightarrow r P_{i} / \operatorname{soc} P_{i}$, we get an arrow $r P_{t}[-1] \rightarrow r P_{i} / \operatorname{soc} P_{i}[0]$ in $\mathbb{Z} \mathbf{D}_{N}$ for $t=i, j$. On the other hand, since $r P_{i} / \operatorname{soc} P_{i} \simeq r P_{j} / \operatorname{soc} P_{j}$, by using the shape of $\mathbb{Z} \mathbf{D}_{N}$, it follows that $h_{\mathbb{Z} \mathbf{D}_{N}}\left(r P_{i} / \operatorname{soc} P_{i}[0]\right)=N-2$ and $h_{\mathbb{Z} \mathbf{D}_{N}}\left(r P_{t}[-1]\right)>N-2$ for $t=i, j$; proving (a). 
(b) Let $F: k\left(\mathbb{Z} \mathbf{D}_{N}\right) \rightarrow$ ind $\Lambda$ be a well-behaved functor which is induced by the universal covering $\pi: \mathbb{Z} \mathbf{D}_{N} \rightarrow{ }_{S} \Gamma_{\Lambda}$ (see [10, 1.5]). Suppose that $r P_{j} / \operatorname{soc} P_{j}$ is indecomposable and that there exists an arrow $\alpha: r P_{j}[-1] \rightarrow r P_{i} / \operatorname{soc} P_{i}[0]$ in $\mathbf{Z D}_{N}$. Then $F(\bar{\alpha}): r P_{j} \rightarrow r P_{i} / \operatorname{soc} P_{i}$ is irreducible. Therefore $r P_{i} / \operatorname{soc} P_{i}$ is a direct summand of $r P_{j} / \operatorname{soc} P_{j}$. Thus $r P_{i} / \operatorname{soc} P_{i} \simeq r P_{j} / \operatorname{soc} P_{j}$ since $r P_{j} / \operatorname{soc} P_{j}$ is indecomposable.

\subsection{Lifting $r P_{j}$ to $\mathbb{Z} \mathbf{D}_{N}$ under certain general conditions.}

Lemma 2.4. Let $\Lambda$ be a trivial extension of Cartan class $\mathbf{D}_{N}$, and $i \rightarrow t \rightarrow j$ be arrows of $Q_{\Lambda}$ such that $i \neq j$.

(a) If $P_{j} / \operatorname{soc} P_{j} \simeq r P_{t}$ then $r P_{j} / \operatorname{soc} P_{j}$ is indecomposable. Moreover, for any lifting of ${ }_{S} \Gamma_{\Lambda}$ to $\mathbb{Z} \mathbf{D}_{N}$ at $r P_{j} / \operatorname{soc} P_{j}$, we get that: $r P_{j}[-1]$ belongs to some border of $\mathbb{Z} \mathbf{D}_{N}, \tau^{-1} r P_{j}[-1]=r P_{t}[0]$ and

$r P_{i}[0] \in \operatorname{Supp}\left(\tau^{-1} r P_{t}[0],-\right) \backslash\left(\operatorname{Supp}\left(r P_{t}[0],-\right) \cup \operatorname{Supp}\left(r P_{j}[-1],-\right)\right)$.

(b) If $P_{j} / \operatorname{soc} P_{j} \simeq r P_{t}$ and $P_{t} / \operatorname{soc} P_{t} \simeq r P_{i}$, then $r P_{j}$ lifts to the bottom border of $\mathbb{Z} \mathbf{D}_{N}$.

Proof. (a) Let $P_{j} / \operatorname{soc} P_{j} \simeq r P_{t}$. By 1.12 (b), we have that $r P_{j}[-1]$ belongs to some border of $\mathbb{Z} \mathbf{D}_{N}$; and therefore, $r P_{j} / \operatorname{soc} P_{j}$ is indecomposable. On the other hand, $\tau^{-1} r P_{j}[-1]=r P_{t}[0]$ since $\tau^{-1} r P_{j}=$ $r P_{t}$. Moreover, since $i \rightarrow t$ is an arrow of $Q_{\Lambda}$, we get, by 1.12 (a), that $r P_{i}[0] \in \operatorname{Supp}\left(\tau^{-1} r P_{t}[0],-\right) \backslash \operatorname{Supp}\left(r P_{t}[0],-\right)$. Furthermore $k\left(\mathbb{Z} \mathbf{D}_{N}\right)\left(r P_{j}[-1], r P_{i}[0]\right)=0$ because $i \neq j$; proving (a).

(b) Let $P_{j} / \operatorname{soc} P_{j} \simeq r P_{t}$ and $P_{t} / \operatorname{soc} P_{t} \simeq r P_{i}$. From (a), we know that $r P_{j}$ lifts to some border of $\mathbb{Z} \mathbf{D}_{N}$. Let ${ }_{S} \Gamma_{\Lambda}[0]$ be a lifting of ${ }_{S} \Gamma_{\Lambda}$ to $\mathbb{Z} \mathbf{D}_{N}$ at $r P_{j}$; and suppose that $r P_{j}[0]$ is either in the middle or in the top border of $\mathbb{Z} \mathbf{D}_{N}$. Then, we get that $r P_{j}[0], r P_{t}[0]$ and $r P_{i}[0]$ are consecutive vertices either in the middle or in the top border of $\mathbb{Z} \mathbf{D}_{N}$. So, by 1.11 , we obtain that $k\left(\mathbb{Z} \mathbf{D}_{N}\right)\left(r P_{j}[0], r P_{i}[0]\right) \neq 0$; contradicting that $i \neq j$. Therefore $r P_{j}[0]$ is in the bottom border of $\mathbb{Z} \mathbf{D}_{N}$.

Remark 2.5. In case $i \rightarrow t \rightarrow j$ is a path in $Q_{\Lambda}$ with $i \neq j$ and $P_{j} / \operatorname{soc}_{j} \simeq r P_{t}$, we illustrate in the picture below the shape of the set

$$
\operatorname{Supp}\left(\tau^{-1} r P_{t}[0],-\right) \backslash\left(\operatorname{Supp}\left(r P_{t}[0],-\right) \cup \operatorname{Supp}\left(r P_{j}[-1],-\right)\right)
$$


for $h_{\mathbb{Z} \mathbf{D}_{N}}\left(r P_{j}[-1]\right)=N-1$. The vertices indicated with $\square$, in the following pictures, are the elements of such a set; and one of these vertices is $r P_{i}[0]$.
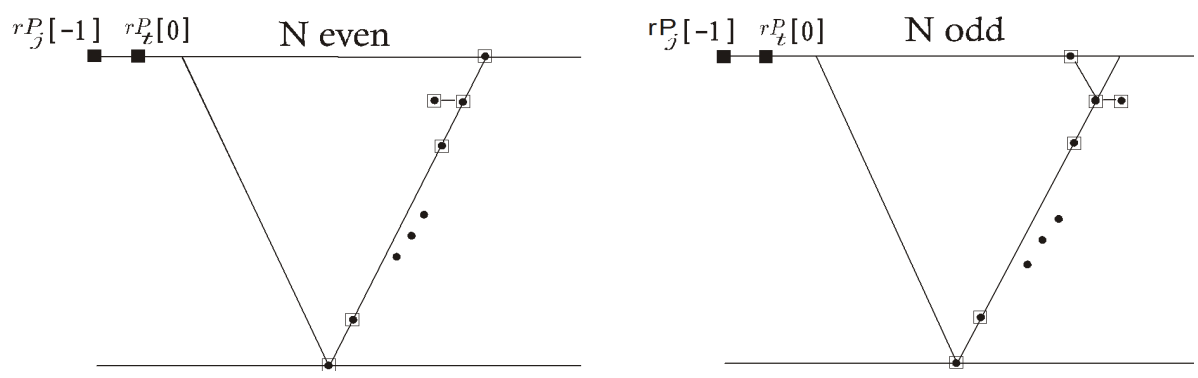

Lemma 2.6. Let $\Lambda$ be a trivial extension of Cartan class $\mathbf{D}_{N}$ with $N>4$; and let $\Lambda$ be such that either $i \leftarrow t \rightarrow j$ or $i \rightarrow t \leftarrow j$ is a subquiver of $Q_{\Lambda}$ with $r P_{i} / \operatorname{soc} P_{i} \simeq r P_{j} / \operatorname{soc} P_{j}$. Then, for any lifting of ${ }_{S} \Gamma_{\Lambda}$ to $\mathbb{Z} \mathbf{D}_{N}$ at $r P_{j} / \operatorname{soc} P_{j}$, there is an arrow $r P_{i}[-1] \rightarrow r P_{j} / \operatorname{soc} P_{j}[0]$ in $\mathbb{Z} \mathbf{D}_{N}$, where $r P_{i}[-1]$ is either in the middle or in the top border of $\mathbb{Z} \mathbf{D}_{N}$.

In the pictures below, we indicated with $\square$ the possible position of $r P_{t}[0]$ (respectively $r P_{t}[-1]$ ) in $\mathbb{Z} \mathbf{D}_{N}$.

(a) $r P_{t}[0]:$ In case $i \leftarrow t \rightarrow j$ is a subquiver of $Q_{\Lambda}$.

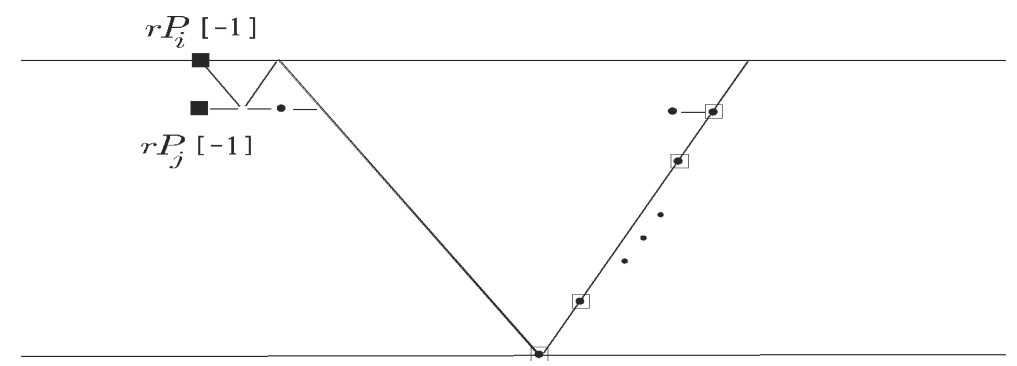

(b) $r P_{t}[-1]:$ In case $i \rightarrow t \leftarrow j$ is a subquiver of $Q_{\Lambda}$. 


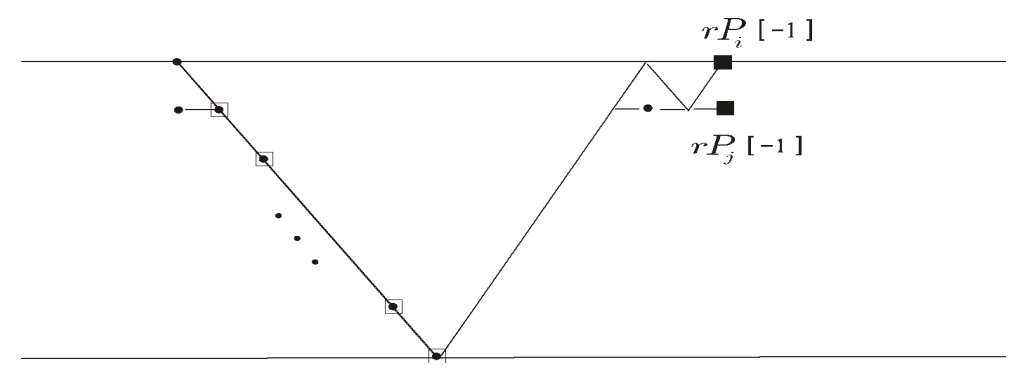

Proof. Let $i \leftarrow t \rightarrow j$ be a subquiver of $Q_{\Lambda}$ such that $r P_{i} / \operatorname{soc} P_{i} \simeq$ $r P_{j} / \operatorname{soc} P_{j}$ (the other case is dual). By 2.3 , we get an arrow $r P_{i}[-1] \rightarrow$ $r P_{j} / \operatorname{soc} P_{j}[0]$ in $\mathbb{Z} \mathbf{D}_{N}$ with $r P_{i}[-1]$ either in the middle or in the top border of $\mathbb{Z} \mathbf{D}_{N}$ since $N>4$. Then, applying 1.12 (a) to the arrows $t \rightarrow j$ and $t \rightarrow i$, we get the result.

Proposition 2.7. Let $\Lambda$ be a trivial extension of Cartan class $\mathbf{D}_{N}$ with $N>4$, and let $\Lambda$ be such that either $i \leftarrow t \rightarrow j$ or $i \rightarrow t \leftarrow j$ is a subquiver of $Q_{\Lambda}$.

(a) The following conditions are equivalent:

(a1) $r P_{i} / \operatorname{soc} P_{i} \simeq r P_{j} / \operatorname{soc} P_{j}$ and $r P_{t} / \operatorname{soc} P_{t}$ is indecomposable;

(a2) $r P_{t}[0]$ belongs to the bottom border of $\mathbb{Z} \mathbf{D}_{N}$.

(b) If $h_{\mathbb{Z} \mathbf{D}_{N}}\left(r P_{i}[0]\right)>N-2$ and $h_{\mathbb{Z} \mathbf{D}_{N}}\left(r P_{j}[0]\right)>N-2$, then

$$
h_{\mathbb{Z} \mathbf{D}_{N}}\left(r P_{t}[0]\right) \leq N-2 .
$$

Proof. Let ${ }_{S} \Gamma_{\Lambda}[0]$ be a lifting of ${ }_{S} \Gamma_{\Lambda}$ to $\mathbb{Z} \mathbf{D}_{N}$ at $r P_{t}$.

(a) The fact that (a1) implies (a2) follows easily from 2.6. Suppose that $r P_{t}[0]$ is in the bottom border of $\mathbb{Z} \mathbf{D}_{N}$ and assume that $i \leftarrow t \rightarrow j$ is a subquiver of $Q_{\Lambda}$ (the other case is dual). So, by 1.12 (a), we get that $\tau^{-1} r P_{i}[-1]$ and $\tau^{-1} r P_{j}[-1]$ are in $\operatorname{Supp}\left(-, r P_{t}[0]\right)$. Then $r P_{i}[-1]$ and $r P_{j}[-1]$ belong to $\operatorname{Supp}\left(-, \tau r P_{t}[0]\right)$. On the other hand, since $k\left(\mathbb{Z} \mathbf{D}_{N}\right)\left(r P_{i}[0], r P_{j}[0]\right)=0=k\left(\mathbb{Z} \mathbf{D}_{N}\right)\left(r P_{j}[0], r P_{i}[0]\right)$, we get by 1.11 (a) that the vertices $r P_{i}[-1]$ and $r P_{j}[-1]$ correspond to the vertices of $\operatorname{Supp}\left(-, \operatorname{\tau r} P_{t}[0]\right)$ indicated with $\square$ in the picture below. 


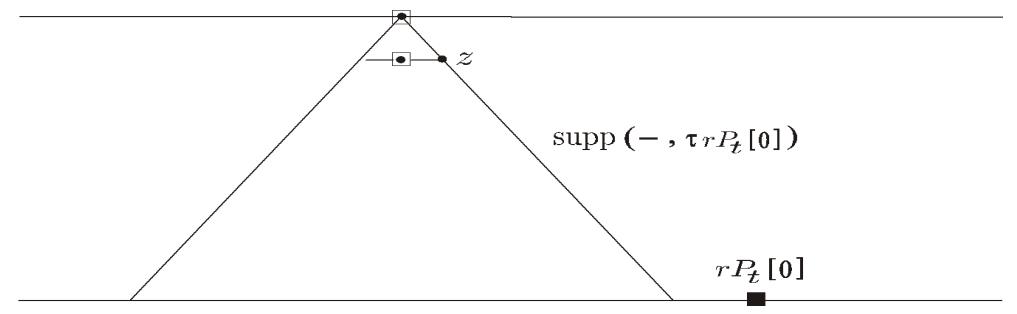

Hence $z=r P_{i} / \operatorname{soc} P_{i}[-1]=r P_{j} / \operatorname{soc} P_{j}[-1] ;$ and then

$$
r P_{i} / \operatorname{soc} P_{i} \simeq r P_{j} / \operatorname{soc} P_{j} \text {. }
$$

(b) Let $i \rightarrow t \leftarrow j$ be a subquiver of $Q_{\Lambda}$ (for the other case the proof is dual). Suppose that $r P_{t}[0]$ is in the top border of $\mathbb{Z} \mathbf{D}_{N}$. Then, by 1.12 (a), we get that $r P_{i}[0]$ and $r P_{j}[0]$ are in $\operatorname{Supp}\left(\tau^{-1} r P_{t}[0],-\right)$. The shaded region in the picture below corresponds to the vertices of $\operatorname{Supp}\left(\tau^{-1} r P_{t}[0],-\right)$.

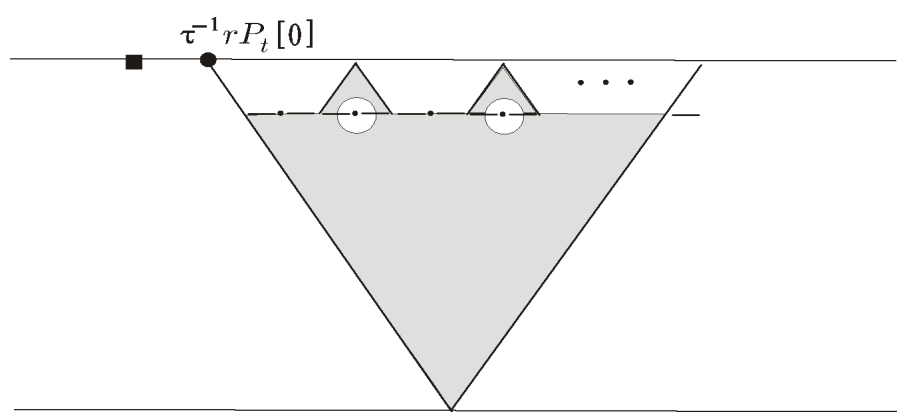

Since $h_{\mathbb{Z} \mathbf{D}_{N}}\left(r P_{i}[0]\right)>N-2$ and $h_{\mathbb{Z} \mathbf{D}_{N}}\left(r P_{j}[0]\right)>N-2$, we get that either $k\left(\mathbb{Z} \mathbf{D}_{N}\right)\left(r P_{i}[0], r P_{j}[0]\right) \neq 0$ or $k\left(\mathbb{Z D}_{N}\right)\left(r P_{j}[0], r P_{i}[0]\right) \neq 0$, which is a contradiction since $k\left(\mathbb{Z D}_{N}\right)\left(r P_{i}[0], r P_{j}[0]\right)$ and $k\left(\mathbb{Z} \mathbf{D}_{N}\right)\left(r P_{j}[0], r P_{i}[0]\right)$ are both zero because $i \neq j$. Therefore $r P_{t}[0]$ is not in the top border of $\mathbb{Z} \mathbf{D}_{N}$. Analogously, it can be proven that $r P_{t}[0]$ is not in the middle border of $\mathbb{Z} \mathbf{D}_{N}$.

\subsection{Lifting $r P_{j}$ to $\mathbb{Z} \mathbf{D}_{N}$ for some special vertices of $Q_{\Lambda}$.}


Proposition 2.8. Let $\Lambda$ be a trivial extension of Cartan class $\mathbf{D}_{N}$.

(a) Let $\gamma=z_{1} \rightarrow z_{2} \rightarrow \cdots \rightarrow z_{\ell-1} \rightarrow z_{\ell}$ be a path contained in a elemental cycle of $Q_{\Lambda}$ such that $P_{z_{\ell}} / \operatorname{soc} P_{z_{\ell}} \simeq r P_{z_{\ell-1}}, \cdots$, $P_{z_{2}} / \operatorname{soc} P_{z_{2}} \simeq r P_{z_{1}}$. Then, for any lifting of ${ }_{S} \Gamma_{\Lambda}$ to $\mathbb{Z} \mathbf{D}_{N}$ at $r P_{z_{\ell}}$, we have that $r P_{z_{\ell}}[0], r P_{z_{\ell-1}}[0], \cdots, r P_{z_{1}}[0]$ are consecutive vertices in one of the three borders of $\mathbb{Z} \mathbf{D}_{N}$; and moreover, this border is the bottom one if $\ell \geq 3$.

(b) If $\Lambda$ is of class I then $h_{\mathbb{Z} \mathbf{D}_{N}}\left(r P_{i}[0]\right)>N-2$ for $i=1,4$.

(c) Let $\Lambda$ be of class II and $i=1,2,3$. Then, we have that $r P_{i}[0]$ belongs to some border of $\mathbb{Z} \mathbf{D}_{N}$; and moreover, if $N>4$ and $\Lambda$ is either of class $I I_{2}$ or $I I_{3}$, then $h_{\mathbb{Z} \mathbf{D}_{N}}\left(r P_{i}[0]\right)>N-2$.

Proof. (a) Follows easily from 2.4.

(b) Since $\Lambda$ is of class $I$, by 2.2 (b), we get that $r P_{1} / \operatorname{soc} P_{1} \simeq$ $r P_{4} /$ soc $P_{4}$. Then, by 2.3 (b), we obtain the result.

(c) By 2.2 (c), we have that $r P_{i} / \operatorname{soc} P_{i}$ is indecomposable; and therefore, $r P_{i}[0]$ is in some border of $\mathbb{Z} \mathbf{D}_{N}$. Suppose that $N>4$ and $\Lambda$ is either of class $I I_{2}$ or $I I_{3}$. So, by $2.2(\mathrm{c} 2)$, we conclude that $r P_{c_{t}} / \operatorname{soc} P_{c_{t}} \not z r P_{3} / \operatorname{soc} P_{3}, r P_{b_{m}} / \operatorname{soc} P_{b_{m}} \not \subset r P_{2} / \operatorname{soc} P_{2}$, and $r P_{c_{1}} /$ soc $P_{c_{1}} \not r P_{3} / \operatorname{soc} P_{3}$. Therefore, applying 2.7 to the arrows $c_{t} \rightarrow$ $1 \leftarrow 3,2 \rightarrow 3 \leftarrow b_{m}, c_{1} \leftarrow 2 \rightarrow 3$ of $Q_{\Lambda}$, we get that $h_{\mathbb{Z} \mathbf{D}_{N}}\left(r P_{i}[0]\right)>$ $N-2$ since $r P_{i}[0]$ belongs to some border of $\mathbb{Z} \mathbf{D}_{N}$ for $i=1,2,3$.

Proposition 2.9. Let $\Lambda$ be a trivial extension of Cartan class $\mathbf{D}_{N}$ with $\Lambda$ of class $I I_{1}$ and $N>4$. Then, the following statements hold.

(a) $h_{\mathbb{Z} \mathbf{D}_{N}}\left(r P_{i}[0]\right)>N-2$ for $i=1,2,3$.

(b) For any lifting of ${ }_{S} \Gamma_{\Lambda}$ to $\mathbb{Z} \mathbf{D}_{N}$ at $r P_{3}$ such that $h_{\mathbb{Z} \mathbf{D}_{N}}\left(r P_{3}[0]\right)=$ $N-1$, we have that $r P_{1}, r P_{2}$ and $r P_{3}$ lift to $\mathbb{Z} \mathbf{D}_{N}$ as follows 


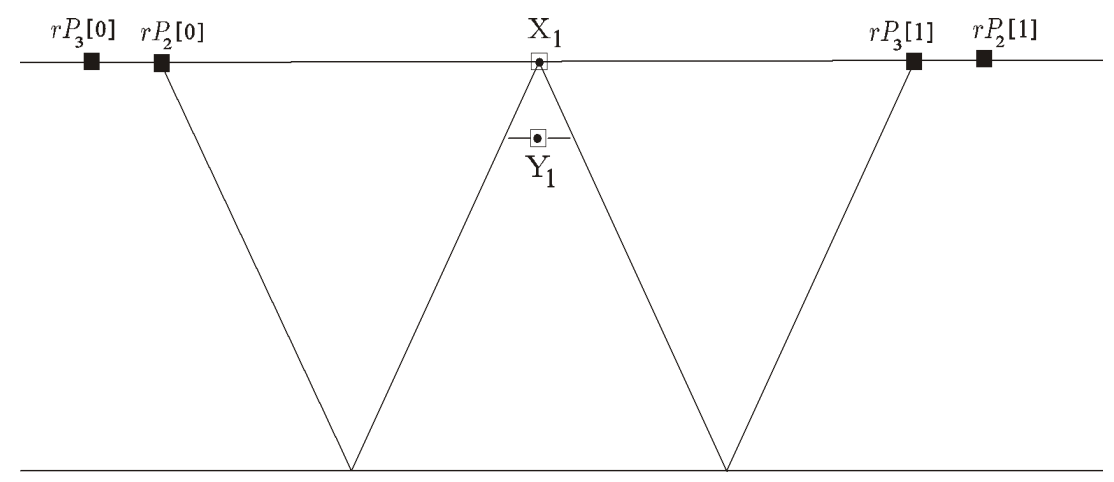

$$
\text { where } r P_{1}[0]= \begin{cases}X_{1} & \text { if } N \text { is odd } \\ Y_{1} & \text { if } N \text { is even. }\end{cases}
$$

Proof. By 2.2 (c1), it follows $r P_{1} / \operatorname{soc} P_{1} \not x r P_{a_{n}} / \operatorname{soc} P_{a_{n}}$ and $P_{3} /$ soc $P_{3} \simeq r P_{2}$. Hence, applying 2.7 to the subquiver $1 \rightarrow 2 \leftarrow a_{n}$ of $Q_{\Lambda}$, we get that $r P_{2}[0]$ is not in the bottom border of $\mathbb{Z} \mathbf{D}_{N}$. On the other hand, by $2.8(\mathrm{c})$, we know that $r P_{2}[0]$ is in some border of $\mathbb{Z} \mathbf{D}_{N}$. Hence, we conclude that $h_{\mathbb{Z} \mathbf{D}_{N}}\left(r P_{2}[0]\right)>N-2$.

Furthermore, since $\tau^{-1} r P_{3} \simeq r P_{2}$, we have that $r P_{3}$ and $r P_{2}$ lift consecutively to the same border of $\mathbb{Z} \mathbf{D}_{N}$. Thus, for a lifting of ${ }_{S} \Gamma_{\Lambda}$ to $\mathbb{Z} \mathbf{D}_{N}$ at $r P_{3}$ with $h_{\mathbb{Z} \mathbf{D}_{N}}\left(r P_{3}[0]\right)=N-1$, we get the picture given in 2.9 (b). We prove now that $r P_{1}[0]=X_{1}$ if $N$ is odd, otherwise $r P_{1}[0]=Y_{1}$.

Since $1 \rightarrow 2$ and $3 \rightarrow 1$ are arrows of $Q_{\Lambda}$, we conclude by 1.12 (a) that $r P_{1}[0] \in \operatorname{Supp}\left(\tau^{-1} r P_{2}[0],-\right)$ and $\tau^{-1} r P_{1}[0] \in \operatorname{Supp}\left(-, r P_{3}[1]\right)$; and so,

$$
r P_{1}[0] \in \operatorname{Supp}\left(\tau^{-1} r P_{2}[0],-\right) \cap \operatorname{Supp}\left(-, \tau r P_{3}[1]\right) .
$$

Furthermore, by $\left[8\right.$, Proposition 3.1] $\operatorname{Supp}(x,-)=\operatorname{Supp}\left(-, \nu_{\mathbf{D}_{N}}(x)\right)$ for any vertex $x$ of $\mathbb{Z} \mathbf{D}_{N}$. Thus

$$
r P_{1}[0] \in \operatorname{Supp}\left(-, \nu_{\mathbf{D}_{N}}\left(\tau^{-1} r P_{2}[0]\right) \cap \operatorname{Supp}\left(\nu_{\mathbf{D}_{N}}^{-1}\left(\tau r P_{3}[1]\right),-\right) .\right.
$$

Suppose that $N$ is even. Then, by 1.11 (b), the shaded region of the picture below correspond to the vertices of the set

$$
\operatorname{Supp}\left(\nu_{\mathbf{D}_{N}}^{-1}\left(\tau r P_{3}[1]\right),-\right) \cap \operatorname{Supp}\left(-, \nu_{\mathbf{D}_{N}}\left(\tau^{-1} r P_{2}[0]\right) .\right.
$$




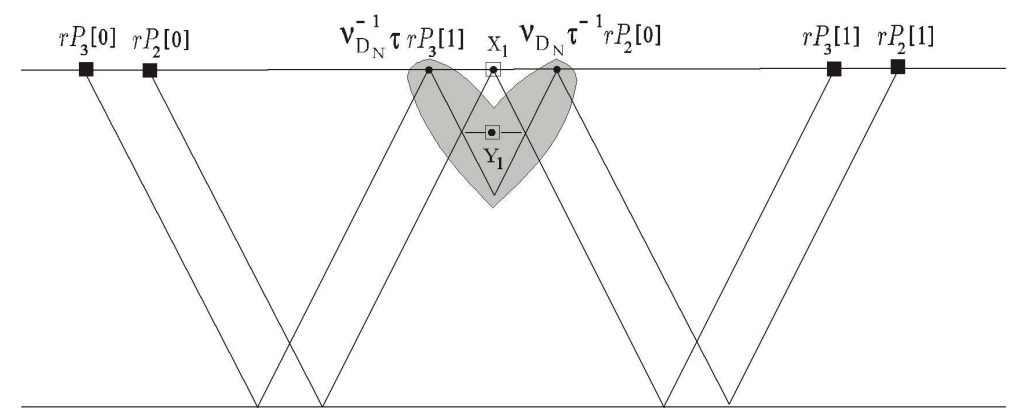

Since $r P_{1}[0]$ is in some border of $\mathbb{Z} \mathbf{D}_{N}$, we get that

$$
r P_{1}[0] \in\left\{\nu_{\mathbf{D}_{N}}^{-1}\left(\tau r P_{3}[1]\right), Y_{1}, \nu_{\mathbf{D}_{N}}\left(\tau^{-1} r P_{2}[0]\right)\right\} .
$$

Therefore, $r P_{1}[0]=Y_{1}$ since $k\left(\mathbb{Z} \mathbf{D}_{N}\right)\left(r P_{3}[0], \nu_{\mathbf{D}_{N}}^{-1}\left(\tau r P_{3}[1]\right)\right) \neq 0$ and $k\left(\mathbb{Z} \mathbf{D}_{N}\right)\left(\nu_{\mathbf{D}_{N}}\left(\tau^{-1} r P_{2}[0]\right), r P_{2}[1]\right) \neq 0$. Analogously, it can be proven that $r P_{1}[0]=X_{1}$ if $N$ is odd.

Proposition 2.10. Let $\Lambda$ be a trivial extension of Cartan class $\mathbf{D}_{N}$ with $\Lambda$ of class III. Then, the following statements hold.

(a) $h_{\mathbb{Z} \mathbf{D}_{N}}\left(r P_{1}[0]\right)=N-2$.

(b) For $i=2,3$, we have that $r P_{i}[0]$ belongs to some border of $\mathbb{Z} \mathbf{D}_{N} ;$ and moreover, $h_{\mathbb{Z} \mathbf{D}_{N}}\left(r P_{i}[0]\right)>N-2$ if $N>4$.

(c) For any lifting of ${ }_{S} \Gamma_{\Lambda}$ to $\mathbb{Z} \mathbf{D}_{N}$ at $r P_{1}$ such that $h_{\mathbb{Z} \mathbf{D}_{N}}\left(r P_{3}[0]\right)=$ $N-1$, we have that $r P_{1}, r P_{2}$ and $r P_{3}$ lift to $\mathbb{Z} \mathbf{D}_{N}$ as follows

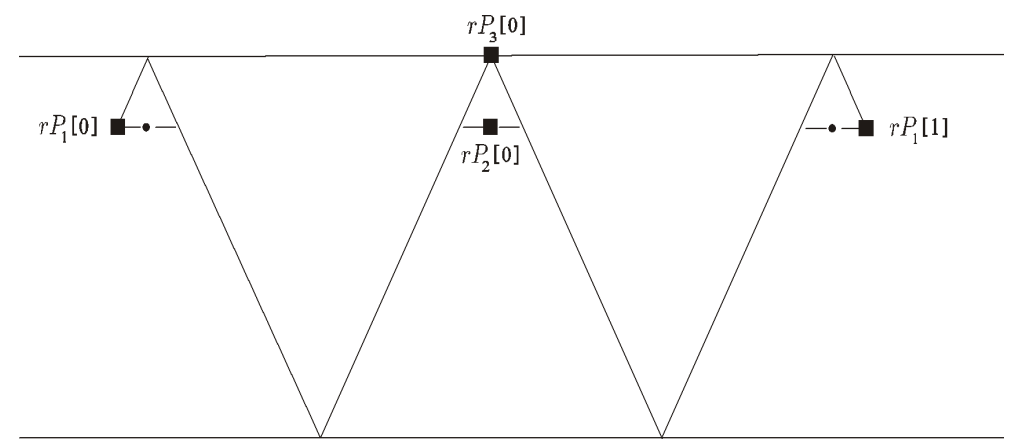


Proof. By $2.2(\mathrm{~d})$, we have that $r P_{1} /$ soc $P_{1}$ has three non-isomorphic indecomposable direct summands; so, we get that $h_{\mathbb{Z} \mathbf{D}_{N}}\left(r P_{1}[0]\right)=$ $N-2$. On the other hand, by 2.2 (d), we obtain that $r P_{2} / \operatorname{soc} P_{2} \simeq$ $r P_{3} /$ soc $P_{3}$. Therefore, by 2.3 (a), it follows that $r P_{i}[0]$ belongs to some

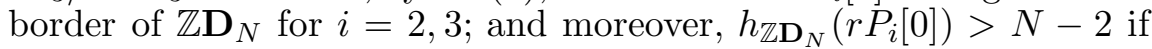
$N>4$.

Let $N>4$, and consider a lifting of ${ }_{S} \Gamma_{\Lambda}$ to $\mathbb{Z} \mathbf{D}_{N}$ at $r P_{1}$ with $h_{\mathbb{Z} \mathbf{D}_{N}}\left(r P_{3}[0]\right)=N-1$. Applying 1.12 to the arrows $2 \rightarrow 1$ and $3 \rightarrow 1$ of $Q_{\Lambda}$, we obtain that $r P_{i}[0] \in \operatorname{Supp}\left(\tau^{-1} r P_{1}[0],-\right) \backslash \operatorname{Supp}\left(r P_{1}[0],-\right)$ for $i=2,3$. In the picture below, we have indicated with a circle the elements of $\operatorname{Supp}\left(\tau^{-1} r P_{1}[0],-\right) \backslash \operatorname{Supp}\left(r P_{1}[0],-\right)$ which are in some border of $\mathbb{Z} \mathbf{D}_{N}$.

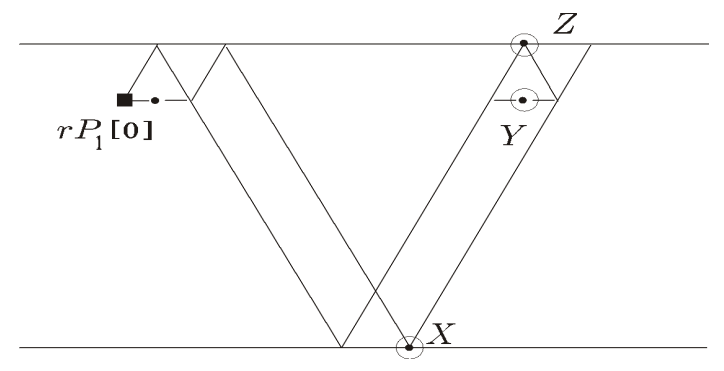

Since $h_{\mathbb{Z} \mathbf{D}_{N}}\left(r P_{i}[0]\right)>N-2$ for $i=2,3$, we have that $\left\{r P_{2}[0], r P_{3}[0]\right\}=$ $\{Y, Z\}$. Hence, using that $h_{\mathbb{Z} \mathbf{D}_{N}}\left(r P_{3}[0]\right)=N-1$, it follows that $Y=r P_{2}[0]$ and $Z=r P_{3}[0]$.

\subsection{The configurations for the case $\mathbf{D}_{4}$.}

In the following proposition, we study more closely the trivial extensions $\Lambda$ of Cartan class $\mathbf{D}_{4}$. In this case, we have that $\Lambda$ is either of class $I I_{1}$ or $I I I$. From 2.8 (c) and 2.10 (b), we know that $r P_{i}$ can be lifted to any border of $\mathbb{Z} \mathbf{D}_{4}$ for any vertex $i \in Q_{\Lambda}(i \neq 1$ if $\Lambda$ is of class $I I I)$. We choose the universal covering $\pi: \mathbb{Z}_{4} \rightarrow{ }_{S} \Gamma_{\Lambda}$ such that $h_{\mathbb{Z} \mathbf{D}_{4}}\left(r P_{t}[0]\right)>2$ for some fixed vertices $t=t_{1}, t_{2}$ of $Q_{\Lambda}$.

Proposition 2.11. Let $\Lambda$ be the trivial extension of Cartan class $\mathbf{D}_{4}$ given by one of the following quivers 


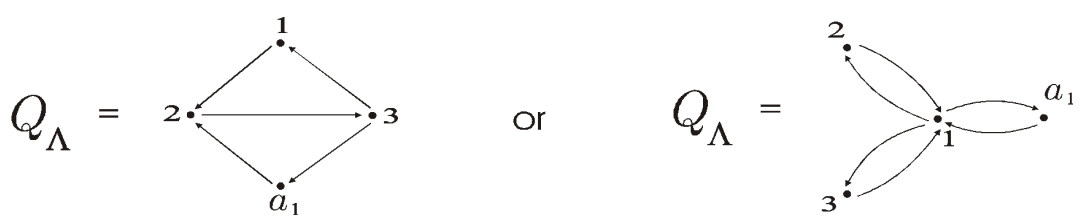

Let $\pi: \mathbb{Z} \mathbf{D}_{4} \rightarrow{ }_{S} \Gamma_{\Lambda}$ be the universal covering of ${ }_{S} \Gamma_{\Lambda}$ with $h_{\mathbb{Z} \mathbf{D}_{4}}\left(\pi^{-1}\left(r P_{3}\right)\right)$ $=3$ and $h_{\mathbb{Z} \mathbf{D}_{4}}\left(\pi^{-1}\left(r P_{a_{1}}\right)\right)=1$.

(a) If $\Lambda$ is of class $I I_{1}$ and ${ }_{S} \Gamma_{\Lambda}$ is lifted to $\mathbb{Z} \mathbf{D}_{4}$ at $r P_{3}$, then the configuration $\widetilde{\mathcal{C}_{\Lambda}}$ of $\mathbb{Z} \mathbf{D}_{4}$ associated to $\Lambda$ is

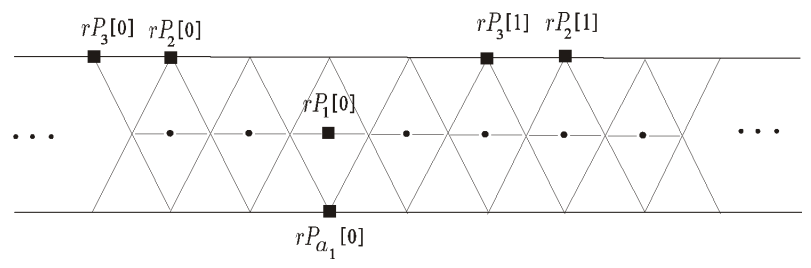

(b) If $\Lambda$ is of class III and ${ }_{S} \Gamma_{\Lambda}$ is lifted to $\mathbb{Z} \mathbf{D}_{4}$ at $r P_{1}$, then the configuration $\widetilde{\mathcal{C}_{\Lambda}}$ of $\mathbb{Z} \mathbf{D}_{4}$ associated to $\Lambda$ is

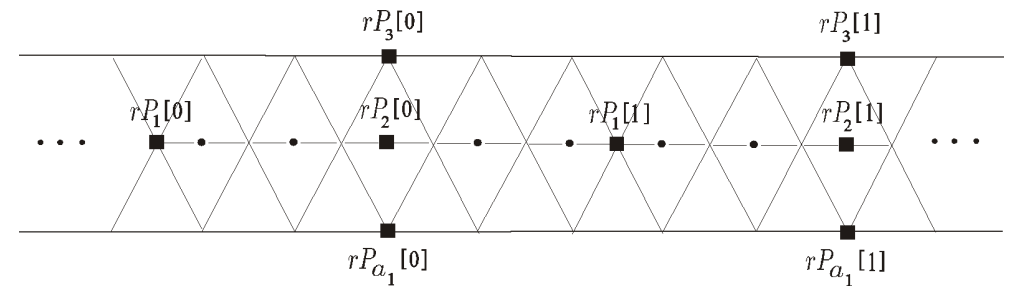

Proof. It is straightforward. 


\subsection{The configurations for $\Lambda$ minimal of type $\mathbf{D}_{N}$ with $N>4$.}

In what follows, we determine the vertices of $\mathbb{Z} \mathbf{D}_{N}$ corresponding to the configuration $\widetilde{\mathcal{C}_{\Lambda}}$ if $\Lambda$ is minimal and $N>4$.

Proposition 2.12. Let $\Lambda$ be a trivial extension of Class $I$ and Cartan class $\mathbf{D}_{N}$ with $N>4, \pi: \mathbb{Z} \mathbf{D}_{N} \rightarrow{ }_{S} \Gamma_{\Lambda}$ be the universal covering of ${ }_{S} \Gamma_{\Lambda}$ such that $h_{\mathbb{Z} \mathbf{D}_{N}}\left(\pi^{-1}\left(r P_{1}\right)\right)=N-1$; and take a lifting of ${ }_{S} \Gamma_{\Lambda}$ to $\mathbb{Z} \mathbf{D}_{N}$ at $\tau\left(r P_{1} / \operatorname{soc} P_{1}\right)$. Then $r P_{1}$ and $r P_{4}$ lift to $\mathbb{Z} \mathbf{D}_{N}$ as in the picture below; and furthermore, if $\Lambda$ is minimal (see the picture in 1.2), the configuration $\widetilde{\mathcal{C}_{\Lambda}}$ of $\mathbb{Z} \mathbf{D}_{N}$ associated to $\Lambda$ is as follows

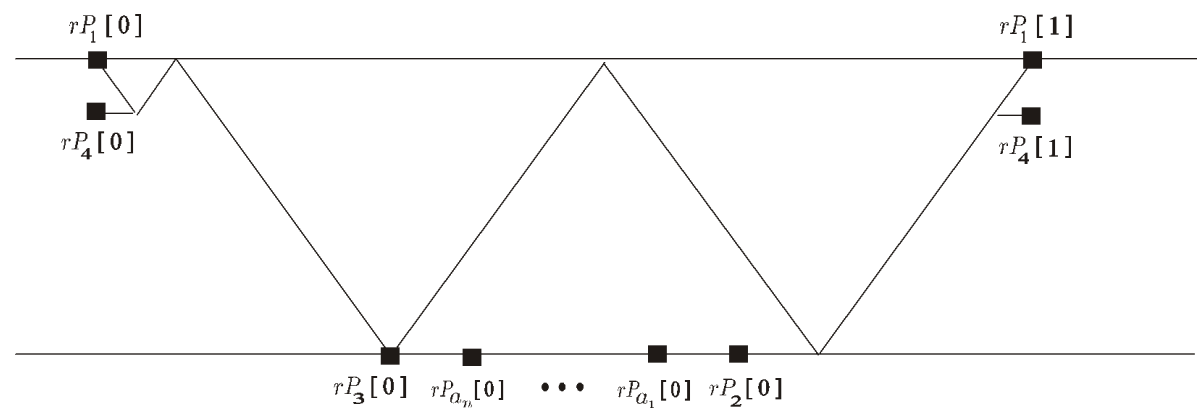

Proof. By 2.2 (b), we know that $r P_{1} / \operatorname{soc} P_{1} \simeq r P_{4} / \operatorname{soc} P_{4}$. Then, from 2.3 (a), we get that $r P_{1}[0]$ and $r P_{4}[0]$ are immediate predecessors of $r P_{1} / \operatorname{soc} P_{1}[0]$ and $h_{\mathbb{Z} \mathbf{D}_{N}}\left(r P_{i}[0]\right)>N-2$ for $i=1,4$ since $N>4$. Using that the universal covering $\pi: \mathbb{Z} \mathbf{D}_{N} \rightarrow{ }_{S} \Gamma_{\Lambda}$ we have chosen is such that $h_{\mathbb{Z} \mathbf{D}_{N}}\left(r P_{1}[0]\right)=N-1$, we conclude that $r P_{1}$ and $r P_{4}$ lift to $\mathbb{Z} \mathbf{D}_{N}$ as in the previous picture. On the other hand, by 1.5 and since $\Lambda$ is minimal of class $I$, it can be proven that $P_{3} / \operatorname{soc} P_{3} \simeq$ $r P_{a_{n}}, P_{a_{n}} / \operatorname{soc} P_{a_{n}} \simeq r P_{a_{n-1}}, \cdots, P_{a_{2}} / \operatorname{soc} P_{a_{2}} \simeq r P_{a_{1}}, P_{a_{1}} / \operatorname{soc} P_{a_{1}} \simeq$ $r P_{2}$. So, applying 2.8 (a) to the path $2 \rightarrow a_{1} \rightarrow a_{2} \rightarrow \cdots \rightarrow a_{n} \rightarrow 3$ in $Q_{\Lambda}$, we get that the radicals $r P_{3}, r P_{a_{n}}, \cdots, r P_{a_{1}}, r P_{2}$ lift consecutively to the bottom border of $\mathbb{Z} \mathbf{D}_{N}$. On the other hand $r P_{3}[0] \in$ $\operatorname{Supp}\left(\tau^{-1} r P_{1}[0],-\right)$ since $1 \leftarrow 3$ is an arrow in $Q_{\Lambda}$; and so, we get the previous picture, proving the result.

Let $\Lambda$ be a trivial extension of class $I I$. We recall (see 1.2, 1.3 and 1.9) that the class $I I$ is divided in 3 subclasses. Moreover, in case 
$N>4$, we have by 2.8 (c) and 2.9 (a) that $h_{\mathbb{Z} \mathbf{D}_{N}}\left(r P_{i}[0]\right)>N-2$ for the vertices $i=1,2,3$ of $Q_{\Lambda}$.

Lemma 2.13. Let $\Lambda$ be a trivial extension of Cartan class $\mathbf{D}_{N}$ with $N>4$ and $\Lambda$ minimal of class II. For $m>0$, we consider the cycle $B=3 \rightarrow 1 \rightarrow b_{1} \rightarrow b_{2} \rightarrow \cdots \rightarrow b_{m} \rightarrow 3$ of $Q_{\Lambda}$ and the universal covering $\pi: \mathbb{Z D}_{N} \rightarrow{ }_{S} \Gamma_{\Lambda}$ of ${ }_{S} \Gamma_{\Lambda}$ with $h_{\mathbb{Z} \mathbf{D}_{N}}\left(\pi^{-1}\left(r P_{3}\right)\right)=N-1$. Then, for a lifting of ${ }_{S} \Gamma_{\Lambda}$ to $\mathbb{Z} \mathbf{D}_{N}$ at $r P_{3}$, the radical of the projective associated to each vertex of the cycle $B$ lifts to $\mathbb{Z} \mathbf{D}_{N}$ as is indicated in the following picture

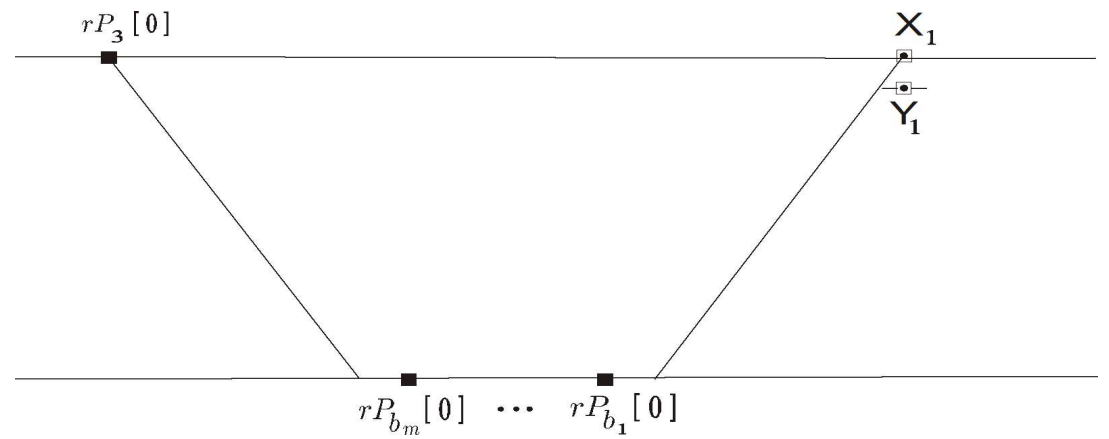

where $r P_{1}[0]= \begin{cases}X_{1} & \text { if } N-m-3 \text { is even, } \\ Y_{1} & \text { if } N-m-3 \text { is odd. }\end{cases}$

Proof. Assume that $m>0$, and let $\gamma$ be the path $b_{1} \rightarrow b_{2} \rightarrow \cdots \rightarrow$ $b_{m}$ in $Q_{\Lambda}$. Since $\Lambda$ is minimal of class $I I$, we get by 1.8 and 2.1 that $\gamma$ belongs to a single elemental cycle of $Q_{\Lambda}$ and that $b_{1}, b_{2}, \cdots, b_{m}$ are insertion vertices of $Q_{\Lambda}$. So we obtain from 2.2 (a) the following isomorphisms

$$
P_{b_{m}} / \operatorname{soc} P_{b_{m}} \simeq r P_{b_{m-1}}, \cdots, P_{b_{2}} / \operatorname{soc} P_{b_{2}} \simeq r P_{b_{1}} .
$$

Hence, by 2.8 (a), we have that $r P_{b_{m}}, r P_{b_{m-1}}, \cdots, r P_{b_{1}}$ lift consecutively to some border of $\mathbb{Z} \mathbf{D}_{N}$. We assert that this border is the bottom one. Indeed, suppose that $h_{\mathbb{Z} \mathbf{D}_{N}}\left(r P_{b_{m}}[0]\right)>N-2$. Applying 2.7 (b) to the subquiver $2 \rightarrow 3 \leftarrow b_{m}$ of $Q_{\Lambda}$, we obtain that $h_{\mathbb{Z} \mathbf{D}_{N}}\left(r P_{3}[0]\right) \leq N-2$; giving a contradiction. Thus $r P_{b_{m}}[0]$ is in the bottom border of $\mathbb{Z} \mathbf{D}_{N}$ and $\tau^{-i} r P_{b_{m}}[0]=r P_{b_{m-i}}[0]$ for $i=1,2, \cdots, m-1$. On the other hand, applying 1.12 (a) to the arrows $b_{m} \rightarrow 3,1 \rightarrow b_{1}$ of $Q_{\Lambda}$, we have that $r P_{b_{m}}[0] \in \operatorname{Supp}\left(\tau^{-1} r P_{3}[0],-\right)$ 
and $r P_{1}[0] \in \operatorname{Supp}\left(\tau^{-1} r P_{b_{1}}[0],-\right)$. So we get the previous picture and also that $r P_{1}[0] \in\left\{X_{1}, Y_{1}\right\}$ since $h_{\mathbb{Z} \mathbf{D}_{N}}\left(r P_{1}[0]\right)>N-2$. Now, we prove that $r P_{1}[0]$ is either $X_{1}$ or $Y_{1}$ depending on the parity of $N-m-3$.

Since $3 \rightarrow 1$ is an arrow in $Q_{\Lambda}$, we have from 1.2 and [8, Proposition 3.1 (a)] that

$$
r P_{1}[0] \in \operatorname{Supp}\left(-, \tau r P_{3}[1]\right)=\operatorname{Supp}\left(\nu_{\mathbf{D}_{N}}^{-1}\left(\tau r P_{3}[1]\right),-\right) .
$$

Suppose that $N$ is even (if $N$ is odd the proof is likewise). Consider the following picture.

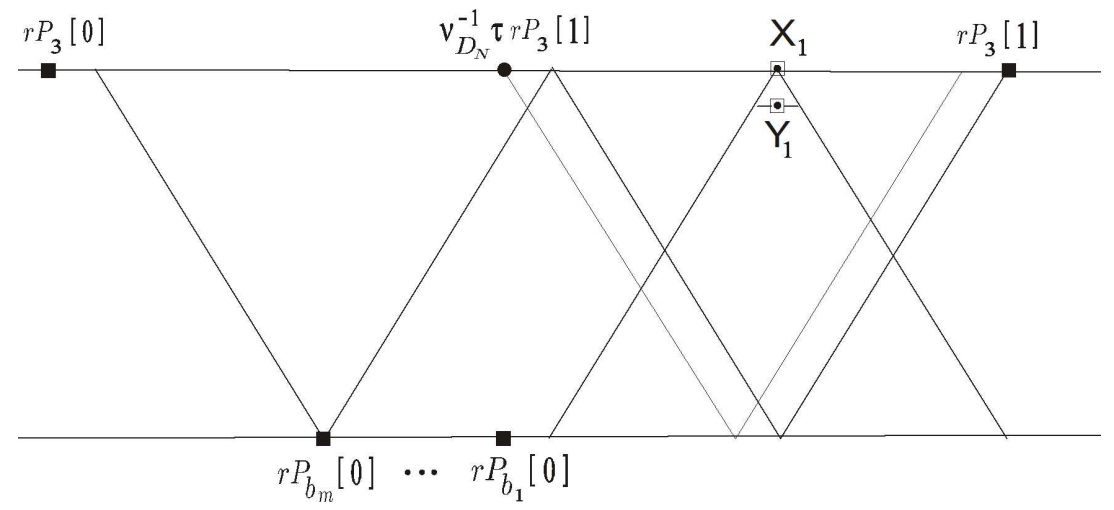

Since $r P_{1}[0] \in \operatorname{Supp}\left(\nu_{\mathbf{D}_{N}}^{-1}\left(\tau r P_{3}[1]\right),-\right) \cap\left\{X_{1}, Y_{1}\right\}$, we obtain from 1.11 (a) that

$$
r P_{1}[0]= \begin{cases}X_{1} & \text { if } m+1 \text { is even, } \\ Y_{1} & \text { si } m+1 \text { is odd. }\end{cases}
$$

Using that $N-m-3=N-(m+1)-2$ and $N$ is even, it follows that $N-m-3$ is even (resp. odd) if and only if $m+1$ is even (resp. odd); proving the result.

Proposition 2.14. Let $\Lambda$ be a minimal trivial extension of Class II and Cartan class $\mathbf{D}_{N}$ with $N>4$. Consider the universal covering $\pi: \mathbb{Z} \mathbf{D}_{N} \rightarrow{ }_{S} \Gamma_{\Lambda}$ of ${ }_{S} \Gamma_{\Lambda}$ such that $h_{\mathbb{Z} \mathbf{D}_{N}}\left(\pi^{-1}\left(r P_{3}\right)\right)=N-1$. Then, for a lifting of ${ }_{S} \Gamma_{\Lambda}$ to $\mathbb{Z D}_{N}$ at $r P_{3}$, the configuration $\widetilde{\mathcal{C}_{\Lambda}}$ associated to $\Lambda$ is as follows 


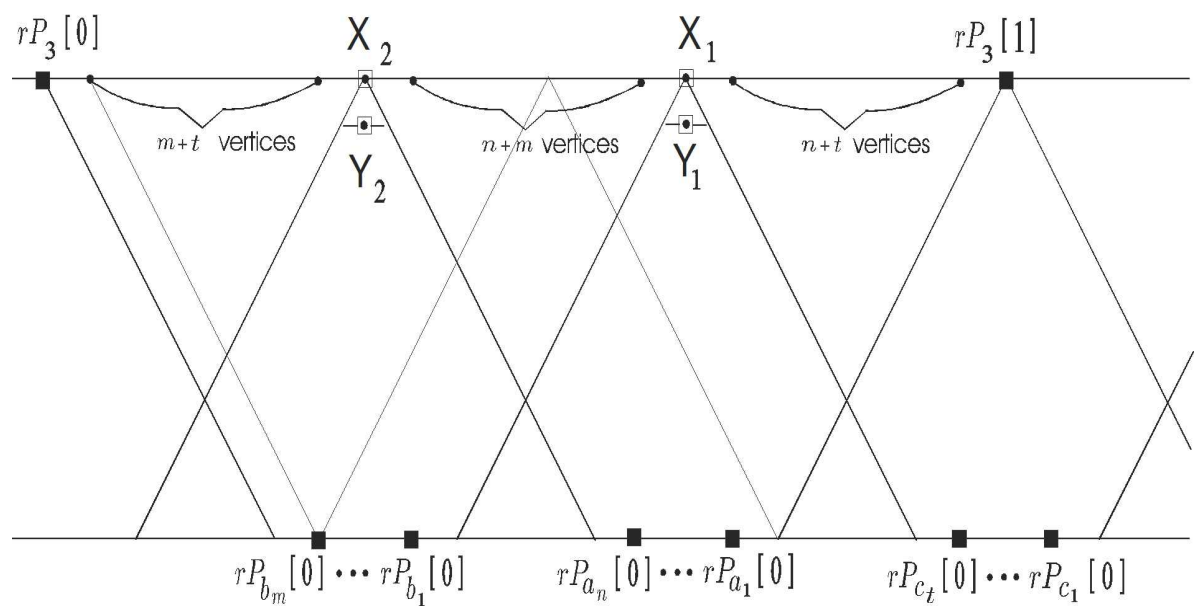

$$
\begin{aligned}
\text { where } r P_{1}[0] & =\left\{\begin{array}{ll}
X_{1} & \text { if } t+n \text { is even, } \\
Y_{1} & \text { if } t+n \text { is odd. }
\end{array}\right. \text { and } \\
r P_{2}[0] & = \begin{cases}X_{2} & \text { if } t+m \text { is even, } \\
Y_{2} & \text { if } t+m \text { is odd. }\end{cases}
\end{aligned}
$$

Proof. Suppose that $\Lambda$ is minimal of class $I I_{1}$. By 2.9 (b), we have that $r P_{2}$ and $r P_{3}$ lift to the same border of $\mathbb{Z} \mathbf{D}_{N}$. So, we can apply 2.13 to the cycle $A=2 \rightarrow 3 \rightarrow a_{1} \rightarrow \cdots \rightarrow a_{n} \rightarrow 2$ of $Q_{\Lambda}$; and so, from 2.9 , we get that the configuration $\widetilde{\mathcal{C}_{\Lambda}}$ associated to $\Lambda$ is as follows

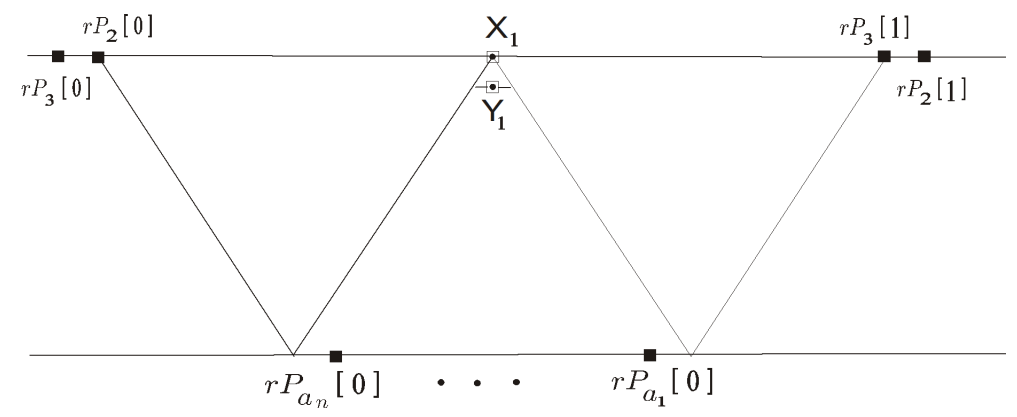

São Paulo J.Math.Sci. 4, 2 (2010), 273-349 
where $r P_{1}[0]= \begin{cases}X_{1} & \text { if } N \text { is odd, } \\ Y_{1} & \text { if } N \text { is even. }\end{cases}$

We know that $N=n+3$ since $\Lambda$ is minimal of class $I I_{1}$; and so, $N$ is odd if and only if $n$ is even, proving the result for $\Lambda$ of class $I I_{1}$.

Suppose that $\Lambda$ is either of class $I I_{2}$ or $I I_{3}$. Applying 1.12 (a) to the arrows $c_{t} \rightarrow 1 \rightarrow b_{1}$, we have that $r P_{1}[0] \in \operatorname{Supp}\left(\tau^{-1} r P_{b_{1}}[0],-\right)$ and $r P_{c_{t}}[0] \in \operatorname{Supp}\left(\tau^{-1} r P_{1}[0],-\right)$; and so, by applying 2.13 to the elemental cycles $1 \rightarrow 2 \rightarrow c_{1} \rightarrow c_{2} \rightarrow \cdots \rightarrow c_{t} \rightarrow 1$ and $3 \rightarrow 1 \rightarrow$ $b_{1} \rightarrow b_{2} \rightarrow \cdots \rightarrow b_{m} \rightarrow 3$ of $Q_{\Lambda}$, we obtain the following picture

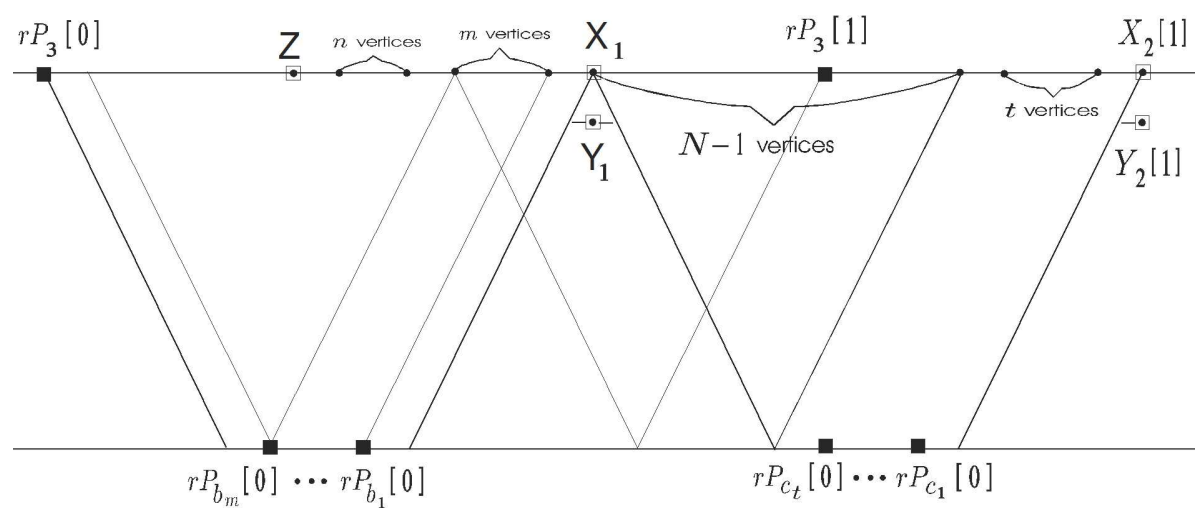

where $r P_{1}[0] \in\left\{X_{1}, Y_{1}\right\}$ since $h_{\mathbb{Z} \mathbf{D}_{N}}\left(r P_{1}[0]\right)>N-2$. Moreover, we have the arrow $2 \rightarrow c_{1}$ in $Q_{\Lambda}$; and therefore, by 1.12, we obtain that $r P_{2}[1] \in\left\{X_{2}[1], Y_{2}[1]\right\}$ since $h_{\mathbb{Z} \mathbf{D}_{N}}\left(r P_{2}[1]\right)>N-2$.

We assert that $Z=X_{2}$ for the $Z$ in the above picture. Indeed, since $\Lambda$ is minimal, we get $N=n+m+t+3$. Thus $1+n+m+N-1+t=2 N-3$ and hence $z=\tau^{1+n+m+N-1+t} X_{2}[1]=\tau^{2 N-3} X_{2}[1]=X_{2}$.

We assert that $r P_{2}[0]$ and $r P_{1}[0]$ satisfy the stated equalities. For $r P_{1}[0]$, it can be obtained from 2.13 using that $N=n+m+t+3$.

For $r P_{2}[0]$, we apply 1.12 (a) to the arrow $2 \rightarrow 3$ in $Q_{\Lambda}$ obtaining that $r P_{2}[1] \in \operatorname{Supp}\left(\tau^{-1} r P_{3}[1],-\right)$. Furthermore, from the above picture, it can be seen that $\tau^{-(m+t+1)} r P_{3}[1]=X_{2}[1]$. Then, by $1.11(\mathrm{~b})$, we conclude that $r P_{2}[1]=X_{2}[1]$ if $t+m$ is even, and $r P_{2}[1]=Y_{2}[1]$ if $t+m$ is odd. 
Proposition 2.15. Let $\Lambda$ be a minimal trivial extension of Class III (see 1.2) and Cartan class $\mathbf{D}_{N}$ with $N>4$. Consider the universal covering $\pi: \mathbb{Z D}_{N} \rightarrow{ }_{S} \Gamma_{\Lambda}$ of ${ }_{S} \Gamma_{\Lambda}$ with $h_{\mathbb{Z} \mathbf{D}_{N}}\left(\pi^{-1}\left(r P_{3}\right)\right)=N-$ 1. Then, for a lifting of ${ }_{S} \Gamma_{\Lambda}$ to $\mathbb{Z} \mathbf{D}_{N}$ at $r P_{1}$, the configuration $\widetilde{\mathcal{C}_{\Lambda}}$ associated to $\Lambda$ is as follows

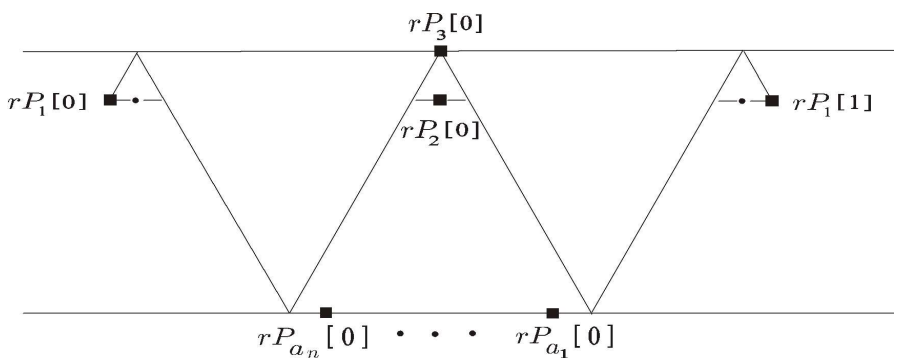

Proof. The radicals $r P_{1}, r P_{2}$ and $r P_{3}$ lift to $\mathbb{Z} \mathbf{D}_{N}$ as in 2.10. We prove that $r P_{a_{n}}, \cdots, r P_{a_{1}}$ lift consecutively to the bottom border of $\mathbb{Z} \mathbf{D}_{N}$ as in the preceding picture. Applying 1.12 (a) to the arrows $2 \rightarrow$ $1,3 \rightarrow 1$ and $a_{n} \rightarrow 1$ of $Q_{\Lambda}$, we have $r P_{i}[0] \in \operatorname{Supp}\left(\tau^{-1} r P_{1}[0],-\right) \backslash$ $\operatorname{Supp}\left(r P_{1}[0],-\right)$ for $i=2,3, a_{n}$. In the picture given in the proof of 2.10, we indicated with a circle the elements $X, Y$ and $Z$ of $\operatorname{Supp}\left(\tau^{-1} r P_{1}[0],-\right) \backslash \operatorname{Supp}\left(r P_{1}[0],-\right)$ which are in some border of $\mathbb{Z} \mathbf{D}_{N}$.

Consider the path $\gamma=a_{1} \rightarrow a_{2} \rightarrow \cdots \rightarrow a_{n}$ of $Q_{\Lambda}$. Since $\Lambda$ is minimal of class III, we obtain that $\gamma$ belongs to a elemental cycle of $Q_{\Lambda}$ and also that $a_{1}, a_{2}, \cdots, a_{n}$ are insertion vertices (see 1.8 and 2.1) in $Q_{\Lambda}$. Hence, by 2.2 (a), we get that $P_{a_{n}} / \operatorname{soc} P_{a_{n}} \simeq$ $r P_{a_{n-1}}, \cdots, P_{a_{2}} / \operatorname{soc} P_{a_{2}} \simeq r P_{a_{1}}$. Then, by 2.8 (a), the radicals $r P_{a_{n}}$, $r P_{a_{n-1}}, \cdots, r P_{a_{1}}$ lift consecutively to some border of $\mathbb{Z} \mathbf{D}_{N}$. Thus, it is enough to prove that $X=r P_{a_{n}}[0]$ (see the picture given in the proof of 2.10). By 2.10, we know that $Y=r P_{3}[0]$ and $Z=r P_{2}[0]$; therefore $X=r P_{a_{n}}[0]$, proving the result.

\subsection{Lifting $r P_{j}$ to $\mathbb{Z} \mathbf{D}_{N}$ for the vertices $j$ belonging to an elimination cycle of $Q_{\Lambda}$.}

In order to find, in the general case, the configuration $\widetilde{\mathcal{C}_{\Lambda}}$ associated to a given trivial extension $\Lambda$, we need firstly to know how can be lifted to $\mathbb{Z} \mathbf{D}_{N}$ the radicals of the projective modules associated with 
the vertices of an elimination cycle of $Q_{\Lambda}$ (see 2.1). So, the following result will be very useful throughout this paper.

Theorem 2.16. Let $\Lambda$ be a trivial extension of Cartan class $\mathbf{D}_{N}$ with $N>4$, and $C=z \leftarrow z_{1} \leftarrow z_{2} \leftarrow \cdots \leftarrow z_{m-1} \leftarrow z$ an elimination cycle of $Q_{\Lambda}$. Then, for a lifting ${ }_{S} \Gamma_{\Lambda}[0]$ of ${ }_{S} \Gamma_{\Lambda}$ to $\mathbb{Z D}_{N}$ at $r P_{z_{1}}$, we have that

(a) $h_{\mathbb{Z} \mathbf{D}_{N}}\left(r P_{z_{1}}[0]\right)=1$ and $\tau^{-i} r P_{z_{1}}[0]=r P_{z_{i+1}}[0]$ for $1 \leq i \leq$ $m-2$,

(b) $\operatorname{Supp}\left(\tau^{-1} r P_{z_{m-1}}[0],-\right) \cap \operatorname{Supp}\left(-, \tau r P_{z_{1}}[1]\right)=\left\{r P_{z}[0]\right\}$, and

(c) $h_{\mathbb{Z} \mathbf{D}_{N}}\left(r P_{z}[0]\right)=m$.

Proof. (a) Consider the path $\gamma:=z_{m-1} \rightarrow z_{m-2} \rightarrow \cdots \rightarrow z_{1}$ in $Q_{\Lambda}$. Then $\gamma$ belongs to the elemental cycle $C$ in $Q_{\Lambda}$. Therefore $z_{1}, z_{2}, \cdots, z_{m-1}$ are insertion vertices of $Q_{\Lambda}$. Hence, by 2.2 (a), we get the isomorphisms $P_{z_{1}} / \operatorname{soc} P_{z_{1}} \simeq r P_{z_{2}}, \cdots, P_{z_{m-2}} / \operatorname{soc} P_{z_{m-2}} \simeq r P_{z_{m-1}}$. Thus, by 2.8 (a), it follows that $\tau^{-i} r P_{z_{1}}[0]=r P_{z_{i+1}}[0]$ for $1 \leq i \leq$ $m-2$ and $r P_{z_{1}}[0]$ belongs to some border of $\mathbb{Z} \mathbf{D}_{N}$; furthermore, this border is the bottom one if $m \geq 4$. So, we have to analyze the cases $m=2$ and $m=3$.

Suppose that $m=2$. Then $C=z \leftarrow z_{1} \leftarrow z$; and hence by 1.12 (a) we get that $r P_{z}[0] \in \operatorname{Supp}\left(\tau^{-1} r P_{z_{1}}[0],-\right) \cap \operatorname{Supp}\left(-, \tau r P_{z_{1}}[1]\right)$. If $h_{\mathbb{Z} \mathbf{D}_{N}}\left(r P_{z_{1}}[0]\right)>N-2$. Then, by using 1.11 , it is not difficult to see that $h_{\mathbb{Z} \mathbf{D}_{N}}\left(r P_{z}[0]\right)=N-2$ and $\operatorname{Supp}\left(\tau^{-1} r P_{z_{1}}[0],-\right) \cap \operatorname{Supp}\left(-, \tau r P_{z_{1}}[1]\right)$ $=\left\{r P_{z}[0]\right\}$. In the following picture, we illustrate this fact if $h_{\mathbb{Z} \mathbf{D}_{N}}\left(r P_{z_{1}}[0]\right)=N-1$ and $N$ is even.

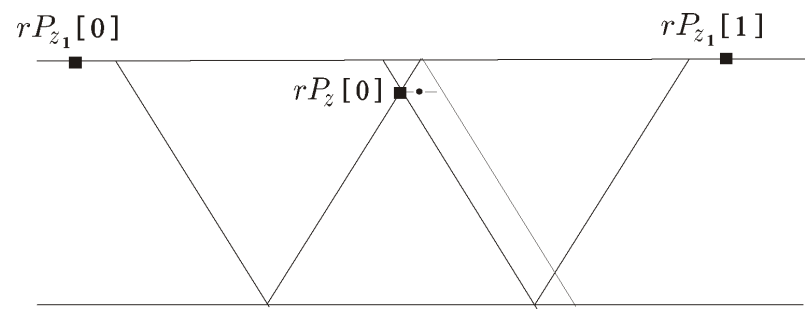

The equality $h_{\mathbb{Z} \mathbf{D}_{N}}\left(r P_{z}[0]\right)=N-2$ implies that $r P_{z} / \operatorname{soc} P_{z}$ has three indecomposable non-isomorphic direct summands. Using, on one hand, the combinatorial description ( see [6, Corollary 4.12]) of 
the number of the indecomposable non-isomorphic direct summands of $r P_{j} / \operatorname{soc} P_{j}$ for a vertex $j$ in $Q_{\Lambda}$; and on the other hand, the description of $\Lambda$ given in 1.8, it can be proven that $\Lambda$ is of class $I I I$ and $z=1$. Moreover, $\Lambda$ is minimal since $C$ is an elimination cycle of $Q_{\Lambda}$. Furthermore, since $N>4$, we get that $C=1 \leftarrow a_{n} \leftarrow \cdots \leftarrow a_{1} \leftarrow 1$, contradicting that $C$ is an elimination cycle of $Q_{\Lambda}$ (see 1.8). Hence, for $m=2$, we have that $r P_{z_{1}}[0]$ belongs to the bottom border of $\mathbb{Z} \mathbf{D}_{N}$.

Assume that $m=3$. Then $C=z \leftarrow z_{1} \leftarrow z_{2} \leftarrow z$; and so $P_{z_{1}} / \operatorname{soc} P_{z_{1}} \simeq r P_{z_{2}}$. Suppose that $h_{\mathbb{Z} \mathbf{D}_{N}}\left(r P_{z_{1}}[0]\right)>N-2$. Since $\tau^{-1} r P_{z_{1}}[0]=r P_{z_{2}}[0]$, it can be proven by using 1.11 that there is only one vertex $x \in{ }_{S} \Gamma_{\Lambda}[0]$ such that $h_{\mathbb{Z} \mathbf{D}_{N}}(x)>N-2$ and $x \notin$ $\operatorname{Supp}\left(r P_{z_{i}}[0],-\right) \cup \operatorname{Supp}\left(-, r P_{z_{i}}[1]\right)$ for $i=1,2$. In the following picture, we illustrate this fact in case $h_{\mathbb{Z} \mathbf{D}_{N}}\left(r P_{z_{1}}[0]\right)=N-1$, where $x=X_{1}$ if $N$ is odd and $x=X_{2}$ if $N$ is even.

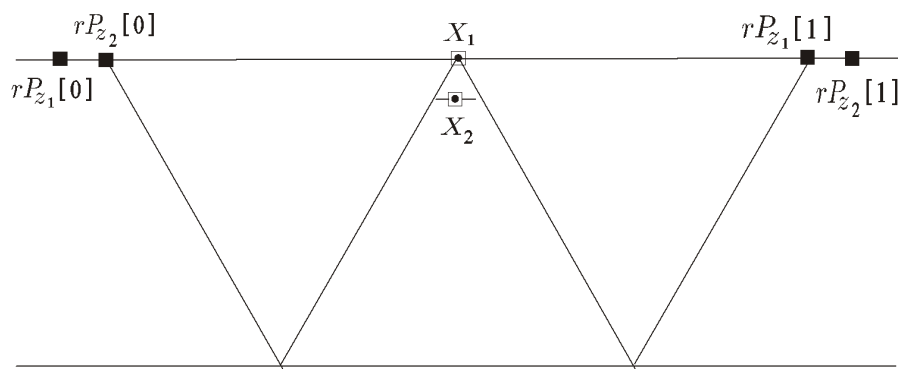

On the other hand, since $C$ is an elimination cycle of $Q_{\Lambda}$, we conclude from $2.8(\mathrm{~b})$ and (c), 2.9 (a) and 2.10 (b), that there are at least two vertices $i, j \in Q_{\Lambda}$ such that $i, j \notin\left\{z_{1}, z_{2}\right\}$ and $h_{\mathbb{Z} \mathbf{D}_{N}}\left(r P_{t}[0]\right)>N-2$ for $t=i, j$. Then we get two vertices $r P_{i}[0]$ and $r P_{j}[0]$ in ${ }_{S} \Gamma_{\Lambda}[0]$ satisfying the same property as the vertex $x$, contradicting the fact that there exists just one. Therefore, for $m=3$, we also obtain that $r P_{z_{1}}[0]$ belongs to the bottom border of $\mathbb{Z} \mathbf{D}_{N}$.

Finally, the proof of (b) and (c) follows easily from (a), 1.11 (a) and 1.12 (a).

Remark 2.17. Let $\Lambda$ and $C=z \leftarrow z_{1} \leftarrow z_{2} \leftarrow \cdots \leftarrow z_{m-1} \leftarrow z$ be as in 2.16. Then the radicals $r P_{z_{1}}, r P_{z_{2}}, \cdots, r P_{z_{m-1}}, r P_{z}$ lift to $\mathbb{Z} \mathbf{D}_{N}$ as in the following picture 


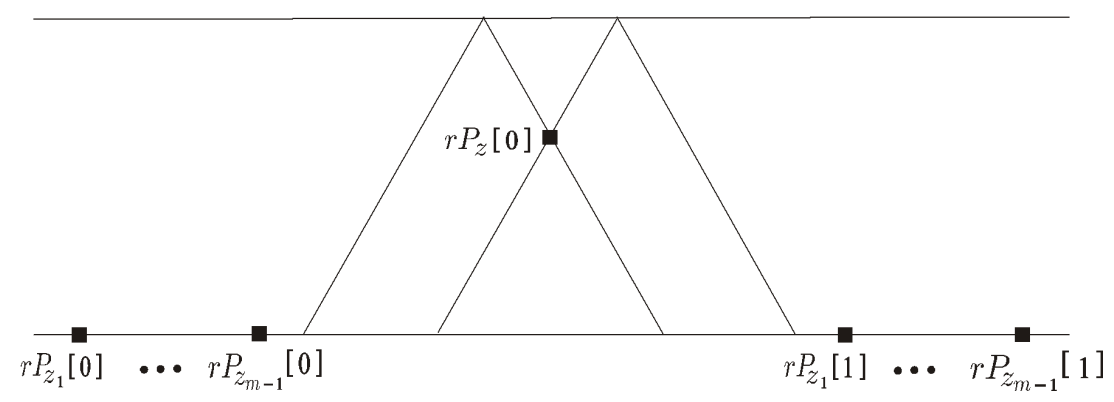

\section{The embedding of ${ }_{S} \Gamma_{\operatorname{End}_{\Lambda}(P)^{o p}}$ in ${ }_{S} \Gamma_{\Lambda}$}

For a given artin algebra $\Lambda$, we will use freely properties of the module category $\bmod \Lambda$ of finitely generated left $\Lambda$-modules, the stable category $\bmod \Lambda$ module projectives and the Auslander-Reiten translations $\tau$ and $\tau^{-1}$ as is found in [3].

Let $\Gamma$ be a trivial extension of Cartan class $\mathbf{D}_{N}, z$ an insertion vertex of $Q_{\Gamma}$ (see 2.1) and $\Lambda$ be the trivial extension of Cartan class $\mathbf{D}_{N+m-1}$ obtained from $\Gamma$ by inserting the cycle $C_{z}=z \leftarrow z_{1} \leftarrow z_{2} \leftarrow$ $\cdots \leftarrow z_{m-1} \leftarrow z$ at $z$. We will embed ${ }_{S} \Gamma_{\Gamma}$ into ${ }_{S} \Gamma_{\Lambda}$. This will allows us to deduce properties of ${ }_{S} \Gamma_{\Lambda}$ from properties of ${ }_{S} \Gamma_{\Gamma}$. Since $Q_{\Gamma}$ has less cycles than $Q_{\Lambda}$, we will use this embedding to reduce our problem to the minimal case, studied in the previous section.

We recall that $\Gamma \simeq \operatorname{End}_{\Lambda}(P)^{o p}$ where $P:=\coprod_{i \in\left(Q_{\Gamma}\right)_{0}}{ }_{\Lambda} P_{i}$. Consider (see $\left[9\right.$, Section 2]) the full subcategory $\mathcal{C}_{P}$ of $\bmod \Lambda$ whose objects are the modules $X$ such that the projective cover $P_{0}(X)$ of $X$ and the injective envelope $I_{0}(X)$ of $X$ belong to add $P$. Since $\Lambda$ is a weaklysymmetric algebra, from [9, Section 2], we have that the evaluation functor $e_{P}=\operatorname{Hom}_{\Lambda}(P,-): \bmod \Lambda \rightarrow \bmod \Gamma$, allowing the identification of $\bmod \Gamma$ with the full subcategory $\mathcal{C}_{P}$ of $\bmod \Lambda$, induces an equivalence $\underline{e}_{P}: \underline{\mathcal{C}_{P}} \rightarrow \underline{\bmod } \Gamma$ of stable categories. Let $\imath: \underline{\bmod } \Gamma \rightarrow \underline{\bmod } \Lambda$ be the full and faithful functor obtained by the composition of the inverse equivalence of $\underline{e}_{P}: \underline{\mathcal{C}_{P}} \rightarrow \underline{\bmod } \Gamma$ and the inclusion $\underline{\mathcal{C}_{P}} \subseteq \underline{\bmod } \Lambda$. In this section, we will study the behavior of the irreducible morphisms of $\underline{\bmod } \Gamma$ inside $\underline{\bmod } \Lambda$ using the embedding $\imath: \underline{\bmod } \Gamma \rightarrow \underline{\bmod } \Lambda$. 
Proposition 3.1. Let $\Gamma$ be a trivial extension of Cartan class $\mathbf{D}_{N}$, $z$ an insertion vertex of $Q_{\Gamma}$ and $\Lambda$ be the trivial extension obtained from $\Gamma$ by inserting the cycle $C_{z}=z \leftarrow z_{1} \leftarrow z_{2} \leftarrow \cdots \leftarrow z_{m-1} \leftarrow z$ at $z$. Consider the projective $\Lambda$-module $P=\coprod_{i \in\left(Q_{\Gamma}\right)_{0}{ }_{\Lambda} P_{i}}$ and the equivalence of stable categories $\underline{e}_{P}: \underline{\mathcal{C}}_{P} \rightarrow \underline{\bmod } \Gamma$. Then, the following statements hold.

(a) ind $\mathcal{C}_{P}$ decomposes in four connected components $\mathrm{X}^{-}, \mathrm{X}^{+}, \mathrm{Y}^{-}$ and $\overline{\mathrm{Y}^{+}}$which are convex subquivers of ${ }_{S} \Gamma_{\Lambda}$ as in the picture below

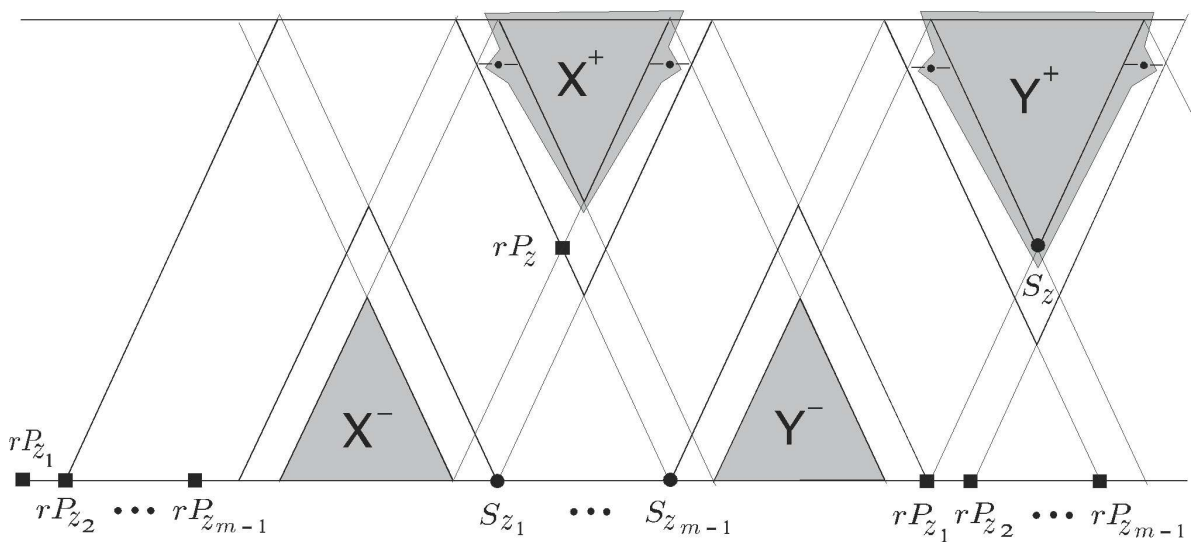

(b) For any $X, Y \in \operatorname{ind} \underline{\mathcal{C}_{P}}$ the following conditions are equivalent.

(b1) $f: X \rightarrow Y$ is irreducible in $\underline{\bmod } \Lambda$.

(b2) $X$ and $Y$ are in the same connected component of ind $\mathcal{C}_{P}$ in ${ }_{S} \Gamma_{\Lambda}$ and $\underline{e}_{P}(f): \underline{e}_{P}(X) \rightarrow \underline{e}_{P}(Y)$ is irreducible $\overline{\text { in }}$ $\underline{\bmod \Gamma}$.

(c) If $\chi$ is one of the quivers $\mathrm{X}^{ \pm}, \mathrm{Y}^{ \pm}$, then $\underline{e}_{P}(\chi)$ is a convex subquiver of ${ }_{S} \Gamma_{\operatorname{End}_{\Lambda}(P)^{o p}}$ and $\left.\underline{e}_{P}\right|_{\chi}: \chi \rightarrow \underline{e}_{P}(\chi)$ is an isomorphism of quivers.

Proof. (a) Let $Q$ be the projective $\Lambda$-module $\coprod_{i=1}^{m-1}{ }_{\Lambda} P_{z_{i}}$. Then, by $[9$, Theorem 2.4], we know that the objects of $\underline{\bmod } \Lambda$ which do not belong to $\underline{\mathcal{C}}_{P}$ coincides with $\operatorname{Supp} \underline{\operatorname{Hom}}_{\Lambda}(Q / \operatorname{soc} Q,-) \cup \operatorname{Supp} \underline{\operatorname{Hom}}_{\Lambda}(-, r Q)$. 
Thus, for any lifting ${ }_{S} \Gamma_{\Lambda}[0]$ of ${ }_{S} \Gamma_{\Lambda}$ to $\mathbb{Z} \mathbf{D}_{N}$ at $r P_{z_{1}}$, we get the equality

$$
{ }_{S} \Gamma_{\Lambda}[0] \backslash \operatorname{ind} \underline{\mathcal{C}_{P}}[0]=\cup_{i=1}^{m-1} \operatorname{Supp}\left(\tau^{-1} r P_{z_{i}}[0],-\right) \cup \cup_{i=1}^{m-1} \operatorname{Supp}\left(-, r P_{z_{i}}[0]\right) \text {. }
$$

On the other hand, by 2.17 , we know that the radicals $r P_{z_{1}}, \cdots, r P_{z_{m-1}}$ lift consecutively to the bottom border of $\mathbb{Z} \mathbf{D}_{N}$; furthermore, by using the shape (see in 1.11) that the supports of the functors $k\left(\mathbb{Z} \mathbf{D}_{N}\right)(x,-)$ and $k\left(\mathbb{Z} \mathbf{D}_{N}\right)(-, x)$ have in $\mathbb{Z} \mathbf{D}_{N}$, we obtain that the set

$$
\left.\cup_{i=1}^{m-1} \operatorname{Supp}\left(\tau^{-1} r P_{z_{i}}[0],-\right) \cup \cup_{i=1}^{m-1} \operatorname{Supp}\left(-, r P_{z_{i}}[0]\right)\right)
$$

corresponds to the shaded region of the picture below.

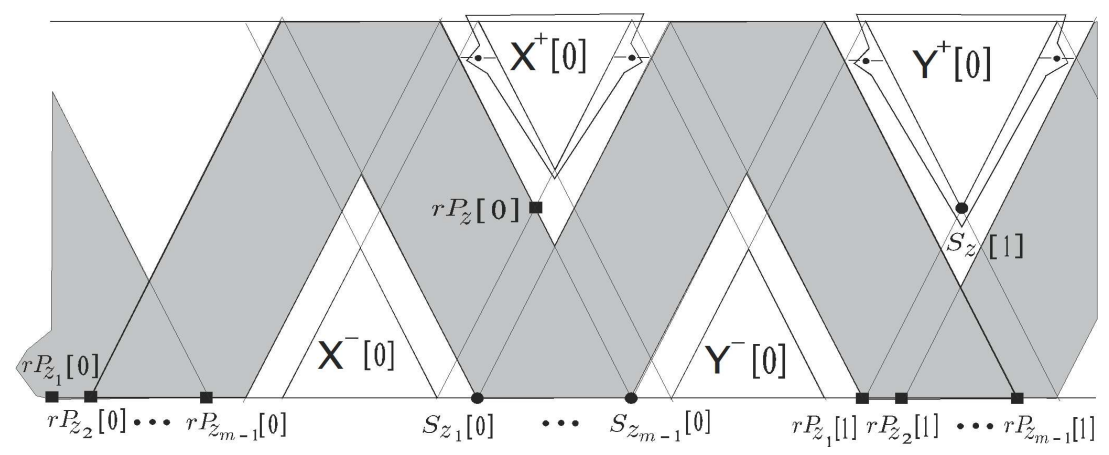

The lifting of the simple $\Lambda$-module $S_{i}$ for $i=z, z_{1}, \cdots, z_{m-1}$ was carried out using that $S_{i}[0]=\nu_{\mathbb{Z} \mathbf{D}_{N}}\left(\tau^{-1} r P_{i}[0]\right.$ ) (see [9, Proposition 3.3]); proving (a).

(b) Let $X, Y \in \operatorname{ind} \underline{\mathcal{C}_{P}}$.

(b1) $\Rightarrow$ (b2) Assume that $f: X \rightarrow Y$ is irreducible in $\underline{\bmod } \Lambda$. It follows directly, from [9, Proposition 2.9 (a)], that $\underline{e}_{P}(f): \underline{e_{P}}(X) \rightarrow$ $\underline{e}_{P}(Y)$ is irreducible in $\underline{\bmod } \Gamma$. On the other hand, there is an arrow $X \rightarrow Y$ in ${ }_{S} \Gamma_{\Lambda}$ with $X \in \chi$, where $\chi$ is one of the quivers $\mathrm{X}^{ \pm}, \mathrm{Y}^{ \pm}$. Suppose that $X$ and $Y$ are not in the same component of ind $\mathcal{C}_{P}$ in ${ }_{S} \Gamma_{\Lambda}$. Then, any path in ${ }_{S} \Gamma_{\Lambda}$ starting at $X$ and ending at $Y$ has length at least two, contradicting that $X \rightarrow Y$ is an arrow of ${ }_{S} \Gamma_{\Lambda}$; and so, we get that $X$ and $Y$ are in $\chi$. 
(b2) $\Rightarrow$ (b1) Assume that $X$ and $Y$ belong to $\chi$, where $\chi$ is one of the components $\mathrm{X}^{ \pm}, \mathrm{Y}^{ \pm}$, and also that $\underline{e}_{P}(f): \underline{e}_{P}(X) \rightarrow \underline{e}_{P}(Y)$ is irreducible in $\bmod \Gamma$. Suppose that $f: \bar{X} \rightarrow Y$ is not irreducible in $\underline{\bmod } \Lambda$. Then, there is a path in ${ }_{S} \Gamma_{\Lambda}$ induced by irreducible morphisms

$$
X=M_{0} \stackrel{f_{1}}{\rightarrow} M_{1} \stackrel{f_{2}}{\rightarrow} M_{2} \rightarrow \cdots \rightarrow M_{r-1} \stackrel{f_{r}}{\rightarrow} M_{r}=Y
$$

having non zero composition in $\underline{\bmod } \Lambda$. Hence, by [9, Proposition 2.9 (c)], it follows that $M_{j} \notin \underline{\mathcal{C}_{P}}$ for $j=1,2, \cdots, r-1$. This is a contradiction, since by (a) we know that $\chi$ is a convex subquiver of ${ }_{S} \Gamma_{\Lambda}$.

(c) It follows easily from (a) and (b).

Definition 3.2. Let $\Lambda$ be a trivial extension of Dynkin Cartan class. For $X \in{ }_{S} \Gamma_{\Lambda}$, we set:

- $\underline{\alpha}_{\Lambda}(X)$ is the number of arrows in ${ }_{S} \Gamma_{\Lambda}$ starting at $X$;

- $\overrightarrow{\mathcal{S}}_{X}\left(\right.$ resp. $\left.{ }_{X} \overrightarrow{\mathcal{S}}\right)$ is the section of ${ }_{S} \Gamma_{\Lambda}$ starting (resp. ending) at $X$

- $X^{+}:=\left\{Y \in \underline{\text { ind } \Lambda}\right.$ : there exists an arrow $X \rightarrow Y$ in $\left.{ }_{S} \Gamma_{\Lambda}\right\}$.

Corollary 3.3. With the hypothesis in 3.1, let $X \in \chi$ be such that $X \neq{ }_{\Lambda} S_{z}$, where $\chi$ is one of the components $\mathrm{X}^{ \pm}, \mathrm{Y}^{ \pm}$of ind $\underline{\mathcal{C}_{P}}$ in ${ }_{S} \Gamma_{\Lambda}$. If $X^{+} \not \subset \chi$ then

(a) there exists a sectional path in ${ }_{S} \Gamma_{\Lambda}$ (denoted also as $X \rightsquigarrow Y$ )

$$
X \stackrel{f_{1}}{\rightarrow} M_{1} \stackrel{f_{2}}{\rightarrow} M_{2} \rightarrow \cdots \rightarrow M_{m-1} \stackrel{f_{m}}{\rightarrow} Y
$$

such that $Y \in$ ind $\mathcal{C}_{P}, Y \notin \chi$ and $M_{\ell} \notin$ ind $\mathcal{C}_{P}$ for $\ell=$ $1,2, \cdots m-1$

(b) the morphism $\underline{e}_{P}(f): \underline{e}_{P}(X) \rightarrow \underline{e}_{P}(Y)$ is irreducible in $\underline{\bmod } \Gamma$, where $f:=f_{m} \cdots f_{2} f_{1}$.

Proof. (a) follows easily from 3.1; and (b) from [9, Proposition $2.9(\mathrm{~b})]$.

Theorem 3.4. With the hypothesis in 3.1, the following statements hold.

(a) For any $X$ and $Y$ in ind $\mathcal{C}_{P}$, which do not belong to the same component of ind $\underline{\mathcal{C}_{P}}$ in ${ }_{S} \overline{\Gamma_{\Lambda}}$, the following conditions are equivalent: 
(a1) there exists an irreducible morphism $\underline{e}_{P}(X) \rightarrow \underline{e}_{P}(Y)$ in $\underline{\bmod } \Gamma$;

(a2) there exists a sectional path $X \stackrel{f_{1}}{\rightarrow} M_{1} \stackrel{f_{2}}{\rightarrow} M_{2} \rightarrow \cdots \rightarrow$ $M_{r-1} \stackrel{f_{r}}{\longrightarrow} Y$ in ${ }_{S} \Gamma_{\Lambda}$ such that $M_{i} \notin \operatorname{ind} \underline{\mathcal{C}_{P}}$ for $i=1,2, \cdots$, $r-1$.

Moreover, if (a2) holds then, for $f:=f_{r} f_{r-1} \cdots f_{1}$, we have that $\underline{e}_{P}(f): \underline{e}_{P}(X) \rightarrow \underline{e}_{P}(Y)$ is irreducible in $\underline{\bmod } \Gamma$.

(b) For any $X \in \operatorname{ind} \underline{\mathcal{C}_{P}}$, we have that

$\underline{\alpha}_{\Gamma}\left(\underline{e}_{P}(X)\right)= \begin{cases}\underline{\alpha}_{\Lambda}(X) & \text { if } X \text { is not isomorphic to }{ }_{\Lambda} S_{z}, X_{1} \text { or } X_{2}, \\ \underline{\alpha}_{\Lambda}(X)-1 & \text { if } X \simeq{ }_{\Lambda}, \\ \underline{\alpha}_{\Lambda}(X)+1 & \text { if } X \simeq X_{1} \text { or } X \simeq X_{2},\end{cases}$

where $X_{1}:=\overrightarrow{\mathcal{S}}_{\tau^{-2} r_{\Lambda} P_{z_{m-1}}} \cap{ }_{\tau_{\Lambda} S_{z_{1}}} \overrightarrow{\mathcal{S}}$ and $X_{2}:=\overrightarrow{\mathcal{S}}_{\tau^{-1}{ }_{\Lambda} S_{z_{m-1}}} \cap$ $\tau r_{\Lambda} P_{z_{1}} \overrightarrow{\mathcal{S}}$.

(c) $\tau^{-1}\left(\underline{e}_{P}\left({ }_{\Lambda} S_{z}\right)\right) \simeq \underline{e}_{P}\left(\tau^{-2} r_{\Lambda} P_{z_{m-1}}\right)$.

Proof. By 3.1 we know that ind $\underline{\mathcal{C}_{P}}$ decomposes in four connected components: $\mathrm{X}^{-}, \mathrm{X}^{+}, \mathrm{Y}^{-}$and $\mathrm{Y}^{+}$. Moreover, $\underline{e}_{P}\left(\mathrm{X}^{ \pm}\right)$and $\underline{e}_{P}\left(\mathrm{Y}^{ \pm}\right)$are

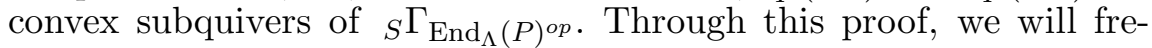
quently use the picture appearing in 3.1 ; and also, from this picture we see that the vertex $X_{1}$ (resp. $X_{2}$ ) corresponds to the top of the shaded triangle $\mathrm{X}^{-}$(resp. $\left.\mathrm{Y}^{-}\right)$.

Let $X \in \operatorname{ind} \underline{\mathcal{C}_{P}}$. Then $X$ belongs to $\chi$, where $\chi$ is one of the quivers $\mathrm{X}^{ \pm}, \mathrm{Y}^{ \pm}$. Since $\Lambda$ is of Cartan class $\mathbf{D}_{N+m-1}$, we get that $1 \leq \underline{\alpha}_{\Lambda}(X) \leq$ 3. If $X^{+} \subseteq \chi$ then the result is obtained as a direct consequence of 3.1 .

Assume that $X^{+} \nsubseteq \chi$. If $X \neq{ }_{\Lambda} S_{z}$ then, by 3.3, there is a sectional path $X \rightsquigarrow Z$ in ${ }_{S} \Gamma_{\Lambda}$ such that $Z \in$ ind $\underline{\mathcal{C}_{P}}, Z \notin \chi$ and $X \rightsquigarrow Z$ induces an irreducible morphism $\underline{e}_{P}(X) \rightarrow \underline{\underline{e}_{P}}(Z)$ in $\underline{\bmod } \Gamma$. On the other hand, if $X^{+} \cap \chi \neq \emptyset$ then there is an arrow $X \rightarrow Y^{\prime}$ in $\chi$ and this arrow induces, by 3.1, an irreducible morphism $\underline{e}_{P}(X) \rightarrow \underline{e}_{P}\left(Y^{\prime}\right)$ in $\bmod \Gamma$. Hence, we have two manners of constructing irreducible morphisms in $\underline{\bmod } \Gamma:(i)$ by using an arrow $X \rightarrow Y^{\prime}$ of $\chi$ and (ii) by using a sectional path $X \rightsquigarrow Z$ as above. Then, to prove (a), it is enough to see that the irreducible morphisms in $\underline{\bmod } \Gamma$, starting at $\underline{e}_{P}(X)$, are obtained either by $(i)$ or $(i i)$.

For any $W \in \chi$ with $W^{+} \nsubseteq \chi$, we denote by $\mathcal{A}_{1}(W)$ the set of arrows 
$W \rightarrow Y^{\prime}$ of $\chi$ and by $\mathcal{A}_{>1}(W)$ the set of sectional paths $W \rightsquigarrow Z$ in ${ }_{S} \Gamma_{\Lambda}$ (as in $\left.(i i)\right)$ such that $Z \in$ ind $\underline{\mathcal{C}_{P}}$ and $Z \notin \chi$.

We will prove the result by considering the cardinal of the set $\mathcal{A}_{1}(X) \cup$ $\mathcal{A}_{>1}(X)$. If $\operatorname{card}\left(\mathcal{A}_{1}(X) \cup \mathcal{A}_{>1}(X)\right)=2$ or $\operatorname{card}\left(\mathcal{A}_{1}(X) \cup \mathcal{A}_{>1}(X)\right)=3$, the proof is straightforward.

Let $\operatorname{card}\left(\mathcal{A}_{1}(X) \cup \mathcal{A}_{>1}(X)\right)=1$. So $\operatorname{card}\left(\mathcal{A}_{1}(X)\right)=1$ or $\operatorname{card}\left(\mathcal{A}_{>1}(X)\right)=1$. In case $\operatorname{card}\left(\mathcal{A}_{>1}(X)\right)=1$ the proof is also straightforward.

Suppose that $\operatorname{card}\left(\mathcal{A}_{1}(X)\right)=1$. Then $X={ }_{\Lambda} S_{z} \in \mathrm{Y}^{+}$since we have an arrow $X \rightarrow Y_{1}$ in $\mathrm{Y}^{+}$and there are no sectional paths $X \rightsquigarrow Y$ as in 3.3. Let $W \in$ ind $\mathcal{C}_{P}$, we assert that if there exists an irreducible morphism $\underline{e}_{P}(X) \rightarrow \underline{e}_{P}(W)$ then $W \simeq Y_{1}$. Indeed, let $Q$ be the projective $\Lambda$-module $\coprod_{j=1}^{m-1}{ }_{\Lambda} P_{z_{i}}$ and $X \rightarrow Y_{1} \coprod Y_{2}$ be an irreducible morphism in $\underline{\bmod } \Lambda$. Consider the section $\overrightarrow{\mathcal{S}}_{X}$ starting at $X$. Then $Y_{1}, Y_{2} \in \overrightarrow{\mathcal{S}}_{X}$ and therefore

$$
\operatorname{Supp} \underline{\operatorname{Hom}}_{\Lambda}\left(Y_{2},-\right) \subseteq \bigcup_{i=1}^{m-1} \operatorname{Supp} \underline{\operatorname{Hom}} \Lambda\left(\tau^{-1} r P_{z_{i}},-\right)=\operatorname{Supp} \underline{\operatorname{Hom}}_{\Lambda}(M,-)
$$

where $M:=Q / \operatorname{soc} Q$, as can be deduced from the picture below

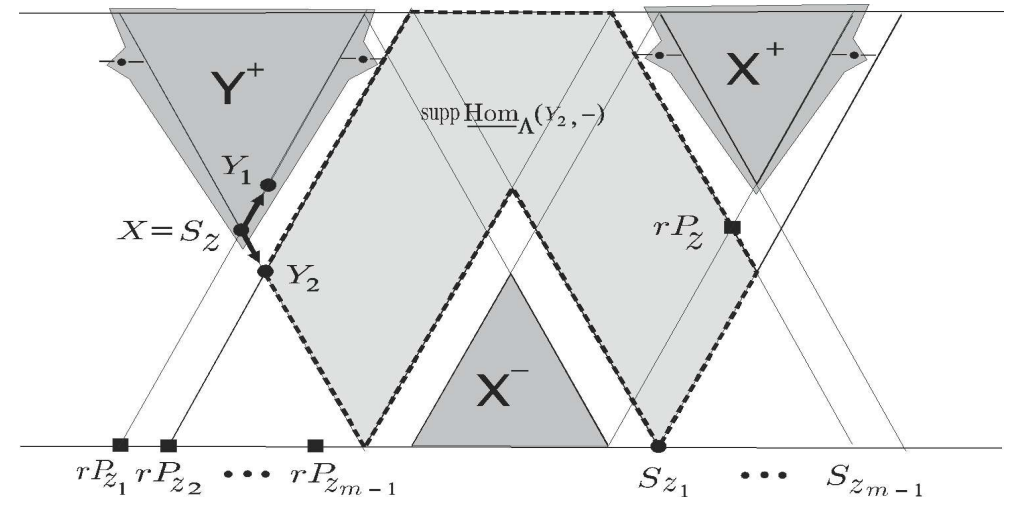

Let $\underline{e}_{P}(\varphi): \underline{e}_{P}(X) \rightarrow \underline{e}_{P}(W)$ be irreducible. Then $\varphi=f_{1}+f_{2}$ where $f_{i}$, for $i=1,2$, is the composition of a chain $X \rightarrow Y_{i} \rightarrow$ . $\rightarrow \cdots \rightarrow W$ of irreducible morphisms in ind $\Lambda$. If $f_{2}$ is non zero then $W \in \operatorname{Supp} \underline{\operatorname{Hom}}_{\Lambda}\left(Y_{2},-\right) \subseteq \operatorname{Supp} \underline{\operatorname{Hom}}_{\Lambda}(Q / \operatorname{soc} Q,-)$; giving a contradiction since $W \in$ ind $\underline{\mathcal{C}_{P}}$ implies, from [9, Theorem 2.4], that 
$\underline{\operatorname{Hom}}_{\Lambda}(Q / \operatorname{soc} Q, W)=0$. Thus $\varphi=f_{1}$ and therefore $\underline{e}_{P}(W) \simeq \underline{e}_{P}\left(Y_{1}\right)$ since $\underline{e}_{P}(X) \rightarrow \underline{e}_{P}\left(Y_{1}\right)$ is irreducible. Using the fidelity of the functor $\underline{e}_{P}$, we get that $W \simeq Y_{1}$. Hence, we proved that the unique arrow in ${ }_{S} \Gamma_{\Gamma}$ starting at $\underline{e}_{P}(X)$ is $\underline{e}_{P}(X) \rightarrow \underline{e}_{P}\left(Y_{1}\right)$. Hence $\underline{\alpha}_{\Gamma}\left(\underline{e}_{P}(X)\right)=1=$ $\underline{\alpha}_{\Lambda}(X)-1$.

To prove (c), we use the sectional path $h: Y_{1} \rightsquigarrow \tau^{-2} r_{\Lambda} P_{z_{m-1}}$ (see the picture above). Since $\underline{e}_{P}(h): \underline{e}_{P}\left(Y_{1}\right) \rightarrow \underline{e}_{P}\left(\tau^{-2} r_{\Lambda} P_{z_{m-1}}\right)$ is irreducible and $\underline{\alpha}_{\Gamma}\left(\underline{e}_{P}(X)\right)=1$, we conclude that $\tau^{-1}\left(\underline{e}_{P}\left({ }_{\Lambda} S_{z}\right)\right) \simeq$ $\underline{e}_{P}\left(\tau^{-2} r_{\Lambda} P_{z_{m-1}}\right)$; proving the result.

Theorem 3.5. Let $\Gamma$ be a trivial extension of Cartan class $\mathbf{D}_{N}$ with $N>4$. If $z$ is an insertion vertex of $Q_{\Gamma}$, then $r_{\Gamma} P_{z}[0]$ and ${ }_{\Gamma} S_{z}[0]$ belong to the bottom border of $\mathbb{Z} \mathbf{D}_{N}$.

Proof. Let $\Lambda$ be the trivial extension obtained from $\Gamma$ by inserting the cycle $C_{z}=z \leftarrow z_{1} \leftarrow z$ at $z$. Consider the projective $\Lambda$-module $P=\coprod_{i \in\left(Q_{\Gamma}\right)_{0} \Lambda} P_{i}$ and the equivalence $\underline{e}_{P}: \underline{\mathcal{C}_{P}} \rightarrow \underline{\bmod } \Gamma$ of stable categories. Since, by $\left[9\right.$, Proposition 3.3], $r_{\Gamma} P_{z}[0]=\tau \nu_{\mathbf{D}_{N}}^{-1}\left({ }_{\Gamma} S_{z}[0]\right)$ and the Nakayama permutation $\nu_{\mathbf{D}_{N}}$ sends the bottom border of $\mathbb{Z} \mathbf{D}_{N}$ to itself (see 1.11), it is enough to prove that ${ }_{\Gamma} S_{z}[0]$ belongs to the bottom border of $\mathbb{Z} \mathbf{D}_{N}$. Indeed, by 3.1 , we know that ind $\mathcal{C}_{P}$ splits inside ${ }_{S} \Gamma_{\Lambda}$ as four connected components. In the picture below, we have drawn in ${ }_{S} \Gamma_{\Lambda}$ three of these components and the position of the $\Lambda$-modules $r P_{t}$ and $S_{t}$ for $t=z, z_{1}$.

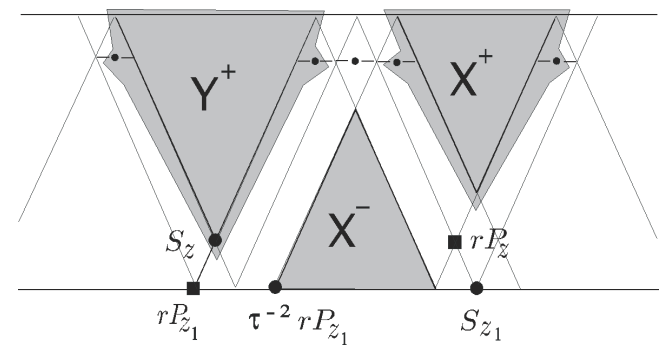

Moreover, by 3.4 (c), we know that $\tau^{-1}\left(\underline{e}_{P}\left({ }_{\Lambda} S_{z}\right)\right) \simeq \underline{e}_{P}\left(\tau^{-2} r_{\Lambda} P_{z_{1}}\right)$; and since $\tau^{-2} r_{\Lambda} P_{z_{1}}[0]$ is in the bottom border of $\mathbb{Z} \mathbf{D}_{N+1}$ and $N>$ 4 , we get that the vertex $\underline{e}_{P}\left(\tau^{-2} r_{\Lambda} P_{z_{1}}\right)[0]$ is so. Hence $\underline{e}_{P}\left({ }_{\Lambda} S_{z}\right)[0]$ belongs to the bottom border of $\mathbb{Z} \mathbf{D}_{N}$. On the other hand, by $[9$, 
Theorem 2.8], we have that $\underline{e}_{P}\left({ }_{\Lambda} S_{z}\right) \simeq{ }_{\Gamma} S_{z}$; and therefore ${ }_{\Gamma} S_{z}[0]$ is in the bottom border of $\mathbb{Z} \mathbf{D}_{N}$.

Corollary 3.6. Let $\Gamma$ be a trivial extension of Cartan class $\mathbf{D}_{N}$, $z$ an insertion vertex of $Q_{\Gamma}$ and $\Lambda$ the trivial extension obtained from $\Gamma$ by inserting the cycle $C_{z}=z \leftarrow z_{1} \leftarrow z_{2} \leftarrow \cdots \leftarrow z_{m-1} \leftarrow z$ at $z$. Then, for the projective $\Lambda$-module $P:=\coprod_{i \in\left(Q_{\Gamma}\right)_{0}{ }_{\Lambda} P_{i} \text { and the equivalence }}$ $\underline{e}_{P}: \mathcal{C}_{P} \rightarrow \underline{\bmod } \Gamma$ of stable categories, we have that: $(a) \tau_{\Lambda} S_{z_{1}} \in \mathcal{C}_{P}$, and $\overline{(b)} \underline{e}_{P}\left(\tau_{\Lambda} S_{z_{1}}\right) \simeq r_{\Gamma} P_{z}$ if $N>4$.

Proof.

(a) Follows easily from 3.1 (a).

(b) By (a), we know that $\tau_{\Lambda} S_{z_{1}} \in \underline{\mathcal{C}_{P}}$. We assert that

$(*) \quad \underline{\operatorname{Hom}}_{\Gamma}\left({ }_{\Gamma} S_{z}, \underline{e}_{P}\left(\tau_{\Lambda} S_{z_{1}}\right)\right) \neq 0$.

Indeed, by 3.1 (a), we get the following picture

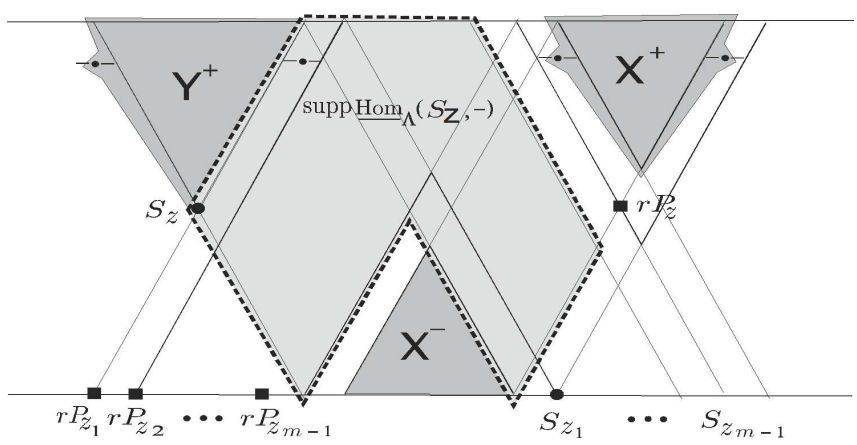

Therefore we have that $\underline{e}_{P}$ induces an isomorphism

$$
(* *) \quad \operatorname{Supp} \underline{\operatorname{Hom}}_{\Lambda}\left({ }_{\Lambda} S_{z},-\right) \cap\left(\mathrm{Y}^{+} \cup \mathrm{X}^{-}\right) \stackrel{\sim}{\rightarrow} \operatorname{Supp} \underline{\operatorname{Hom}}_{\Gamma}\left({ }_{\Gamma} S_{z},-\right) \text {, }
$$

since by $\left[9\right.$, Theorem 2.8] it follows that $\underline{e}_{P}\left({ }_{\Lambda} S_{z}\right) \simeq{ }_{\Gamma} S_{z}$. Using that $\underline{\operatorname{Hom}}_{\Lambda}\left({ }_{\Lambda} S_{z}, \tau_{\Lambda} S_{z_{1}}\right) \neq 0$ (see the picture above) and (**), we conclude that $\underline{\operatorname{Hom}}_{\Gamma}\left({ }_{\Gamma} S_{z}, \underline{e}_{P}\left(\tau_{\Lambda} S_{z_{1}}\right)\right) \neq 0$; proving $(*)$.

Consider a lifting ${ }_{S} \Gamma_{\Gamma}[0]$ of ${ }_{S} \Gamma_{\Gamma}$ to $\mathbb{Z} \mathbf{D}_{N}$ at ${ }_{\Gamma} S_{z}$. Then, by $(*)$, we get that

$$
(* * *) \quad \underline{e}_{P}\left(\tau_{\Lambda} S_{z_{1}}\right)[0] \in \operatorname{Supp}\left({ }_{\Gamma} S_{z}[0],-\right) .
$$


So, since $\tau_{\Lambda} S_{z_{1}}$ is in the bottom border of ${ }_{S} \Gamma_{\Lambda}$ and $N>4$, the isomorphism of quivers $\underline{e}_{P} \mid \mathbf{X}^{-}: \mathbf{X}^{-} \rightarrow \underline{e}_{P}\left(\mathbf{X}^{-}\right)$gives us that $\underline{e}_{P}\left(\tau_{\Lambda} S_{z_{1}}\right)[0]$ belongs to the bottom border of $\mathbb{Z} \mathbf{D}_{N}$. Furthermore, since $z$ is an insertion vertex of $Q_{\Gamma}$, we obtain by 3.5 that ${ }_{\Gamma} S_{z}[0]$ belongs to the bottom border of $\mathbb{Z} \mathbf{D}_{N}$. Then, from $(* * *)$, we have that $\nu_{D_{N}}\left(\Gamma_{\Gamma} S_{z}[0]\right)=$ $\underline{e}_{P}\left(\tau_{\Lambda} S_{z_{1}}\right)[0]$. Hence $r_{\Gamma} P_{z}[0]=\underline{e}_{P}\left(\tau_{\Lambda} S_{z_{1}}\right)[0]$ since, by [9, Proposition 3.3.], we know that $\nu_{D_{N}}\left(\Gamma_{\Gamma} S_{z}[0]\right)=r_{\Gamma} P_{z}[0]$; proving that $\underline{e}_{P}\left(\tau_{\Lambda} S_{z_{1}}\right) \simeq$ $r_{\Gamma} P_{z}$.

\section{The embedding of $k\left(\mathbb{Z} \mathbf{D}_{N}\right)$ in $k\left(\mathbb{Z} \mathbf{D}_{N+m-1}\right)$.}

Throughout this section $\Gamma$ is a trivial extension of Cartan class $\mathbf{D}_{N}, z$ is an insertion vertex of $Q_{\Gamma}$, and $\Lambda$ is the trivial extension of Cartan class $\mathbf{D}_{N+m-1}$ obtained from $\Gamma$ by inserting the cycle $C_{z}=$ $z \leftarrow z_{1} \leftarrow z_{2} \leftarrow \cdots \leftarrow z_{m-1} \leftarrow z$ at $z$. In the previous section, we described an embedding of ${ }_{S} \Gamma_{\Gamma}$ in ${ }_{S} \Gamma_{\Lambda}$. In this section, we will study the corresponding embedding between their coverings $k\left(\mathbb{Z} \mathbf{D}_{N}\right)$ and $k\left(\mathbb{Z} \mathbf{D}_{N+m-1}\right)$.

Recall that $\Gamma \simeq \operatorname{End}_{\Lambda}(P)^{o p}$ where $P$ is the projective $\Lambda$-module $\coprod_{i \in\left(Q_{\Gamma}\right)_{0}{ }_{\Lambda} P_{i}}$. In Section 3, we studied the embedding $\imath: \underline{\bmod } \Gamma \rightarrow$ $\underline{\bmod } \Lambda$ and saw that ind $\underline{\mathcal{C}_{P}}$ splits inside ${ }_{S} \Gamma_{\Lambda}$ in four convex components $\mathrm{X}^{ \pm}, \mathrm{Y}^{ \pm}$(see 3.1).

The partition $\left\{\mathrm{X}^{-}, \mathrm{X}^{+}, \mathrm{Y}^{-}, \mathrm{Y}^{+}\right\}$of ind $\underline{\mathcal{C}_{P}}$ induces, through the equivalence $\underline{e}_{P}: \underline{\mathcal{C}_{P}} \rightarrow \underline{\bmod } \Gamma$, the partition in ${ }_{S} \Gamma_{\Gamma}$ indicated in the following picture where $x:=\underline{e}_{P}\left(\tau_{\Lambda} S_{z_{1}}\right) \simeq r_{\Gamma} P_{z}$ (see 3.6).

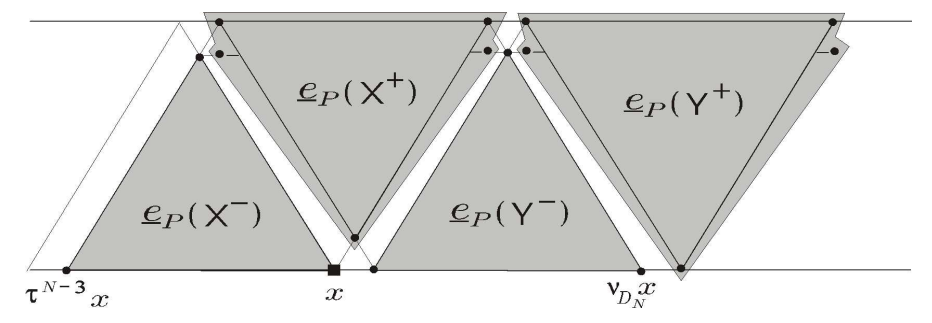

So, the partition $\left\{\underline{e}_{P}\left(\mathrm{X}^{-}\right), \underline{e}_{P}\left(\mathrm{X}^{+}\right), \underline{e}_{P}\left(\mathrm{Y}^{-}\right), \underline{e}_{P}\left(\mathrm{Y}^{+}\right)\right\}$of ${ }_{S} \Gamma_{\Gamma}$ depends on the insertion vertex $z$ of $Q_{\Gamma}$, this is, of $r_{\Gamma} P_{z}$.

On the other hand, the embedding $\imath: \underline{\bmod } \Gamma \rightarrow \underline{\bmod } \Lambda$ induces a map 
denoted by the same symbol $\imath:{ }_{S} \Gamma_{\Gamma} \rightarrow{ }_{S} \Gamma_{\Lambda}$. Indeed, let $\alpha: M \rightarrow N$ be an arrow of ${ }_{S} \Gamma_{\Gamma}$. Then, by 3.1 and 3.4 , there exists a unique sectional path in ${ }_{S} \Gamma_{\Lambda}$ starting at $\imath(M)$ and ending at $\imath(N)$. Hence we can define $\imath(\alpha)$ to be this sectional path. It is not difficult to see that, if $\rho$ is a mesh relation in ${ }_{S} \Gamma_{\Gamma}$ then $\imath(\rho)$ is zero in $k\left({ }_{S} \Gamma_{\Lambda}\right)$. Therefore the map $\imath$ induces a full and faithful functor, denoted also by $\imath: k\left({ }_{S} \Gamma_{\Gamma}\right) \rightarrow k\left({ }_{S} \Gamma_{\Lambda}\right)$. Let $\pi: \mathbb{Z} \mathbf{D}_{N} \rightarrow{ }_{S} \Gamma_{\Gamma}$ and $\pi^{\prime}: \mathbb{Z} \mathbf{D}_{N+m-1} \rightarrow$ ${ }_{S} \Gamma_{\Lambda}$ be the universal coverings of ${ }_{S} \Gamma_{\Gamma}$ and ${ }_{S} \Gamma_{\Lambda}$ respectively. In this section, we will define a functor $\Phi: k\left(\mathbb{Z} \mathbf{D}_{N}\right) \rightarrow k\left(\mathbb{Z} \mathbf{D}_{N+m-1}\right)$ making the following diagram commutative

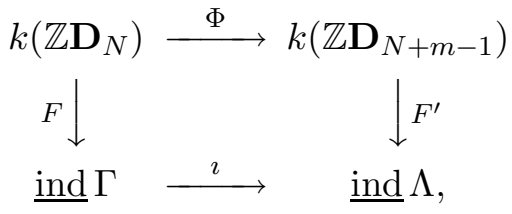

where $F$ and $F^{\prime}$ are the well behaved functors induced by the coverings $\pi$ and $\pi^{\prime}$ respectively. In order to describe such a functor $\Phi$, we introduce some notation.

\subsection{The $\mathcal{X}, \mathcal{Y}$ partition in $\mathbb{Z} \mathbf{D}_{N}$.}

Let $X$ be a subset of vertices in $\mathbb{Z} \mathbf{D}_{N}$. We denote by Conv $(X)$ the convex closure of $X$ in $\mathbb{Z} \mathbf{D}_{N}$, which is the smallest convex subquiver of $\mathbb{Z} \mathbf{D}_{N}$ containing the set $X$. Furthermore, given a vertex $x \in \mathbb{Z} \mathbf{D}$, the section of $\mathbb{Z} \mathbf{D}_{N}$ starting at $x$ will be denoted by $\overrightarrow{\mathcal{S}}_{x}$.

Definition 4.1. For a given vertex $x$ of the bottom border of $\mathbb{Z} \mathbf{D}_{N}$, we define the following vertices and the full convex subquivers of $\mathbb{Z} \mathbf{D}_{N}$.

(a) $\beta_{S}(x)$ (resp. $\beta_{M}(x)$ ) is the vertex of the section $\overrightarrow{\mathcal{S}}_{x}$ which is in the top (resp. middle) border of $\mathbb{Z} \mathbf{D}_{N}$.

(b) $\mathcal{X}_{x}^{-}:=\operatorname{Conv}\left(\left\{\tau^{-1} \nu_{\mathbf{D}_{N}}^{-1}(x), x\right\}\right)$.

(c) $\mathcal{X}_{x}^{+}:=\operatorname{Conv}\left(\left\{\tau^{-1} \nu_{\mathbf{D}_{N}}^{-1}(x), \beta_{S}(x), \beta_{M}(x)\right\}\right) \backslash \mathcal{X}_{x}^{-}$.

(d) $\mathcal{Y}_{x}^{-}:=\operatorname{Conv}\left(\left\{\tau^{-1} x, \nu_{\mathbf{D}_{N}}(x)\right\}\right)$.

(e) $\mathcal{Y}_{x}^{+}:=\operatorname{Conv}\left(\left\{\tau^{-1} x, \beta_{S}\left(\tau^{-1} \nu_{\mathbf{D}_{N}}(x)\right), \beta_{M}\left(\tau^{-1} \nu_{\mathbf{D}_{N}}(x)\right)\right\}\right) \backslash \mathcal{Y}_{x}^{-}$. 
In the following picture, the shape of these subquivers of $\mathbb{Z} \mathbf{D}_{N}$ can be seen. We also remark that $\tau^{-1} \nu_{\mathbf{D}_{N}}^{-1}(x)=\tau^{N-3}(x)$.

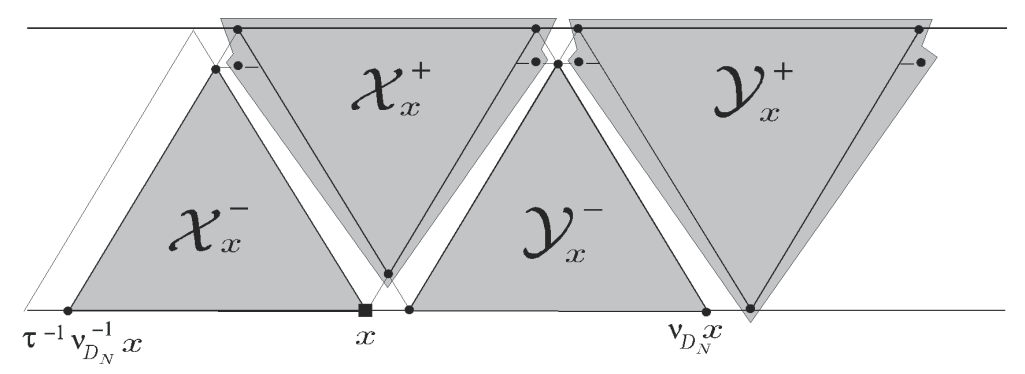

Following [4], we recall that $m_{\mathbb{Z} \mathbf{D}_{N}}=2 N-3$, where $m_{\mathbb{Z} \mathbf{D}_{N}}$ is the Loewy Length in the mesh category $k\left(\mathbb{Z} \mathbf{D}_{N}\right)$. For any vertex $u$ of $\mathbb{Z} \mathbf{D}_{N}$ and any integer $i$, we set $u[i]:=\tau^{-i(2 N-3)}(u)$. Moreover, if $\mathcal{Z}$ is a subquiver of $\mathbb{Z} \mathbf{D}_{N}$ and $i$ is any integer, we will consider the $i$-shifted subquiver $\mathcal{Z}[i]:=\tau^{-i(2 N-3)}(\mathcal{Z})$ of $\mathbb{Z} \mathbf{D}_{N}$.

Definition 4.2. For a given vertex $x$ of the bottom border of $\mathbb{Z} \mathbf{D}_{N}$ and any integer $i$, we set: $C_{x}^{+}[i]:=\mathcal{X}_{x}^{+}[i] \cup \mathcal{Y}_{x}^{+}[i], C_{x}^{-}[i]:=\mathcal{X}_{x}^{-}[i] \cup \mathcal{Y}_{x}^{-}[i]$ and $C_{x}^{ \pm}:=\cup_{i \in \mathbb{Z}} C_{x}^{ \pm}[i]$. Thus, $\left\{C_{x}^{-}[i], C_{x}^{+}[i]: i \in \mathbb{Z}\right\}$ is a partition of $\mathbb{Z} \mathbf{D}_{N}$.

Remark 4.3. (1) Since $z$ is an insertion vertex of $Q_{\Gamma}$, we get by 3.5 that if $N>4$ then the fiber $\pi^{-1}\left(r_{\Gamma} P_{z}\right)$ is contained in the bottom border of $\mathbb{Z} \mathbf{D}_{N}$, where $\pi: \mathbb{Z} \mathbf{D}_{N} \rightarrow{ }_{S} \Gamma_{\Gamma}$ is the universal covering of ${ }_{S} \Gamma_{\Gamma}$. In case $N=4$, we choose $\pi$ in such a way that $\pi^{-1}\left(r_{\Gamma} P_{z}\right)$ belongs to the bottom border of $\mathbb{Z} \mathbf{D}_{4}$.

(2) The Nakayama permutation $\nu_{\mathbf{D}_{N}}:\left(\mathbb{Z} \mathbf{D}_{N}\right)_{0} \rightarrow\left(\mathbb{Z} \mathbf{D}_{N}\right)_{0}$ commutes with the translation $\tau$ of $\mathbb{Z} \mathbf{D}_{N}$. Thus, $\nu_{\mathbf{D}_{N}}$ induces a permutation on $\left({ }_{S} \Gamma_{\Gamma}\right)_{0}$ which is also denoted by $\nu_{\mathbf{D}_{N}}$.

Lemma 4.4. Let $z$ be an insertion vertex of $Q_{\Gamma}, \pi: \mathbb{Z} \mathbf{D}_{N} \rightarrow{ }_{S} \Gamma_{\Gamma}$ be the universal covering of ${ }_{S} \Gamma_{\Gamma}$ and $\left\{\mathrm{X}^{-}, \mathrm{X}^{+}, \mathrm{Y}^{-}, \mathrm{Y}^{+}\right\}$be the partition of ind $\underline{\mathcal{C}_{P}}$ in ${ }_{S} \Gamma_{\Lambda}$ (see 3.1).

(a) If $x \in \pi^{-1}\left(r_{\Gamma} P_{z}\right)$, then $\left.\imath \pi\right|_{\mathcal{X}_{x}^{ \pm}}: \mathcal{X}_{x}^{ \pm} \rightarrow \mathrm{X}^{ \pm}$and $\left.\imath \pi\right|_{\mathcal{Y}_{x}^{ \pm}}: \mathcal{Y}_{x}^{ \pm} \rightarrow$ $\mathrm{Y}^{ \pm}$are quiver isomorphisms. 
(b) Let ${ }_{S} \Gamma_{\Gamma}[0]$ be a lifting of ${ }_{S} \Gamma_{\Gamma}$ to $\mathbb{Z}_{\mathbf{D}_{N}}$ at $\tau^{-1} \nu_{\mathbf{D}_{N}}^{-1}\left(r_{\Gamma} P_{z}\right)$. If $x=r_{\Gamma} P_{z}[0]$ then ${ }_{S} \Gamma_{\Gamma}[0]=C_{x}^{+}[0] \cup C_{x}^{-}[0]$.

Proof. It follows easily from definitions and 3.1.

\subsection{Definition of the functor $\Phi: k\left(\mathbb{Z} \mathbf{D}_{N}\right) \rightarrow k\left(\mathbb{Z} \mathbf{D}_{N+m-1}\right)$.}

In what follows, we define a functor $\Phi: k\left(\mathbb{Z} \mathbf{D}_{N}\right) \rightarrow k\left(\mathbb{Z} \mathbf{D}_{N+m-1}\right)$ which is a "lifting" of the embedding $\imath: \underline{\text { ind }} \Gamma \rightarrow \underline{\text { ind }} \Lambda$. In order to get some feeling about the correct definition of $\Phi$, we start by looking at the following situation. Consider the partition $\left\{\underline{e}_{P}\left(\mathrm{X}^{-}\right), \underline{e}_{P}\left(\mathrm{X}^{+}\right)\right.$, $\left.\underline{e}_{P}\left(\mathrm{Y}^{-}\right), \underline{e}_{P}\left(\mathrm{Y}^{+}\right)\right\}$of ${ }_{S} \Gamma_{\Gamma}$. Let $U$ and $V$ in $\underline{\operatorname{ind}} \Gamma$ as in the following picture.

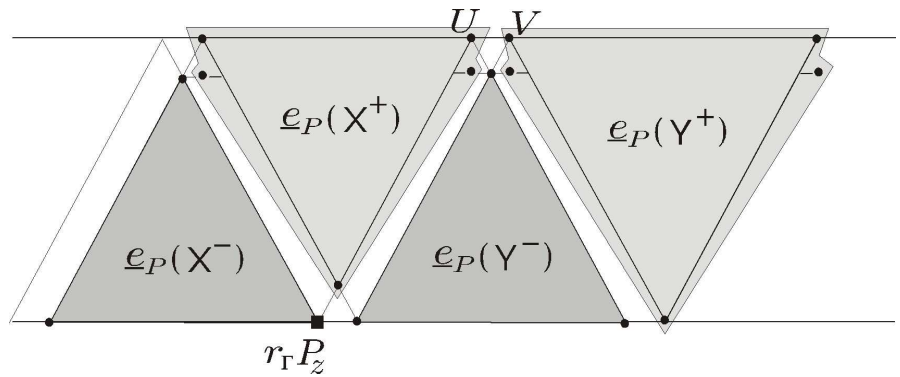

Then $\imath(U)$ (resp. $\imath(V)$ ) is either in the top or in the middle border of ${ }_{S} \Gamma_{\Lambda}$. By fixing $\imath(U)$ in the top border of ${ }_{S} \Gamma_{\Lambda}$, we assert that $\imath(U)$ and $\imath(V)$ belong to the same border if and only if $m-1$ is even. Indeed, $\underline{\operatorname{Hom}}_{\Gamma}(U, V)=0$ since $U=\tau V$; and so $\underline{\operatorname{Hom}}_{\Lambda}(\imath(U), \imath(V))=0$. Thus, by using the shape of $\operatorname{Supp} \underline{\operatorname{Hom}}_{\Lambda}(\imath(U),-)$ in ${ }_{S} \Gamma_{\Lambda}$ (see 1.11 and [8, Proposition 3.3]), we get the assertion. Then, motivated by this property of the functor $\imath$, we introduce the following definitions. To do that, we will make use of the automorphism $\sigma \in \operatorname{Aut}\left(\mathbb{Z} \mathbf{D}_{N}\right)$ which is defined as follows: $\sigma(p, N-1):=(p, N), \sigma(p, N):=(p, N-1)$ and $\sigma(p, q):=(p, q)$ in case $1 \leq q \leq N-2$. That is, $\sigma$ sends the top border of $\mathbb{Z} \mathbf{D}_{N}$ to the middle one and fix the other vertices of $\mathbb{Z} \mathbf{D}_{N}$.

Let $x$ be a vertex of the bottom border of $\mathbb{Z} \mathbf{D}_{N}$ and $y$ be a vertex of the top border of $\mathbb{Z} \mathbf{D}_{N}$. By using the quivers $C_{x}^{-}$and $C_{x}^{+}$(see 4.2 ), we will define a $k$-linear fully faithful functor $\Phi=\Phi_{m-1, x, y}$ : 
$k\left(\mathbb{Z} \mathbf{D}_{N}\right) \rightarrow k\left(\mathbb{Z} \mathbf{D}_{N+m-1}\right)$. Such a functor depends on the automorphism $\sigma \in \operatorname{Aut}\left(\mathbb{Z} \mathbf{D}_{N}\right)$ and on two functions $\Phi_{m-1, x, \mathcal{X}^{+}}, \Phi_{m-1, x, \mathcal{Y}^{+}}$: $\left(\mathbb{Z} \mathbf{D}_{N}\right)_{0} \rightarrow\left(\mathbb{Z} \mathbf{D}_{N+m-1}\right)_{0}$. So, we start by defining these auxiliary functions as follows.

Definition 4.5. The functions $\Phi_{m-1, x, \mathcal{X}^{+}}$and $\Phi_{m-1, x, \mathcal{Y}^{+}}$.

(a) $\operatorname{For}(p, q) \in C_{x}^{-}[0] \cup C_{x}^{+}[0]$, we set

$$
\begin{gathered}
\Phi_{m-1, x, \mathcal{X}^{+}}(p, q):= \begin{cases}(p, q) & \text { if }(p, q) \in \mathcal{X}_{x}^{-}, \\
(p, q+m-1) & \text { if }(p, q) \in \mathcal{X}_{x}^{+}, \\
(p+m-1, q) & \text { if }(p, q) \in \mathcal{Y}_{x}^{-}, \\
\sigma^{m-1}(p+m-1, q+m-1) & \text { if }(p, q) \in \mathcal{Y}_{x}^{+}\end{cases} \\
\Phi_{m-1, x, \mathcal{Y}^{+}}(p, q):= \begin{cases}(p, q) & \text { if }(p, q) \in \mathcal{X}_{x}^{-}, \\
\sigma^{m-1}(p, q+m-1) & \text { if }(p, q) \in \mathcal{X}_{x}^{+}, \\
(p+m-1, q) & \text { if }(p, q) \in \mathcal{Y}_{x}^{-}, \\
(p+m-1, q+m-1) & \text { if }(p, q) \in \mathcal{Y}_{x}^{+} .\end{cases}
\end{gathered}
$$

(b) For $t \in C_{x}^{ \pm}[i]$ with $i \neq 0$, we set

$$
\begin{aligned}
& \Phi_{m-1, x, \mathcal{X}^{+}}(t):=\Phi_{m-1, x, \mathcal{X}^{+}}(t[-i])[i], \\
& \Phi_{m-1, x, \mathcal{Y}^{+}}(t):=\Phi_{m-1, x, \mathcal{Y}^{+}}(t[-i])[i] .
\end{aligned}
$$

Definition 4.6. The function $\Phi=\Phi_{m-1, x, y}$ on the vertices of $\mathbb{Z} \mathbf{D}_{N}$.

$$
\Phi(t):= \begin{cases}\Phi_{m-1, x, \mathcal{X}^{+}}(t) & \text { if } y \in \cup_{i \in \mathbf{Z}} \mathcal{X}_{x}^{+}[i] \\ \Phi_{m-1, x, \mathcal{Y}^{+}}(t) & \text { if } y \in \cup_{i \in \mathbf{Z}} \mathcal{Y}_{x}^{+}[i]\end{cases}
$$

In the following picture, we illustrate the shape of the set $\Phi\left(\left(\mathbb{Z} \mathbf{D}_{N}\right)_{0}\right)$

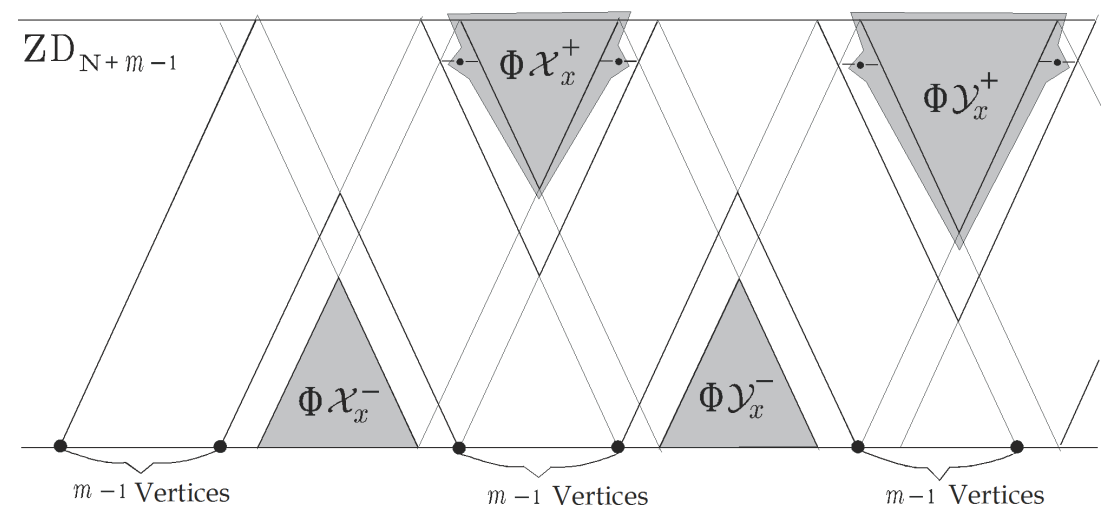


Definition 4.7. The function $\Phi=\Phi_{m-1, x, y}$ on the arrows of $\mathbb{Z} \mathbf{D}_{N}$. Let $w \stackrel{\alpha}{\rightarrow} z$ be an arrow of $\mathbb{Z} \mathbf{D}_{N}$. By 4.6, it is not difficult to see that there exists a unique sectional path $\gamma$ in $\mathbb{Z} \mathbf{D}_{N+m-1}$ starting at $\Phi(w)$ and ending at $\Phi(z)$; and so we set $\Phi(\alpha):=\gamma$.

Remark 4.8. if $\alpha$ belongs to one of the components $\mathcal{X}^{ \pm}[i], \mathcal{Y}^{ \pm}[i]$ for some integer $i$, then $\Phi(\alpha)$ is an arrow in $\mathbb{Z} \mathbf{D}_{N+m-1}$. Moreover, it is not difficult to see that if $\rho$ is a mesh relation in $\mathbb{Z} \mathbf{D}_{N}$ then $\Phi(\rho)$ is zero in $k\left(\mathbb{Z} \mathbf{D}_{N+m-1}\right)$. Thus, we get a k-linear fully faithful functor, denoted also by $\Phi$

$$
\Phi=\Phi_{m-1, x, y}: k\left(\mathbb{Z} \mathbf{D}_{N}\right) \rightarrow k\left(\mathbb{Z} \mathbf{D}_{N+m-1}\right) .
$$

Lemma 4.9. Let $z$ be an insertion vertex of $Q_{\Gamma}, \pi: \mathbb{Z} \mathbf{D}_{N} \rightarrow{ }_{S} \Gamma_{\Gamma}$ be the universal covering of ${ }_{S} \Gamma_{\Gamma}$ (as fixed in 4.3), $\pi^{\prime}: \mathbb{Z}_{N+m-1} \rightarrow{ }_{S} \Gamma_{\Lambda}$ be the universal covering of ${ }_{S} \Gamma_{\Lambda}$ and $y$ be a vertex of the top border of $\mathbb{Z} \mathbf{D}_{N}$. Let ${ }_{S} \Gamma_{\Gamma}[0]$ be a lifting of ${ }_{S} \Gamma_{\Gamma}$ to $\mathbb{Z}_{\mathbf{D}_{N}}$ at $\tau^{-1} \nu_{\mathbf{D}_{N}}^{-1}\left(r_{\Gamma} P_{z}\right)$ and take $x:=r_{\Gamma} P_{z}[0]$ as the fixed vertex in the bottom border of $\mathbb{Z} \mathbf{D}_{N}$. We fix a lifting ${ }_{S} \Gamma_{\Lambda}[0]$ of ${ }_{S} \Gamma_{\Lambda}$ to $\mathbf{Z D}_{N+m-1}$ at $\tau^{-1} r_{\Lambda} P_{z_{1}}$ in such a way that $\tau^{-1} r_{\Lambda} P_{z_{1}}[0]=\tau^{-1} \nu_{\mathbf{D}_{N+m-1}}^{-1}\left(\Phi\left(r_{\Gamma} P_{z}[0]\right)\right)$. Then, the functor

$$
\Phi=\Phi_{m-1, r_{\Gamma} P_{z}[0], y}: k\left(\mathbb{Z} \mathbf{D}_{N}\right) \rightarrow k\left(\mathbb{Z} \mathbf{D}_{N+m-1}\right)
$$

has the following properties:

(a) the maps $\left.\pi^{\prime} \Phi\right|_{\mathcal{X}_{r_{\Gamma} P_{z}[0]}^{ \pm}}: \mathcal{X}_{r_{\Gamma} P_{z}[0]}^{ \pm} \rightarrow \mathrm{X}^{ \pm}$and $\left.\pi^{\prime} \Phi\right|_{\mathcal{Y}_{r_{\Gamma} P_{z}[0]}^{ \pm}}: \mathcal{Y}_{r_{\Gamma} P_{z}[0]}^{ \pm} \rightarrow \mathrm{Y}^{ \pm}$are quiver isomorphisms,

(b) ${ }_{S} \Gamma_{\Lambda}[0]=C_{\Phi\left(r_{\Gamma} P_{z}[0]\right)}^{+}[0] \cup C_{\Phi\left(r_{\Gamma} P_{z}[0]\right)}^{-}[0]$,

(c) $\Phi\left(C_{r_{\Gamma} P_{z}[0]}^{ \pm}[0]\right) \subseteq C_{\Phi\left(r_{\Gamma} P_{z}[0]\right)}^{ \pm}[0]$.

Proof. It follows directly from the definitions and 4.4.

Remark 4.10. In the following two pictures, we illustrate 4.9.

(a) The partition of ${ }_{S} \Gamma_{\Gamma}[0]$ induced by the vertex $x:=r_{\Gamma} P_{z}[0]$. 


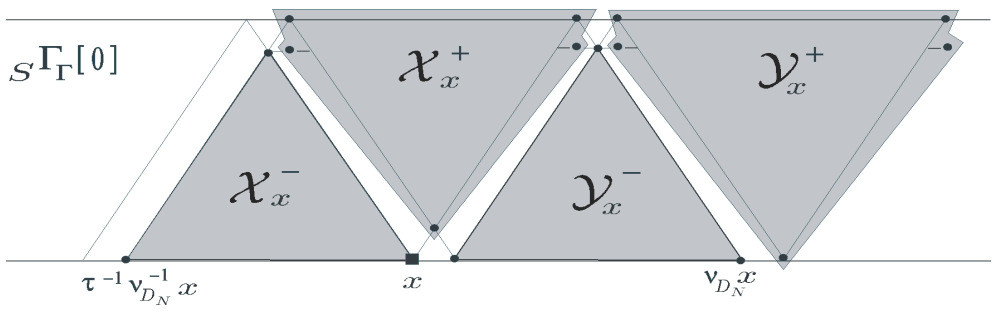

(b) The partition of ${ }_{S} \Gamma_{\Lambda}[0]$ which is compatible with the functor $\Phi$.

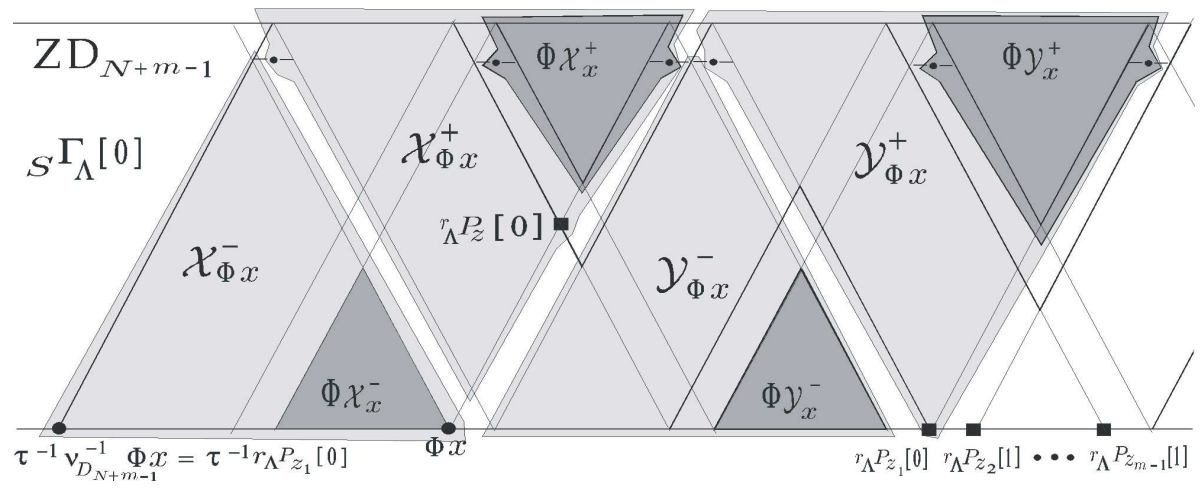

Theorem 4.11. Let $\Gamma$ be a trivial extension of Cartan class $\mathbf{D}_{N}, z$ be an insertion vertex of $Q_{\Gamma}$ and $\Lambda$ be the trivial extension obtained from $\Gamma$ by inserting the minimal cycle $C_{z}=z \leftarrow z_{1} \leftarrow \cdots \leftarrow z_{m-1} \leftarrow z$ at z. Fix $i_{0} \in \mathbb{N}$ such that $i_{0}:=1$ if $\Gamma$ is of Class $I$, and $i_{0}:=3$ if $\Gamma$ is either of Class II or III (see 1.9). Choose the universal coverings $\pi: \mathbb{Z D}_{N} \rightarrow{ }_{S} \Gamma_{\Gamma}$ and $\pi^{\prime}: \mathbb{Z}_{N+m-1} \rightarrow{ }_{S} \Gamma_{\Lambda}$ as in 4.9 and such that $h_{\mathbb{Z} \mathbf{D}_{N}}\left(\pi^{-1}\left(r_{\Gamma} P_{i_{0}}\right)\right)=N-1$. Consider also the liftings and the full and faithful functor

$$
\Phi_{z, i_{0}}:=\Phi_{m-1, r_{\Gamma} P_{z}[0], y}: k\left(\mathbb{Z} \mathbf{D}_{N}\right) \rightarrow k\left(\mathbb{Z} \mathbf{D}_{N+m-1}\right),
$$

fixed in 4.9, where $y:=r_{\Gamma} P_{i_{0}}[0]$.

If $F^{\prime}: k\left(\mathbb{Z} \mathbf{D}_{N+m-1}\right) \rightarrow \underline{\text { ind }} \Lambda$ is a well behaved functor induced by the universal covering $\pi^{\prime}: \overline{\mathbb{Z}}_{N+m-1} \rightarrow{ }_{S} \Gamma_{\Lambda}$, then 
(a) there is a well behaved functor $F: k\left(\mathbb{Z} \mathbf{D}_{N}\right) \rightarrow$ ind $\Gamma$, induced by the universal covering $\pi: \mathbb{Z} \mathbf{D}_{N} \rightarrow{ }_{S} \Gamma_{\Gamma}$, making the following diagram

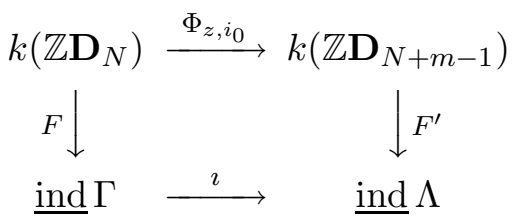

commutative, where $\imath$ is the fully faithful functor defined in Section 3;

(b) for any vertex $t \in\left(Q_{\Gamma}\right)_{0}$, the functor $\Phi_{z, i_{0}}$ satisfies:

(b1) $\Phi_{z, i_{0}}\left(r_{\Gamma} P_{t}[0]\right)= \begin{cases}r_{\Lambda} P_{t}[0] & \text { if } t \neq z \\ \tau\left({ }_{\Lambda} S_{z_{1}}[0]\right) & \text { if } t=z\end{cases}$

(b2) $\Phi_{z, i_{0}}\left({ }_{\Gamma} S_{t}[0]\right)={ }_{\Lambda} S_{t}[0]$.

Proof. For the sake of simplicity, we set $\Phi:=\Phi_{z, i_{0}}$.

(a) Firstly, we prove that $\pi^{\prime} \Phi=\imath \pi$. To do that, we consider the following functions:

$$
\begin{aligned}
F_{1}=\left.\imath \pi\right|_{\mathcal{X}_{r_{\Gamma} P_{z}[0]}^{-}} & F_{2}=\left.\imath \pi\right|_{\mathcal{Y}_{r_{\Gamma} P_{z}[0]}^{-}}, \\
G_{1}=\left.\pi^{\prime} \Phi\right|_{\mathcal{X}_{r_{\Gamma} P_{z}[0]}^{-}} & G_{2}=\left.\pi^{\prime} \Phi\right|_{\mathcal{Y}_{r_{\Gamma} P_{z}[0]}^{-}} \\
F_{3}=\left.\imath \pi\right|_{\mathcal{X}_{r_{\Gamma} P_{z}[0]}^{+}} & F_{4}=\left.\imath \pi\right|_{\mathcal{Y}_{r_{\Gamma} P_{z}[0]}^{+}}, \\
G_{3}=\left.\pi^{\prime} \Phi\right|_{\mathcal{X}_{r_{\Gamma} P_{z}[0]}^{+}} & G_{4}=\left.\pi^{\prime} \Phi\right|_{\mathcal{Y}_{r_{\Gamma} P_{z}[0]}^{+}}
\end{aligned}
$$

By 4.4 and 4.9 , we get that $F_{i}$ and $G_{i}$ are quiver isomorphisms for any $i$. Hence

$$
\begin{array}{ll}
G_{1}^{-1} F_{1} \in \operatorname{Aut}\left(\mathcal{X}_{r_{\Gamma} P_{z}[0]}^{-}\right)=\{1\}, & G_{2}^{-1} F_{2} \in \operatorname{Aut}\left(\mathcal{Y}_{r_{\Gamma} P_{z}[0]}^{-}\right)=\{1\}, \\
G_{3}^{-1} F_{3} \in \operatorname{Aut}\left(\mathcal{X}_{r_{\Gamma} P_{z}[0]}^{+}\right)=\{1, \sigma\}, & G_{4}^{-1} F_{4} \in \operatorname{Aut}\left(\mathcal{Y}_{r_{\Gamma} P_{z}[0]}^{+}\right)=\{1, \sigma\} .
\end{array}
$$

Then $F_{1}=G_{1}, F_{2}=G_{2}$; and to obtain $\pi^{\prime} \Phi=\imath \pi$, we have to prove that $F_{i}=G_{i}$ for $i=3,4$. By definition we have

$$
\Phi= \begin{cases}\Phi_{m-1, r_{\Gamma} P_{z}[0], \mathcal{X}+} & \text { if } r_{\Gamma} P_{i_{0}}[0] \in \mathcal{X}_{r_{\Gamma} P_{z}[0]}^{+}, \\ \Phi_{m-1, r_{\Gamma} P_{z}[0], \mathcal{Y}^{+}} & \text {if } r_{\Gamma} P_{i_{0}}[0] \in \mathcal{Y}_{r_{\Gamma} P_{z}[0]}^{+}\end{cases}
$$


Assume that $r_{\Gamma} P_{i_{0}}[0] \in \mathcal{X}_{r_{\Gamma} P_{z}[0]}^{+}$(for the other case, the proof is very similar). Then $\Phi=\Phi_{m-1, r_{\Gamma} P_{z}[0], \mathcal{X}+}$.

•) $F_{3}=G_{3}$.

Indeed, since $r_{\Gamma} P_{i_{0}}[0]$ is in the top border of $\mathbb{Z} \mathbf{D}_{N}$ and $r_{\Gamma} P_{i_{0}}[0] \in$ $\mathcal{X}_{r_{\Gamma} P_{z}[0]}^{+}$, we get that $\Phi\left(r_{\Gamma} P_{i_{0}}[0]\right) \in \mathrm{X}^{+}[0]$ with $\Phi\left(r_{\Gamma} P_{i_{0}}[0]\right)$ in the top border of $\mathbb{Z} \mathbf{D}_{N+m-1}$. Furthermore, by [9, Theorem 2.8], we know that $\imath\left(r_{\Gamma} P_{i_{0}}\right) \simeq r_{\Lambda} P_{i_{0}}$; and hence $r_{\Lambda} P_{i_{0}}[0] \in \mathrm{X}^{+}[0]$ since $r_{\Gamma} P_{i_{0}}[0] \in \mathcal{X}_{r_{\Gamma} P_{z}[0]}^{+}$. So, using that $r_{\Lambda} P_{i_{0}}[0]$ belongs to $\mathrm{X}^{+}[0]$ and to the top border of $\mathbb{Z} \mathbf{D}_{N+m-1}$, we obtain that $\Phi\left(r_{\Gamma} P_{i_{0}}[0]\right)=r_{\Lambda} P_{i_{0}}[0]$ since $\imath\left(r_{\Gamma} P_{i_{0}}\right) \simeq$ $r_{\Lambda} P_{i_{0}}$ and $\underline{e}_{P} \mid \mathrm{X}^{+}: \mathrm{X}^{+} \rightarrow \pi \mathcal{X}_{r_{\Gamma} P_{z}[0]}^{+}$is an isomorphism of quivers. Therefore $\pi^{\prime} \Phi\left(r_{\Gamma} P_{i_{0}}[0]\right)=r_{\Lambda} P_{i_{0}}$; and hence $G_{3}^{-1} F_{3}\left(r_{\Gamma} P_{i_{0}}[0]\right)=$ $r_{\Gamma} P_{i_{0}}[0]$. Then $G_{3}^{-1} F_{3}=1$ since the automorphism $\sigma$ sends the top border of $\mathbb{Z} \mathbf{D}_{N}$ to the middle border of $\mathbb{Z} \mathbf{D}_{N}$.

-) $F_{4}=G_{4}$.

Indeed, let $x=r_{\Gamma} P_{z}[0]$. Then $\beta_{S}(x) \in \mathcal{X}_{x}^{+}$and $\beta_{M}\left(\tau^{-1} x\right) \in \mathcal{Y}_{x}^{+}$.

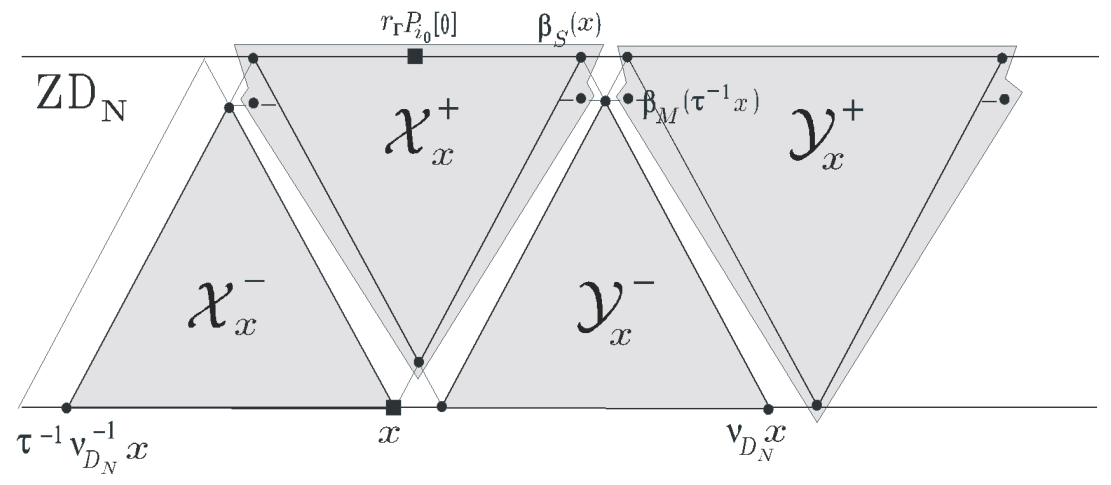

Let $F_{\pi}: k\left(\mathbb{Z} \mathbf{D}_{N}\right) \rightarrow \underline{\text { ind }} \Gamma$ be a well-behaved functor induced by the universal covering $\pi: \mathbb{Z}_{N} \rightarrow{ }_{S} \Gamma_{\Gamma}$. From [8, 3.2 and 3.3], we have that

$$
F_{\pi}: k\left(\mathbb{Z D}_{N}\right)\left(\beta_{S}(x), \beta_{M}\left(\tau^{-1} x\right)\right) \rightarrow \underline{\operatorname{Hom}}_{\Gamma}\left(\pi \beta_{S}(x), \pi \beta_{M}\left(\tau^{-1} x\right)\right)
$$


is a $k$-linear isomorphism. On the other hand, we know that

$$
\underline{\operatorname{Hom}}_{\Gamma}\left(\pi \beta_{S}(x), \pi \beta_{M}\left(\tau^{-1} x\right)\right) \simeq \underline{\operatorname{Hom}}_{\Lambda}\left(\imath \pi \beta_{S}(x), \imath \pi \beta_{M}\left(\tau^{-1} x\right)\right) ;
$$

and by these isomorphisms, we get $\underline{\operatorname{Hom}}_{\Lambda}\left(\imath \pi \beta_{S}(x), \imath \pi \beta_{M}\left(\tau^{-1} x\right)\right) \neq 0$ since, by the preceding picture, we know that $k\left(\mathbb{Z} \mathbf{D}_{N}\right)\left(\beta_{S}(x), \beta_{M}\left(\tau^{-1} x\right)\right)$ $\neq 0$. Using that $F_{3}=G_{3}$, we have that $\imath \pi\left(\beta_{S}(x)\right)=\pi^{\prime} \Phi\left(\beta_{S}(x)\right)$. Thus

$$
(* *) \quad \imath \pi\left(\beta_{M}\left(\tau^{-1} x\right)\right) \in \operatorname{Supp} \underline{\operatorname{Hom}}_{\Lambda}\left(\pi^{\prime} \Phi\left(\beta_{S}(x)\right),-\right) .
$$

Furthermore, by [8, Proposition 3.3], it follows that the universal covering $\pi^{\prime}: \mathbb{Z}_{N+m-1} \rightarrow{ }_{S} \Gamma_{\Lambda}$ induces a bijection

$$
\operatorname{Supp} k\left(\mathbb{Z} \mathbf{D}_{N+m-1}\right)\left(\Phi\left(\beta_{S}(x)\right),-\right) \stackrel{\sim}{\rightarrow} \operatorname{Supp} \underline{\operatorname{Hom}}_{\Lambda}\left(\pi^{\prime} \Phi\left(\beta_{S}(x)\right),-\right) \text {. }
$$

Moreover, by 1.11, we know the shape of $\operatorname{Supp} k\left(\mathbb{Z} \mathbf{D}_{N+m-1}\right)\left(\Phi\left(\beta_{S}(x)\right),-\right)$ in $\mathbb{Z} \mathbf{D}_{N+m-1}$. Then, by $(* *)$, we conclude that

$$
\imath \pi\left(\beta_{M}\left(\tau^{-1} x\right)\right)=\pi^{\prime} \sigma^{m-1} \beta_{M}\left(\Phi\left(\tau^{-1} x\right)\right) .
$$

By the definition of $\Phi$, we get that $\Phi\left(\beta_{M}\left(\tau^{-1} x\right)\right)=\sigma^{m-1} \beta_{M}\left(\Phi\left(\tau^{-1} x\right)\right)$. Then $\imath \pi\left(\beta_{M}\left(\tau^{-1} x\right)\right)=\pi^{\prime} \Phi\left(\beta_{M}\left(\tau^{-1} x\right)\right)$ and hence $G_{4}^{-1} F_{4}\left(\beta_{M}\left(\tau^{-1} x\right)\right)=$ $\beta_{M}\left(\tau^{-1} x\right)$. Thus $G_{4}^{-1} F_{4}=1$ since the automorphism $\sigma$ sends the middle border of $\mathbb{Z} \mathbf{D}_{N}$ to the top one; proving that $\imath \pi=\pi^{\prime} \Phi$.

Let $F^{\prime}: k\left(\mathbb{Z} \mathbf{D}_{N+m-1}\right) \rightarrow$ ind $\Lambda$ be a well behaved functor induced by the universal covering $\pi^{\prime}: \mathbb{Z} \mathbf{D}_{N+m-1} \rightarrow{ }_{S} \Gamma_{\Lambda}$. Since $\imath \pi=\pi^{\prime} \Phi$. It is clear how to define $F$ on the vertices of $\mathbb{Z} \mathbf{D}_{N}$ (just take $F:=\pi$ on $\left.\left(\mathbb{Z} \mathbf{D}_{N}\right)_{0}\right)$. Now, we define $F$ on the arrows of $\mathbb{Z} \mathbf{D}_{N}$. For an arrow $x \stackrel{\alpha}{\rightarrow} y$ of $\mathbb{Z} \mathbf{D}_{N}$, we set $F(\alpha):=\underline{e}_{P} F^{\prime} \Phi(\alpha)$, where $\underline{e}_{P}=\underline{\operatorname{Hom}}_{\Lambda}(P,-)$ : $\mathcal{C}_{P} \stackrel{\sim}{\rightarrow} \bmod \Gamma$ is the equivalence of stable categories given in Section 3 . So, we have a functor $F: k\left(\mathbb{Z} \mathbf{D}_{N}\right) \rightarrow \underline{\text { ind }} \Gamma$. To see that $F$ is wellbehaved and makes the diagram of (a) commute, it is enough to prove that $\underline{e}_{P} F^{\prime} \Phi(\alpha)$ is irreducible in $\underline{\bmod } \Gamma$ for any arrow $x \stackrel{\alpha}{\rightarrow} y$ in $\mathbb{Z} \mathbf{D}_{N}$. Consider the partition $\left\{\mathcal{X}_{r_{\Gamma} P_{z}[0]}^{+}[j], \mathcal{X}_{r_{\Gamma} P_{z}[0]}^{-}[j], \mathcal{Y}_{r_{\Gamma} P_{z}[0]}^{+}[j], \mathcal{Y}_{r_{\Gamma} P_{z}[0]}^{-}[j]:\right.$ $j \in \mathbb{Z}\}$ of $\mathbb{Z} \mathbf{D}_{N}$, we have that $\Phi(x)$ and $\Phi(y)$ are either in the same or in different components of this partition.

If $\Phi(x)$ and $\Phi(y)$ are in the same component of the above partition. Then, by the definition of $\Phi$, we get that $\Phi(\alpha)$ is an arrow of $\mathbb{Z} \mathbf{D}_{N+m-1}$ and therefore $F^{\prime} \Phi(\alpha)$ is irreducible in $\underline{\bmod } \Lambda$. Hence, from 3.1 (b), we obtain that $\underline{e}_{P} F^{\prime} \Phi(\alpha)$ is irreducible in $\underline{\bmod } \Gamma$.

Suppose that $\Phi(x)$ and $\Phi(y)$ are not in the same component of the above partition. Then, by the definition of $\Phi$, we obtain that $\pi^{\prime}(\Phi(x))$ 
and $\pi^{\prime}(\Phi(y))$ belong to different components of ind $\underline{\mathcal{C}_{P}}$ (see 3.1 (a)). Moreover $\pi^{\prime}(\Phi(\alpha))$ is the following sectional path in ${ }_{S} \Gamma_{\Lambda}$

$$
\pi^{\prime}(\Phi(x)) \rightarrow M_{1} \rightarrow \cdots \rightarrow M_{r-1} \rightarrow \pi^{\prime}(\Phi(y))
$$

such that $M_{i} \notin \mathcal{C}_{P}$ for any $i=1,2, \cdots, r-1$. Therefore, by 3.4 (a), we have that $\underline{e}_{P} \overline{F^{\prime}} \Phi(\alpha)$ is irreducible in $\underline{\bmod } \Gamma$.

(b) Let $t \in\left(Q_{\Gamma}\right)_{0}$. We only prove (b1) since the prove of (b2) is very similar.

If $t=z$ then $\Phi\left(r_{\Gamma} P_{t}[0]\right)=\tau\left({ }_{\Lambda} S_{z_{1}}[0]\right)$. Assume that $t \neq z$. Then, by [9, Theorem 2.8], we have that $r_{\Lambda} P_{t} \in \mathcal{C}_{P}$ and $\underline{e}_{P}\left(r_{\Lambda} P_{t}\right) \simeq r_{\Gamma} P_{t}$. Hence $\imath\left(r_{\Gamma} P_{t}\right) \simeq r_{\Lambda} P_{t}$ since $\imath \underline{e}_{P} \simeq 1_{\mathcal{C}_{P}}$. On the other hand, by (a), we have that $\pi^{\prime} \Phi\left(r_{\Gamma} P_{t}[0]\right)=\imath \pi\left(r_{\Gamma} P_{t}[\overline{0]})=\imath\left(r_{\Gamma} P_{t}\right)\right.$, and then, $\pi^{\prime} \Phi\left(r_{\Gamma} P_{t}[0]\right)=$ $r_{\Lambda} P_{t}$ since $\imath\left(r_{\Gamma} P_{t}\right) \simeq r_{\Lambda} P_{t}$. Thus $\Phi\left(r_{\Gamma} P_{t}[0]\right)=r_{\Lambda} P_{t}[0]$; proving the result.

Corollary 4.12. Let $\Lambda$ be a trivial extension of Cartan class $\mathbf{D}_{N}$ with $N>4$. For a given vertex $z \in Q_{\Lambda}$, the following conditions are equivalent.

(a) $z$ is an insertion vertex of $Q_{\Lambda}$.

(b) $r P_{z}[0]$ belongs to the bottom border of $\mathbb{Z} \mathbf{D}_{N}$.

(c) $S_{z}[0]$ belongs to the bottom border of $\mathbb{Z} \mathbf{D}_{N}$.

In particular, the number of vertices $z \in Q_{\Lambda}$ such that $r P_{z}$ lifts to the bottom border of $\mathbb{Z} \mathbf{D}_{N}$ is larger than 1 and coincides with the number of insertion vertices of $Q_{\Lambda}$.

Proof. $\quad(\mathrm{b}) \Leftrightarrow(\mathrm{c})$ It follows from the equality $S_{z}[0]=\nu_{\mathbf{D}_{N}}\left(\tau^{-1} r P_{z}[0]\right)$ (see in [9, Proposition 3.3].

(a) $\Leftrightarrow$ (b) If $\Lambda$ is minimal then the equivalence follows from 2.12, 2.14 and 2.15 . In case $\Lambda$ is not minimal, we proceed by induction over the number of minimal oriented cycles of $Q_{\Lambda}$; and so by using 4.11 we get the result.

\section{Some generalities about trivial extensions of class $I I$.}

In this section, we extend 2.14 to the general case, that is, to a trivial extension $\Lambda$ of Class II (see 1.2 and 1.9). To do that, we firstly consider the case when $\Lambda$ is minimal of Class II (see 2.14). Then, by 
using 4.11, we see how the radicals of the projective modules, associated with the vertices 1,2 and 3 of $Q_{\Lambda}$, can be lifted to the universal covering. Furthermore, we also determine some special regions, in the bottom border of $\mathbb{Z} \mathbf{D}_{N}$, where the radicals of the projective modules associated with the insertion vertices of $Q_{\Lambda}$ can be lifted. So, throughout this section, $\Lambda$ will be a trivial extension of Class II.

We recall that the ordinary quiver $Q_{\Lambda}$ of $\Lambda$ is described (see 1.2 and 1.9) by drawing the quiver $Q_{\Lambda_{\min }}$ of $\Lambda_{\min }$ and small squares on some vertices of $Q_{\Lambda_{\text {min }}}$. The small square on a vertex $x_{j} \in Q_{\Lambda_{\text {min }}}$, represents a quiver $Q_{x_{j}}$ which is either $\left\{x_{j}\right\}$ or the ordinary quiver of a trivial extension $\Gamma_{x_{j}}$ of Cartan class $\mathbf{A}_{n_{j}}$ with $n_{j} \geq 2$.

Definition 5.1. For a trivial extension $\Lambda$ of Class II, it will be considered the following subquivers of $Q_{\Lambda}$.

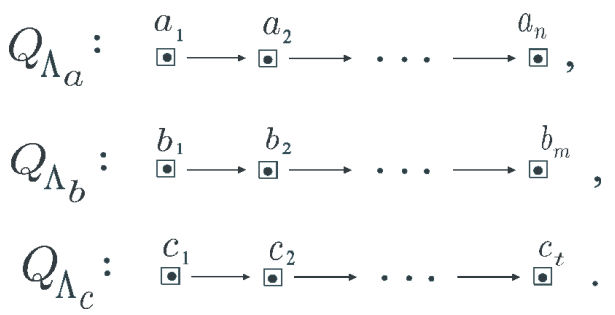

In case one of the numbers $n, m$ or $t$ is zero then the corresponding quiver $Q_{\Lambda_{a}}, Q_{\Lambda_{b}}$ or $Q_{\Lambda_{c}}$ is the empty set. We set $w(\Lambda):=\left|\left(Q_{\Lambda_{w}}\right)_{0}\right|$ for $w \in\{a, b, c\}$ where $|X|$ stands for the cardinality of a given set $X$.

Definition 5.2. For a trivial extension $\Lambda$ of Class II, we fix the universal covering $\pi: \mathbb{Z} \mathbf{D}_{N} \rightarrow{ }_{S} \Gamma_{\Lambda}$ such that $h_{\mathbb{Z} \mathbf{D}_{N}}\left(\pi^{-1}\left(r P_{3}\right)\right)=N-1$; and moreover, in case $N=4$, we add the condition $h_{\mathbb{Z} \mathbf{D}_{4}}\left(\pi^{-1}\left(r P_{a_{1}}\right)\right)=$ 1 ( see 2.11 (a)). Consider a lifting ( see $[8,3.5]$ ) of ${ }_{S} \Gamma_{\Lambda}$ to $\mathbb{Z} \mathbf{D}_{N}$ at $r P_{3}$. For each $w \in\{a, b, c\}$, we define the set $\operatorname{Ins}\left(Q_{\Lambda_{w}}\right)$ as the subset of the bottom border of $\mathbb{Z} \mathbf{D}_{N}$ which is illustrated in the following picture. 


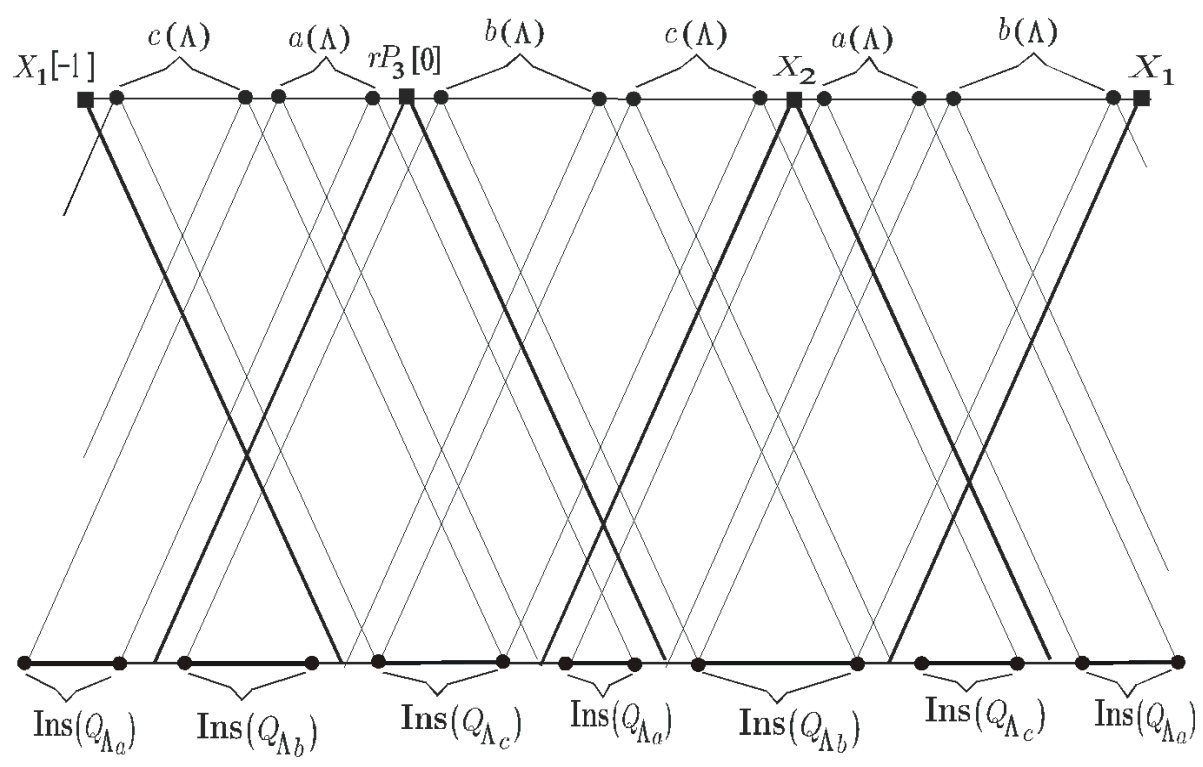

In what follows, we prove that the radicals of the projective modules associated to the insertion vertices of $Q_{\Lambda}$ belonging to $Q_{\Lambda_{w}}$, for $w \in$ $\{a, b, c\}$, lift to $\operatorname{Ins}\left(Q_{\Lambda_{w}}\right)$.

Proposition 5.3. Let $\Lambda$ be a trivial extension of Class II and Cartan class $\mathbf{D}_{N}$. Consider a lifting of ${ }_{S} \Gamma_{\Lambda}$ to $\mathbb{Z} \mathbf{D}_{N}$ as in 5.2.

(a) Let $x$ be an insertion vertex of $Q_{\Lambda}$ and $w \in\{a, b, c\}$. If $x \in$ $Q_{\Lambda_{w}}$ then $\pi^{-1}\left(r_{\Lambda} P_{x}\right) \subseteq \operatorname{Ins}\left(Q_{\Lambda_{w}}\right)$.

(b) The radicals $r P_{1}, r P_{2}$ and $r P_{3}$ lift to $\mathbb{Z} \mathbf{D}_{N}$ as is indicated in the following picture, where

$$
\begin{aligned}
& r P_{2}[0]= \begin{cases}X_{2} & \text { if } c(\Lambda)+b(\Lambda) \text { is even, } \\
Y_{2} & \text { if } c(\Lambda)+b(\Lambda) \text { is odd. }\end{cases} \\
& r P_{1}[0]= \begin{cases}X_{1} & \text { if } c(\Lambda)+a(\Lambda) \text { is even, } \\
Y_{1} & \text { if } c(\Lambda)+a(\Lambda) \text { is odd. }\end{cases}
\end{aligned}
$$




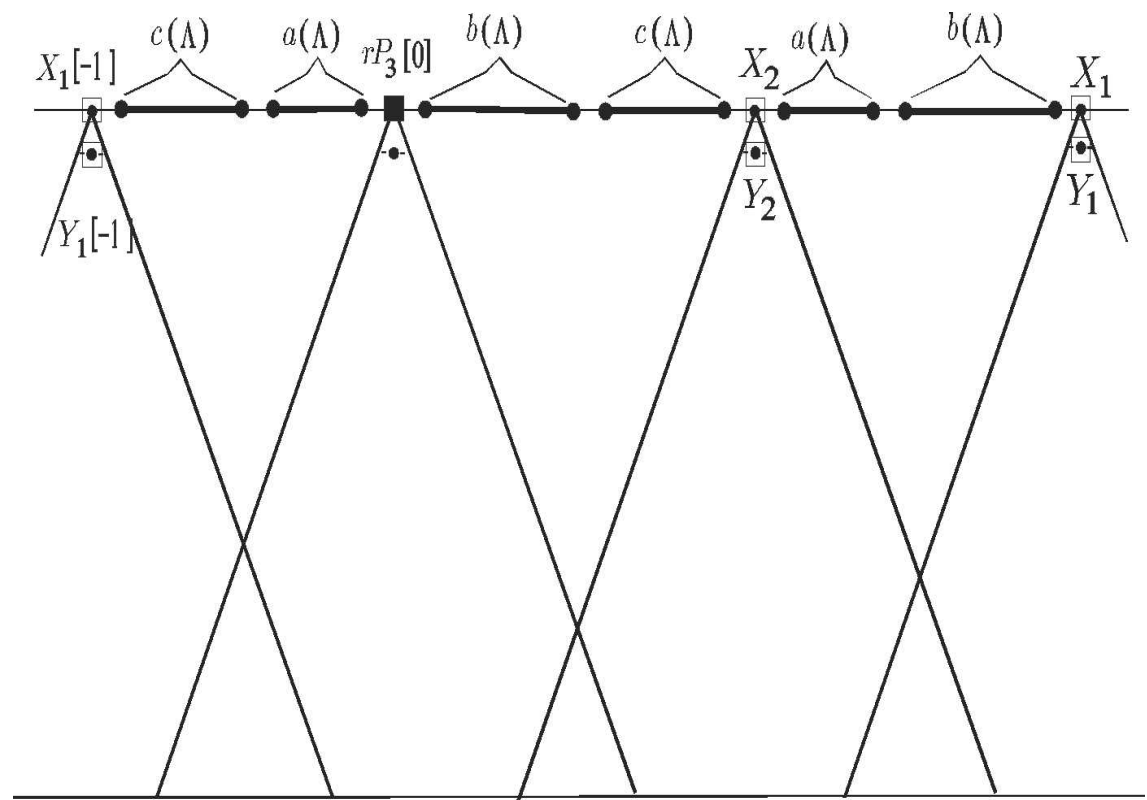

Proof. We will proceed by induction on the number of elemental cycles of $Q_{\Lambda}$ (see 1.4). If there are not elimination cycles in $Q_{\Lambda}$ (see 2.1 ), then $\Lambda$ is minimal and so $a(\Lambda)=n, b(\Lambda)=m$ and $c(\Lambda)=t$. Hence, in this case, the result follows from 2.11 (a) and 2.14.

Suppose there is at least one elimination cycle $C$ in the ordinary quiver $Q_{\Lambda}$. Since $C \subseteq \cup_{w \in\{a, b, c\}} Q_{\Lambda_{w}}$, we may assume that $C$ is contained in $Q_{\Lambda_{c}}$ (for the other cases, the proof is quite similar). Let $C=z \leftarrow z_{1} \leftarrow z_{2} \leftarrow \cdots \leftarrow z_{m-1} \leftarrow z$. Consider the trivial extension $\Gamma$ of Cartan class $\mathbf{D}_{N-m+1}$ obtained from $\Lambda$ by eliminating the cycle $C$. So, by 4.11 , we have the full and faithful functor

$$
\Phi:=\Phi_{z, 3}: k\left(\mathbb{Z} \mathbf{D}_{N-m+1}\right) \rightarrow k\left(\mathbb{Z} \mathbf{D}_{N}\right) .
$$

By induction, we have that the result is true for $\Gamma$. Furthermore, since $z \in Q_{\Gamma_{c}}$ is an insertion vertex of $Q_{\Gamma}$, it follows that $r_{\Gamma} P_{z}[0] \in$ $\operatorname{Ins}\left(Q_{\Gamma_{c}}\right)$. Now, consider the partition $\left\{C_{r_{\Gamma} P_{z}[0]}^{-}[i], C_{r_{\Gamma} P_{z}[0]}^{+}[i]: i \in \mathbb{Z}\right\}$ of $\mathbb{Z} \mathbf{D}_{N-m+1}$ (see 4.2). In the following picture, we illustrate this partition where the shaded regions correspond to $C_{r_{\Gamma} P_{z}[0]}^{-}$. 


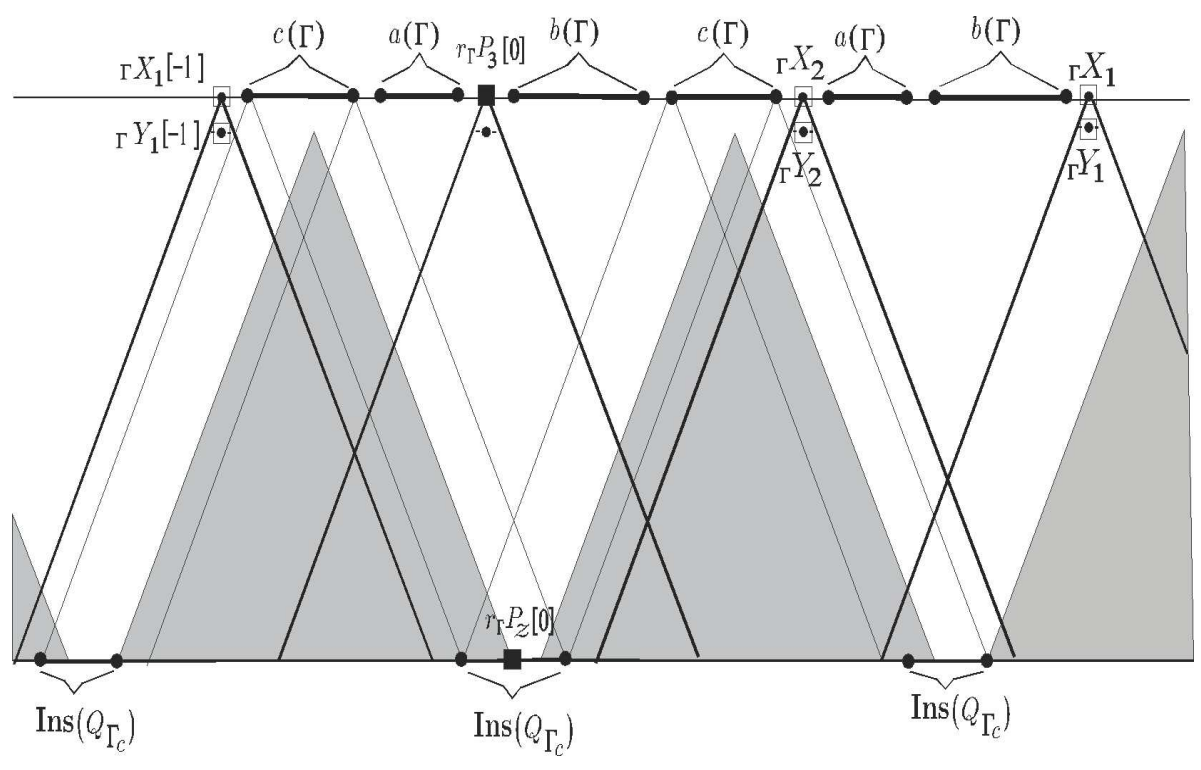

$\Phi$ changes the partition $\left\{C_{r_{\Gamma} P_{z}[0]}^{-}[i], C_{r_{\Gamma} P_{z}[0]}^{+}[i]: i \in \mathbf{Z}\right\}$ of $\mathbb{Z} \mathbf{D}_{N-m+1}$ as is indicated in the following picture (see 4.9 and 4.10). 


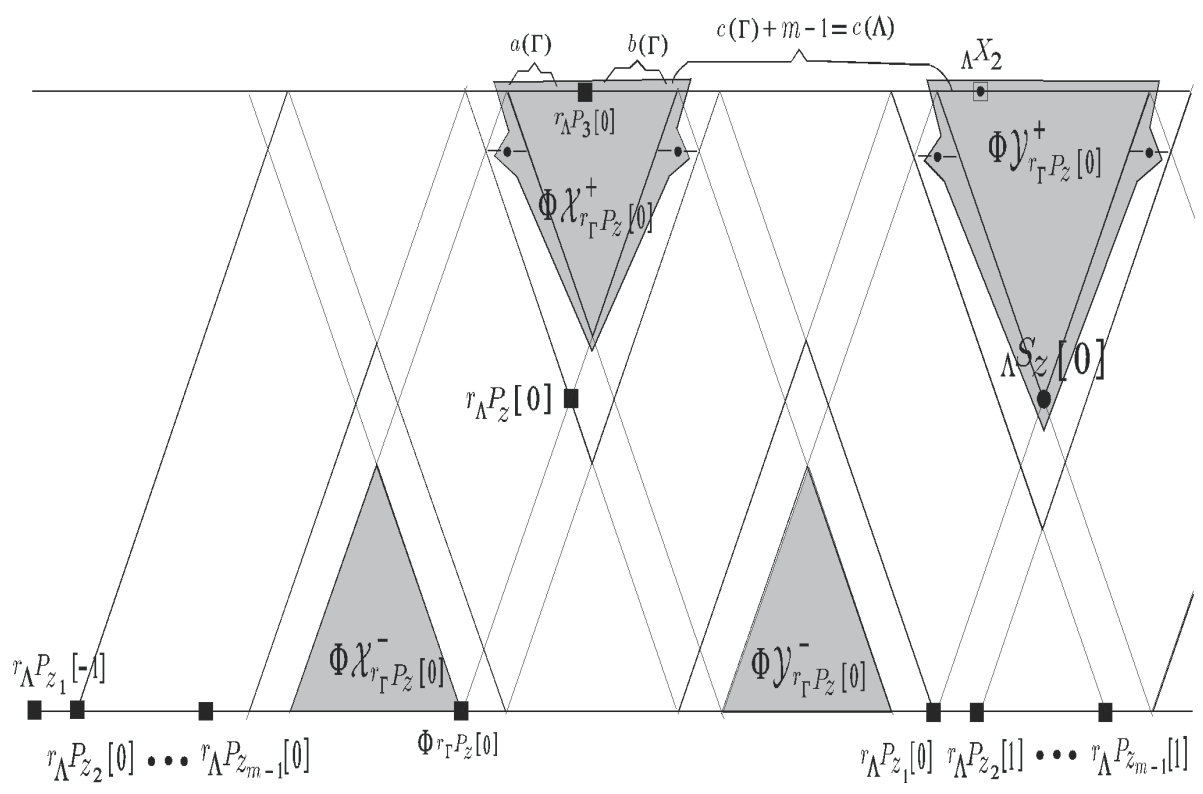

Moreover, by 4.11 (b), we have that $\Phi\left(r_{\Gamma} P_{t}[0]\right)=r_{\Lambda} P_{t}[0]$ for any $t \in\left(Q_{\Gamma}\right)_{0}$ with $t \neq z$. Now, we are ready to prove items (a) and (b).

(a) Let $x$ be an insertion vertex in $Q_{\Lambda}$. To prove (a), it is enough tho consider two cases: (a1) $x \in Q_{\Lambda_{a}}$ and (a2) $x \in Q_{\Lambda_{c}}$ (the case $x \in Q_{\Lambda_{b}}$ is quite similar to the first one).

(a1) Let $x \in Q_{\Lambda_{a}}$. Since $Q_{\Lambda_{a}}=Q_{\Gamma_{a}}$, we get by induction that $r_{\Gamma} P_{x}[0] \in \operatorname{Ins}\left(Q_{\Gamma_{a}}\right)$. Moreover, by the two figures above, it follows that $\Phi\left(\operatorname{Ins}\left(Q_{\Gamma_{a}}\right)\right)=\operatorname{Ins}\left(Q_{\Lambda_{a}}\right)$. Hence $r_{\Lambda} P_{x}[0]=\Phi\left(r_{\Gamma} P_{x}[0]\right) \in \Phi\left(\operatorname{Ins}\left(Q_{\Gamma_{a}}\right)\right)=$ $\operatorname{Ins}\left(Q_{\Lambda_{a}}\right)$; proving (a) in this case.

(a2) Let $x \in Q_{\Lambda_{c}}$. Then, either $x \in\left(Q_{\Gamma_{c}}\right)_{0} \backslash\{z\}$ or $x \in\left\{z_{1}, z_{2}, \cdots, z_{m-1}\right\}$. If $x \in\left\{z_{1}, z_{2}, \cdots, z_{m-1}\right\}$, by the two figures above, we get that $r_{\Lambda} P_{z_{i}}[0] \in$ $\operatorname{Ins}\left(Q_{\Lambda_{c}}\right)$ for any $i=1,2, \cdots, m-1$. Thus $r_{\Lambda} P_{x}[0] \in \operatorname{Ins}\left(Q_{\Lambda_{c}}\right)$.

In case $x \in\left(Q_{\Gamma_{c}}\right)_{0} \backslash\{z\}$, by induction, we obtain that $r_{\Gamma} P_{x}[0] \in$ Ins $\left(Q_{\Gamma_{c}}\right)$. On the other hand, using the two figures above, we get that $\Phi\left(\operatorname{Ins}\left(Q_{\Gamma_{c}}\right)\right) \subseteq \operatorname{Ins}\left(Q_{\Lambda_{c}}\right)$. Hence $r_{\Lambda} P_{x}[0]=\Phi\left(r_{\Gamma} P_{x}[0]\right) \in \Phi\left(\operatorname{Ins}\left(Q_{\Gamma_{c}}\right)\right) \subseteq$ $\operatorname{Ins}\left(Q_{\Lambda_{c}}\right)$; proving (a). 
(b) Since by assumption $C \subseteq Q_{\Lambda_{c}}$, we get that $Q_{\Lambda_{a}}=Q_{\Gamma_{a}}$ and $Q_{\Lambda_{b}}=Q_{\Gamma_{b}}$. Therefore $a(\Lambda)=a(\Gamma)$ and $b(\Lambda)=b(\Gamma)$. On the other hand $c(\Lambda)=c(\Gamma)+m-1$. We only check the first equality in (b) since the second one can be checked in a very similar way.

We may assume that $c(\Gamma)+b(\Gamma)$ is even ( the other case is similar). Then, by induction, we have that $r_{\Gamma} P_{2}[0]={ }_{\Gamma} X_{2}$. Furthermore, by 4.11 (b), we obtain that $r_{\Lambda} P_{2}[0]=\Phi\left(r_{\Gamma} P_{2}[0]\right)=\Phi\left({ }_{\Gamma} X_{2}\right)$. Now, since $r_{\Gamma} P_{3}[0] \in \mathcal{X}_{r_{\Gamma} P_{z}[0]}^{+}$, we have that $\Phi=\Phi_{m-1, r_{\Gamma} P_{z}[0], \mathcal{X}^{+}}$(see in 4.6). Moreover, we have that ${ }_{\Gamma} X_{2} \in \mathcal{Y}_{r_{\Gamma} P_{z}[0]}^{+}$(see the picture above); and hence, by the definition of $\Phi$, it follows that

(*) $\quad r_{\Lambda} P_{2}[0]=\Phi\left({ }_{\Gamma} X_{2}\right)=\sigma^{m-1}\left({ }_{\Lambda} X_{2}\right)= \begin{cases}{ }_{\Lambda} X_{2} & \text { if } m-1 \text { is even } \\ { }_{\Lambda} Y_{2} & \text { if } m-1 \text { is odd. }\end{cases}$

Since $c(\Lambda)=c(\Gamma)+m-1$, it follows that $c(\Lambda)+b(\Lambda)=c(\Gamma)+b(\Gamma)+$ $m-1$. As a consequence, we have that $c(\Lambda)+b(\Lambda)$ and $m-1$ have the same parity since $c(\Gamma)+b(\Gamma)$ is even. Hence, from $(*)$, we get the first equality in (b).

\section{Construction of the configuration associated to a triv- ial extension of Cartan class $\mathbf{D}_{N}$.}

Let $\Lambda$ be a trivial extension of Cartan class $\mathbf{D}_{N}$. The ordinary quiver $Q_{\Lambda}$, of a trivial extension $\Lambda$ is described in 1.2 and 1.9. We recall that the quiver $Q_{\Lambda}$ can be described by drawing only the minimal quiver $Q_{\Lambda_{\min }}$ of $\Lambda_{\min }$ (see 1.8).

Consider the universal covering $\pi: \mathbb{Z} \mathbf{D}_{N} \rightarrow{ }_{S} \Gamma_{\Lambda}$ of ${ }_{S} \Gamma_{\Lambda}$. In this section, we give an algorithm to determine the configuration $\widetilde{\mathcal{C}_{\Lambda}}$ of $\mathbb{Z} \mathbf{D}_{N}$ associated to $\Lambda$. We recall that $\widetilde{\mathcal{C}_{\Lambda}}:=\pi^{-1}\left(\mathcal{C}_{\Lambda}\right)$, where $\mathcal{C}_{\Lambda}$ is the set of vertices of ${ }_{S} \Gamma_{\Lambda}$ representing the radicals of the indecomposable projective $\Lambda$-modules (see $[8,2.7])$. We define the subset $r \mathcal{P}\left(\Lambda, \mathbb{Z} \mathbf{D}_{N}\right)$ of $\left(\mathbb{Z} \mathbf{D}_{N}\right)_{0}$ and prove that $r \mathcal{P}\left(\Lambda, \mathbb{Z} \mathbf{D}_{N}\right)[\mathbb{Z}]:=\cup_{i \in \mathbb{Z}} r \mathcal{P}\left(\Lambda, \mathbb{Z} \mathbf{D}_{N}\right)[i]$ is the desired configuration.

We start by defining some notations needed to state the algorithm: the sections $\mathcal{S}_{x}^{+}$and $\mathcal{S}_{x}^{-}$, the initial vertices, and the functions border and height defined on the set of vertices of $Q_{\Lambda}$. The algorithm is stated 
in the Theorem 6.8 and illustrated in the Example 6.3. The remainder of the section is devoted to the proof of the Theorem 6.8 .

In what follows, we introduce two sections in $\mathbb{Z} \mathbf{D}_{N}$ which are associated with a given vertex in $\mathbb{Z} \mathbf{D}_{N}$. These sections will play an important role in the algorithm to compute the configuration associated to the trivial extension $\Lambda$.

Definition 6.1. Associated with a given vertex $(p, q)$ in $\mathbb{Z D}_{N}$, we have the following two sections in $\mathbb{Z} \mathbf{D}_{N}$ :

$$
\begin{gathered}
\mathcal{S}_{(p, q)}^{+}:=\{(p+N-1, i): 1 \leq i \leq N-1\} ; \\
\mathcal{S}_{(p, q)}^{-}:=\left\{\begin{array}{lc}
\{(p+q, i): 1 \leq i \leq N-1\} & \text { if } q \neq N, \\
\mathcal{S}_{(p, N)}^{+} & \text {if } q=N .
\end{array}\right.
\end{gathered}
$$

In the following picture, we illustrate the shape of the two parallel sections $\mathcal{S}_{x}^{+}$and $\mathcal{S}_{x}^{-}$associated with the vertex $x=(p, q)$.

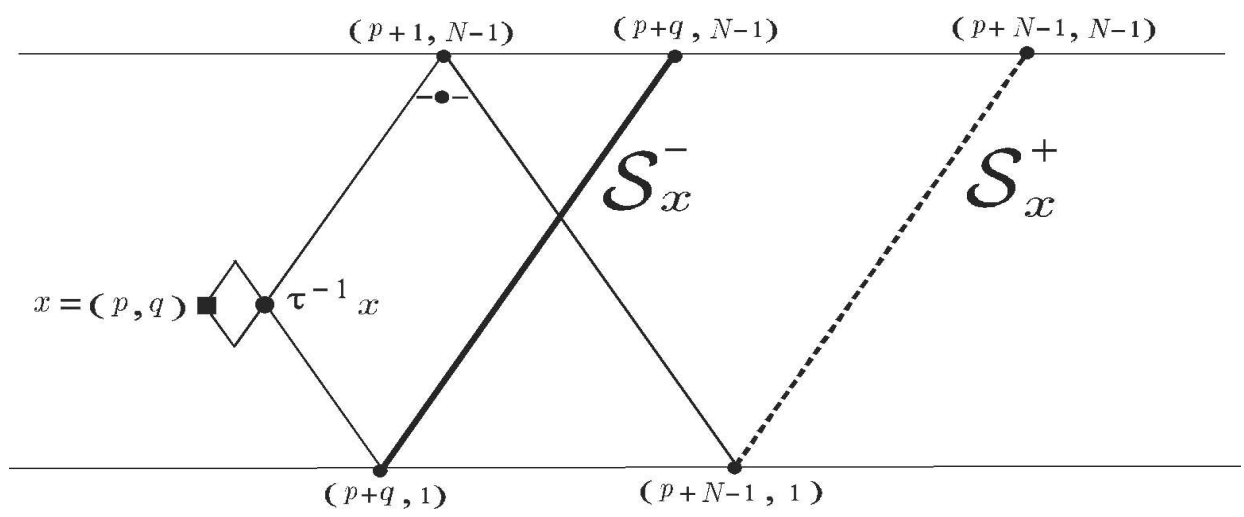

Definition 6.2. Let $\Lambda$ be a trivial extension of Cartan class $\mathbf{D}_{N}$. The initial vertices $\operatorname{Ini}(\Lambda)$ of $Q_{\Lambda}$ are those in the set

$$
\operatorname{Ini}(\Lambda):= \begin{cases}\{1,4\} & \text { if } \Lambda \text { is of Class } I, \\ \{1,2,3\} & \text { otherwise. }\end{cases}
$$

The elements of the set $\left(Q_{\Lambda}\right)_{0} \backslash \operatorname{Ini}(\Lambda)$ are called the non-initial vertices of $Q_{\Lambda}$. 
Definition 6.3. Let $\Lambda$ be a trivial extension of Cartan class $\mathbf{D}_{N}$. Any non-initial vertex $i$ of $Q_{\Lambda}$ induces two full connected subquivers of $Q_{\Lambda}:$ namely, $Q_{\Lambda}^{-, i}$ and $Q_{\Lambda}^{+, i}$ which are defined as follows.

(a) $Q_{\Lambda}^{-, i} \cap Q_{\Lambda}^{+, i}=\{i\}$ and $Q_{\Lambda}^{-, i} \cup Q_{\Lambda}^{+, i}=Q_{\Lambda}$;

(b) $Q_{\Lambda}^{-, i}=Q_{\Lambda^{\prime}}$ where $\Lambda^{\prime}$ is a trivial extension of Cartan class $\mathbf{D}_{N^{\prime}}$

(c) if $i$ is an insertion vertex of $Q_{\Lambda}$, we set $Q_{\Lambda}^{+, i}:=\{i\}$; and otherwise, $Q_{\Lambda}^{+, i}=Q_{\Lambda^{\prime \prime}}$ where $\Lambda^{\prime \prime}$ is a trivial extension of Cartan class $\mathbf{A}_{n^{\prime \prime}}$ with $n^{\prime \prime}>1$.

The quivers $Q_{\Lambda}^{-, i}$ and $Q_{\Lambda}^{+, i}$ are said to be the splitting of $Q_{\Lambda}$ at the vertex $i$.

Example 6.1. Let $\Lambda$ be the trivial extension of Class I and Cartan class $D_{6}$ given by the quiver:

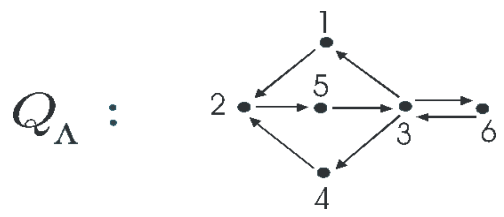

The splitting of $Q_{\Lambda}$ at the vertex $i=3$ is

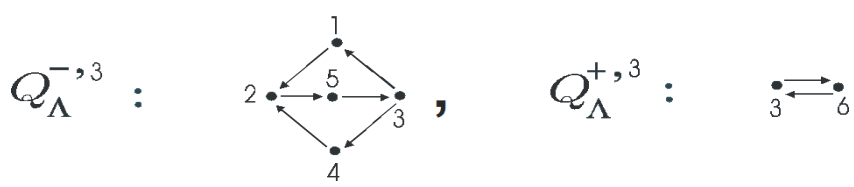

Definition 6.4. Let $\Lambda$ be a trivial extension of Cartan class $\mathbf{D}_{N}$. The border map $\partial_{\Lambda}:\left(Q_{\Lambda}\right)_{0} \rightarrow\{-, *,+\}$ is defined as follows:

(a) $\partial_{\Lambda}(i):=*$ for any initial vertex $i$ of $Q_{\Lambda}$;

(b) for any non-initial vertex $j \in Q_{\Lambda_{\text {min }}}$, we set $\partial_{\Lambda}(j):=+$ if there is an arrow $j \rightarrow v$ with $v \in \operatorname{Ini}(\Lambda)$, otherwise $\partial_{\Lambda}(j):=-$; 
(c) let $C$ and $C^{\prime}$ be two elemental cycles of $Q_{\Lambda}$ (see 1.4) meeting only at the non-initial vertex $x$. Suppose we already have defined $\partial_{\Lambda}$ on the vertices of the cycle $C$; so, for any vertex $z$ of $C^{\prime}$, different from $x$, we set $\partial_{\Lambda}(z):=+$ if there is an arrow $z \rightarrow x$, otherwise $\partial_{\Lambda}(z):=-$.

Definition 6.5. Let $\Lambda$ be a trivial extension of Cartan class $\mathbf{D}_{N}$. The height function $h_{\Lambda}:\left(Q_{\Lambda}\right)_{0} \rightarrow \mathbb{N}$ is defined as follows.

(a) For the initial vertices of $Q_{\Lambda}$ :

(a1) if $\Lambda$ is of Class I, we set $h_{\Lambda}(1):=N-1$ and $h_{\Lambda}(4):=N$;

(a2) if $\Lambda$ is of Class II, we set $h_{\Lambda}(3):=N-1$ and

$$
\begin{aligned}
& h_{\Lambda}(2):= \begin{cases}N-1 & \text { if } c(\Lambda)+b(\Lambda) \text { is even }, \\
N & \text { if } c(\Lambda)+b(\Lambda) \text { is odd } ;\end{cases} \\
& h_{\Lambda}(1):= \begin{cases}N-1 & \text { if } c(\Lambda)+a(\Lambda) \text { is even, } \\
N & \text { if } c(\Lambda)+a(\Lambda) \text { is odd; }\end{cases}
\end{aligned}
$$

where $a(\Lambda), b(\Lambda)$ and $c(\Lambda)$ are the numbers defined in 5.1

(a3) if $\Lambda$ is of Class III, we set $h_{\Lambda}(1):=N-2, h_{\Lambda}(3):=N-1$ and $h_{\Lambda}(2):=N$.

(b) $h_{\Lambda}(i):=\left|\left(Q_{\Lambda}^{+, i}\right)_{0}\right|$ for any non-initial vertex $i$ of $Q_{\Lambda}$.

Remark 6.6. Let $\Lambda$ be a trivial extension of Cartan class $\mathbf{D}_{N}$. Consider the universal covering $\pi: \mathbb{Z} \mathbf{D}_{N} \rightarrow{ }_{S} \Gamma_{\Lambda}$ and a lifting of ${ }_{S} \Gamma_{\Lambda}[0]$ to $\mathbb{Z} \mathbf{D}_{N}$ as in 2.10 (c), 2.11, 2.12 and 5.3. Then, for any initial vertex $i$ of $Q_{\Lambda}$, we have that $h_{\Lambda}(i)=h_{\mathbb{Z} \mathbf{D}_{N}}\left(r P_{i}[0]\right)$.

Example 6.2. Let $\Lambda$ be the trivial extension of Class I and Cartan class $\mathbf{D}_{9}$ given by the quiver

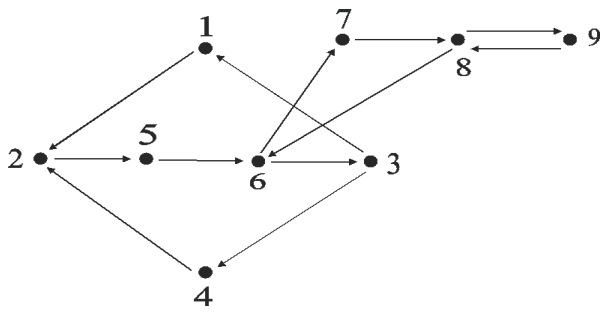


In the following table, we compute the values of the height function and the border map associated to $\Lambda$.

\begin{tabular}{|r|c|c|c|c|c|c|c|c|c|}
\hline$i \in\left(Q_{\Lambda}\right)_{0}$ & 1 & 2 & 3 & 4 & 5 & 6 & 7 & 8 & 9 \\
\hline$\partial_{\Lambda}(i)$ & $*$ & - & + & $*$ & - & - & - & + & + \\
\hline$h_{\Lambda}(i)$ & 8 & 1 & 1 & 9 & 1 & 4 & 1 & 2 & 1 \\
\hline
\end{tabular}

Definition 6.7. Let $\Lambda$ be a trivial extension of Cartan class $\mathcal{D}_{N}$ and $u$ a vertex of the top border of $\mathbb{Z} \mathbf{D}_{N}$. Consider the universal covering $\pi: \mathbb{Z} \mathbf{D}_{N} \rightarrow{ }_{S} \Gamma_{\Lambda}$ and a lifting of ${ }_{S} \Gamma_{\Lambda}[0]$ to $\mathbb{Z} \mathbf{D}_{N}$ as in 2.10 (c), 2.11, 2.12 and 5.3. We define the set $r \mathcal{P}\left(\Lambda, \mathbb{Z} \mathbf{D}_{N}\right):=\left\{\widehat{r P}_{i} \in\left(\mathbb{Z D}_{N}\right)_{0}: i \in\right.$ $\left.\left(Q_{\Lambda}\right)_{0}\right\}$ by the following rules.

(a) For the initial vertices of $Q_{\Lambda}$ :

(a1) if $\Lambda$ is of Class I, we set $\widehat{r P_{1}}:=u$ and define $\widehat{r P_{4}}$ as is indicated in the picture below

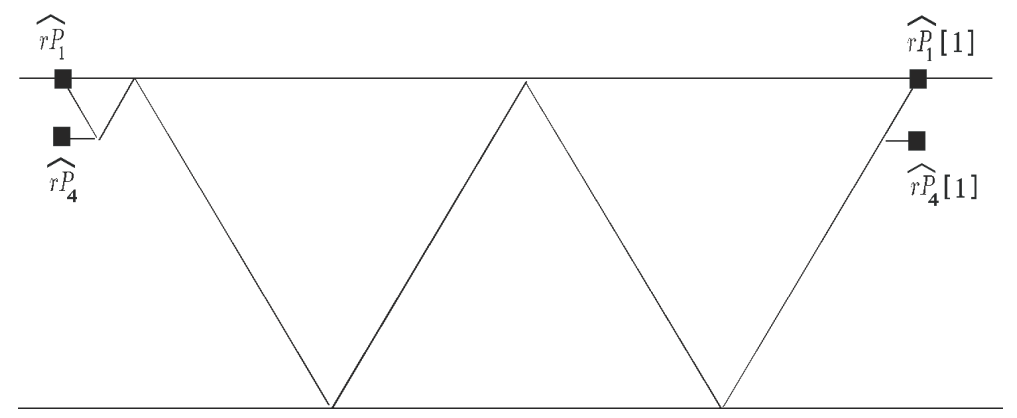

(a2) if $\Lambda$ is of Class II, we set $\widehat{r P_{3}}:=u$ and define $\widehat{r P}_{i}$, for $i=1,2$, as is indicated in the picture below, where

$$
\begin{aligned}
& \widehat{r P_{2}}:= \begin{cases}X_{2} & \text { if } c(\Lambda)+b(\Lambda) \text { is even, } \\
Y_{2} & \text { if } c(\Lambda)+b(\Lambda) \text { is odd. }\end{cases} \\
& \widehat{r P_{1}}:= \begin{cases}X_{1} & \text { if } c(\Lambda)+a(\Lambda) \text { is even, } \\
Y_{1} & \text { if } c(\Lambda)+a(\Lambda) \text { is odd. }\end{cases}
\end{aligned}
$$




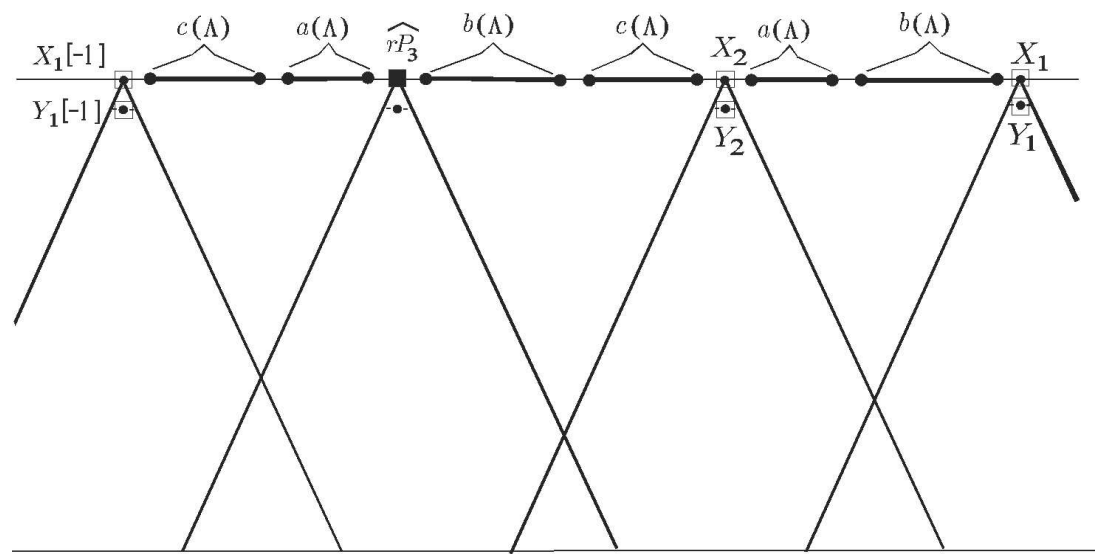

(a3) if $\Lambda$ is of Class III, we set $\widehat{r P_{3}}:=u$ and define $\widehat{r P}_{i}$, for $i=1,2$, as is indicated in the picture below

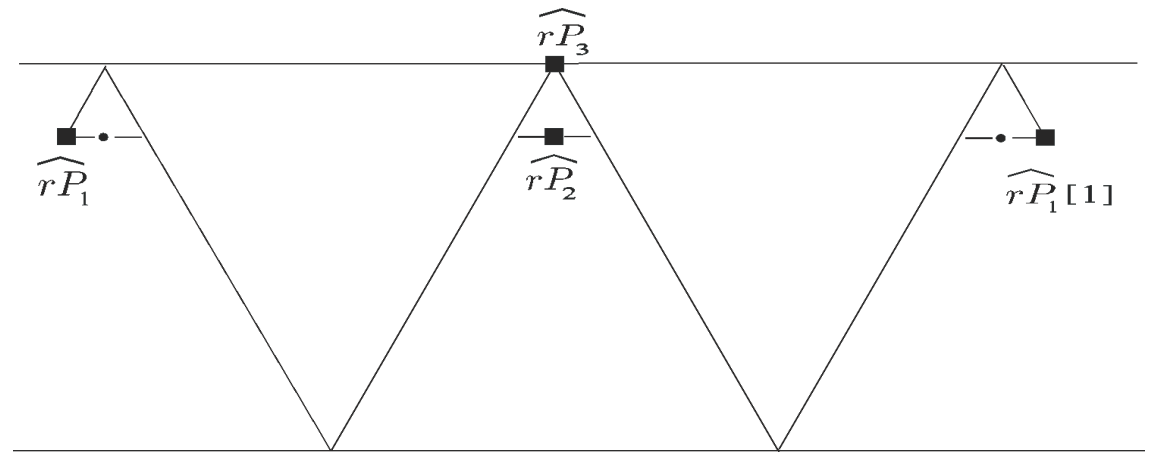

(b) Let $i \rightarrow j$ be an arrow in $Q_{\Lambda}$ with $i$ a non-initial vertex. Suppose that we already have defined $\widehat{r P_{j}}$. Then, we set $\widehat{r P}_{i}:=X_{i}$ for the vertex $X_{i}$ in the section $\mathcal{S}_{\overrightarrow{r P_{j}}}^{\partial_{\Lambda}(i)}$ satisfying the equality $h_{\mathbb{Z} \mathbf{D}_{N}}\left(X_{i}\right)=h_{\Lambda}(i)$.

Example 6.3. Let $\Lambda$ be the trivial extension of Class II and Cartan class $\mathbf{D}_{9}$ given by the quiver 


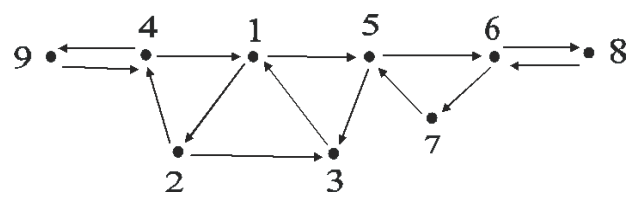

In the following table, we compute the values of the height function and the border map associated to $\Lambda$.

\begin{tabular}{|r|c|c|c|c|c|c|c|c|c|}
\hline$i \in\left(Q_{\Lambda}\right)_{0}$ & 1 & 2 & 3 & 4 & 5 & 6 & 7 & 8 & 9 \\
\hline$\partial_{\Lambda}(i)$ & $*$ & $*$ & $*$ & + & + & - & + & + & + \\
\hline$h_{\Lambda}(i)$ & 8 & 8 & 8 & 2 & 4 & 2 & 1 & 1 & 1 \\
\hline
\end{tabular}

In the picture below, we indicate the vertices of $r \mathcal{P}\left(\Lambda, \mathbb{Z} \mathbf{D}_{9}\right)$ with small black squares

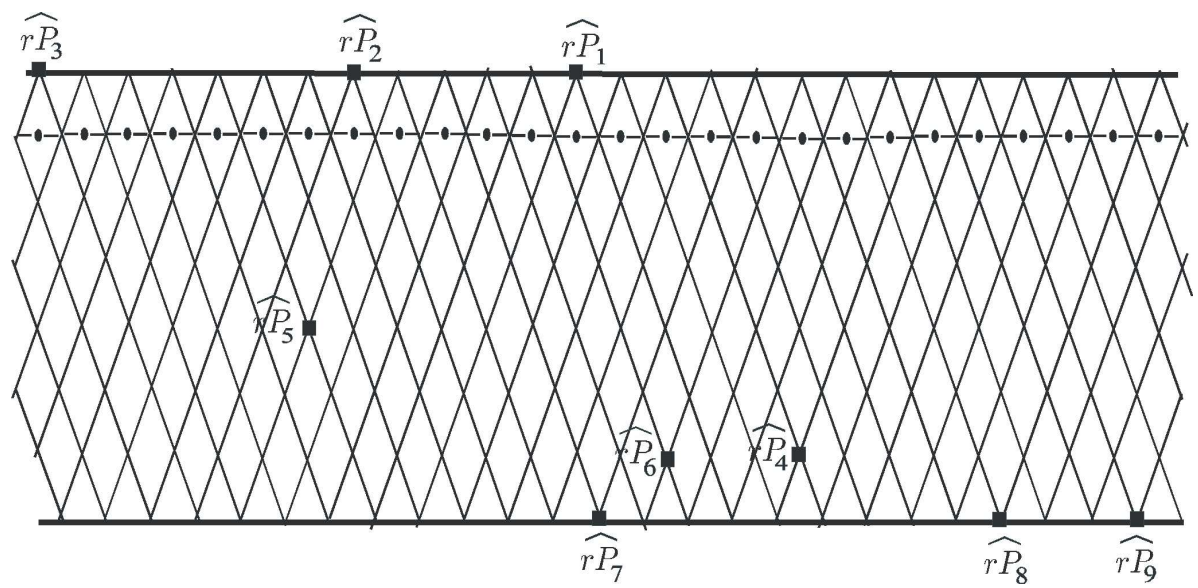

Let $r \mathcal{P}\left(\Lambda, \mathbb{Z} \mathbf{D}_{N}\right)[\mathbb{Z}]:=\left\{\tau^{s(2 N-3)}(x): x \in r \mathcal{P}\left(\Lambda, \mathbb{Z} \mathbf{D}_{N}\right), s \in \mathbb{Z}\right\}$. In what follows, we prove that such a set is the configuration $\widetilde{\mathcal{C}_{\Lambda}}$ of $\mathbb{Z} \mathbf{D}_{N}$ associated to $\Lambda$. This will be the main result of the paper. 
Theorem 6.8. Let $\Lambda$ be a trivial extension of Cartan class $\mathbf{D}_{N}$. Fix a vertex $u$ in $\mathbb{Z D}_{N}$ and take $i_{0} \in Q_{\Lambda}$ with $i_{0}:=1$ if $\Lambda$ is of Class $I$ and $i_{0}:=3$ otherwise. Consider the universal covering $\pi: \mathbb{Z}_{N} \rightarrow{ }_{S} \Gamma_{\Lambda}$ and a lifting of ${ }_{S} \Gamma_{\Lambda}[0]$ to $\mathbb{Z} \mathbf{D}_{N}$ as in 2.10 (c), 2.11, 2.12, 5.3 and such that $r P_{i_{0}}[0]=u$. Let $r \mathcal{P}\left(\Lambda, \mathbb{Z} \mathbf{D}_{N}\right)$ be the set associated to these data (see 6.7). Then

(a) $\widetilde{\mathcal{C}_{\Lambda}}=r \mathcal{P}\left(\Lambda, \mathbb{Z} \mathbf{D}_{N}\right)[\mathbb{Z}] ;$

(b) $\pi\left(\widehat{r P}_{i}\right)=r P_{i}$ for any vertex $i \in Q_{\Lambda}$;

(c) $h_{\mathbb{Z} \mathbf{D}_{N}}\left(r P_{i}[0]\right)=h_{\Lambda}(i)$ for any vertex $i \in Q_{\Lambda}$.

The proof of 6.8 will be carried out by induction on the number of minimal oriented cycles of $Q_{\Lambda}$. To do that, we delete an elemental cycle $C$ (see 1.4) of $Q_{\Lambda}$ obtaining a trivial extension $\Gamma$ having less elemental cycles than $\Lambda$. So, we get the functions: $h_{\Lambda}, h_{\Gamma}, \partial_{\Lambda}, \partial_{\Gamma}$. In order to prove that theorem, we have to stablish, firstly, the relationship between such functions. To start with, it is easy to see that the restriction of $\partial_{\Lambda}$ to $\left(Q_{\Gamma}\right)_{0}$ is $\partial_{\Gamma}$. However, the relationship between $h_{\Lambda}$ and $h_{\Gamma}$ is more complicated. Hence, to solve this problem, we introduce the notions of $C$-linked and $C$-free vertices of $Q_{\Lambda}$.

Definition 6.9. Let $\Lambda$ be a trivial extension of Cartan class $\mathbf{D}_{N}$, and $C$ be an elemental cycle of $Q_{\Lambda}$ which is not contained in $Q_{\Lambda_{\min }}$. It can be seen easily, that there exists only one chain $C=C_{1}, C_{2}, \cdots, C_{\ell}$ consisting of elemental cycles of $Q_{\Lambda}$ such that: (a) $\left(C_{i}\right)_{0} \cap\left(C_{i+1}\right)_{0}=$ $\left\{x_{i}\right\}$ where $x_{i}$ is a vertex in $Q_{\Lambda}$ for $1 \leq i<\ell$, (b) $C_{i} \nsubseteq Q_{\Lambda_{\text {min }}}$ for $1 \leq i<\ell$ and (c) $C_{\ell} \subseteq Q_{\Lambda_{\text {min }}}$. The vertices $x_{1}, x_{2}, \cdots, x_{\ell-1}$ are said to be $C$-linked; and the remaining ones of $Q_{\Lambda}$, which are non-initial vertices, will be called $C$-free.

Example 6.4. Consider the trivial extension $\Lambda$ of Class $I_{2}$ and Cartan class $\mathbf{D}_{9}$, having as quivers $Q_{\Lambda}$ and $Q_{\Lambda_{\text {min }}}$ the following ones
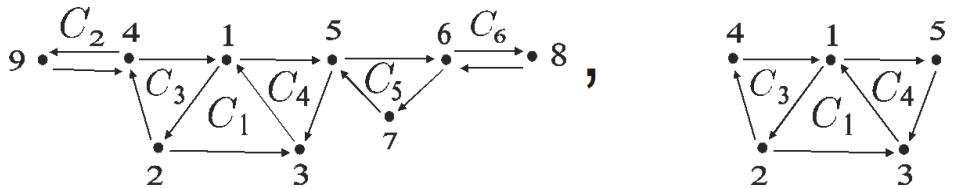
The elemental cycles of $Q_{\Lambda}$ are: $C_{1}, C_{2}, C_{3}, C_{4}, C_{5}$ and $C_{6}$; and can be seen in the above figures. In this case, we have

-) vertices $C_{2}-\left\{\begin{array}{l}\text { ligados: } 4 . \\ \text { libres: } 5,6,7,8,9 .\end{array} \bullet\right)$ vertices $C_{6}-\left\{\begin{array}{l}\text { ligados: } 5,6 \\ \text { libres: } 4,7,8,9 .\end{array}\right.$

Proposition 6.10. Let $\Lambda$ be a trivial extension of Cartan class $\mathbf{D}_{N}$ and $C$ an elimination cycle of $Q_{\Lambda}$ (see 2.1). Consider the trivial extension $\Gamma$ obtained from $\Lambda$ by eliminating the cycle $C$. Then, for any vertex $t \in Q_{\Gamma}$ which is a non-initial one, we have that

$$
h_{\Lambda}(t)= \begin{cases}h_{\Gamma}(t) & \text { if } t \text { is } C \text {-free }, \\ h_{\Gamma}(t)+\left|(\underline{C})_{0}\right|-1 & \text { if } t \text { is } C \text {-linked; }\end{cases}
$$

where $\left|(\underline{C})_{0}\right|$ is the number of vertices in the cycle $C$.

Proof. It is straightforward.

In the following proposition, we prove a result that will be essential to do the inductive step in the proof of 6.8. Here, we make use of the partition $\left\{C_{y}^{-}[i], C_{y}^{+}[i]: i \in \mathbb{Z}\right\}$ in $\mathbb{Z} \mathbf{D}_{N}$ induced by a vertex $y$ in the bottom border of $\mathbb{Z} \mathbf{D}_{N}$ (see 4.2 ).

Proposition 6.11. Let $\Lambda$ be a trivial extension of Cartan class $\mathbf{D}_{N}$. Consider the universal covering $\pi: \mathbb{Z}_{N} \rightarrow{ }_{S} \Gamma_{\Lambda}$ and a lifting of ${ }_{S} \Gamma_{\Lambda}[0]$ to $\mathbb{Z} \mathbf{D}_{N}$ as in 2.10 (c), 2.11, 2.12 and 5.3. Then, for any insertion vertex $z$ of $Q_{\Lambda}$ and any vertex $x \in Q_{\Lambda}$, the following statements hold.

(a) If $z \in Q_{\Lambda_{\min }}$ and $\partial_{\Lambda}(x) \neq *$, then $r P_{x}[0] \in C_{r P_{z}[0]}^{-}$.

(b) If $\mathcal{C}$ is an elemental cycle of $Q_{\Lambda}$ which is not contained in $Q_{\Lambda_{\text {min }}}$ and $z$ is a vertex in the cycle $\mathcal{C}$, then

$$
\begin{aligned}
& \text { (b1) } r P_{x}[0] \in C_{r P_{z}[0]}^{+} \text {if } x \text { is } \mathcal{C} \text {-linked, } \\
& \text { (b2) } r P_{x}[0] \in C_{r P_{z}[0]}^{-} \text {if } x \text { is } \mathcal{C} \text {-free. }
\end{aligned}
$$

Proof. We proceed by induction on the number of minimal cycles of $Q_{\Lambda}$.

(a) Let $z \in Q_{\Lambda_{\text {min }}}$ be an insertion vertex of $Q_{\Lambda}$ and $\partial_{\Lambda}(x) \neq *$. If $\Lambda$ is minimal, then (a) follows from 2.11, 2.12, 2.14 and 2.15. Suppose that $\Lambda$ is not minimal, and let $\mathcal{C}=x_{m} \leftarrow x_{1} \leftarrow x_{2} \leftarrow \cdots \leftarrow x_{m-1} \leftarrow x_{m}$ be an elimination cycle of $Q_{\Lambda}$. Consider the trivial extension $\Gamma$ of Cartan 
class $\mathbf{D}_{N-m+1}$ obtained from $\Lambda$ by eliminating the cycle $C$. We make use of 4.11, and so, we assume all the hypothesis needed there. Hence, we have a full an faithful functor $\Phi:=\Phi_{x_{m}, i_{0}}: k\left(\mathbb{Z} \mathbf{D}_{N-m+1}\right) \rightarrow$ $k\left(\mathbb{Z} \mathbf{D}_{N}\right)$. Thus, by 4.9 and 4.11 , we are able to compare the partition $\left\{C_{r_{\Gamma} P_{z}[0]}^{-}[i], C_{r_{\Gamma} P_{z}[0]}^{+}[i]: i \in \mathbb{Z}\right\}$ of $\mathbb{Z} \mathbf{D}_{N-m+1}$ with the partition $\left\{C_{r_{\Lambda} P_{z}[0]}^{-}[i], C_{r_{\Lambda} P_{z}[0]}^{+}[i]: i \in \mathbb{Z}\right\}$ of $\mathbb{Z} \mathbf{D}_{N}$ (see the following picture).

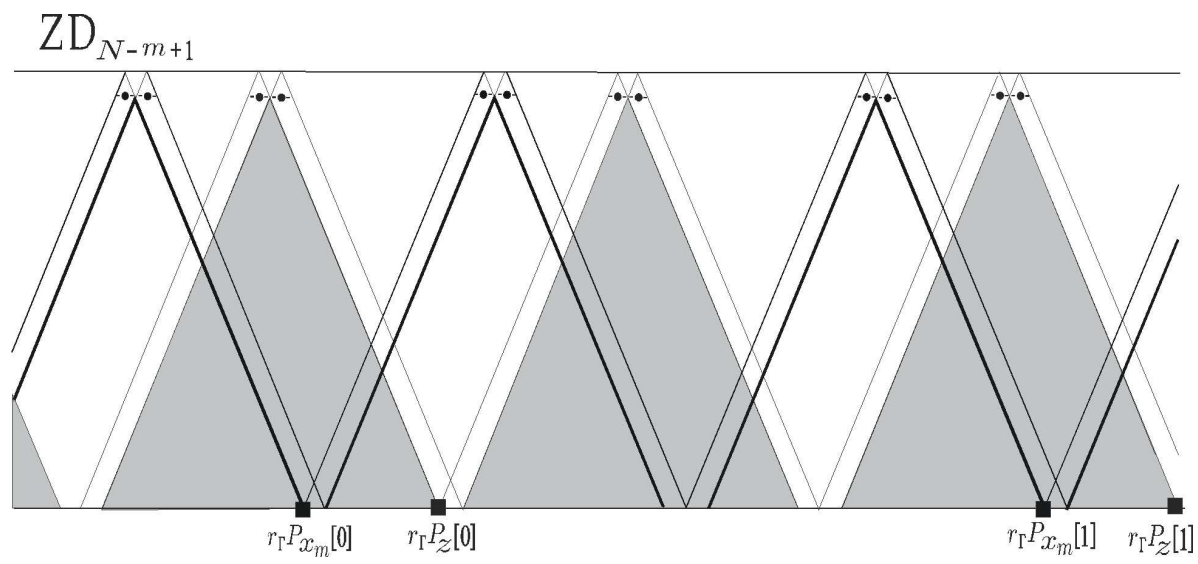

The shaded areas in the preceding picture correspond to $C_{r_{\Gamma} P_{z}[0]}^{-}$. Since $x_{m} \neq z$ we get by 4.11 (b1) that $\Phi\left(r_{\Gamma} P_{z}[0]\right)=r_{\Lambda} P_{z}[0]$. Furthermore, by definition of $\Phi$ and the above picture, it can be seen that $\Phi\left(C_{r_{\Gamma} P_{z}[0]}^{-}\right) \subseteq C_{\Phi\left(r_{\Gamma} P_{z}[0]\right)}^{-}$. Hence, we get

$$
\Phi\left(C_{r_{\Gamma} P_{z}[0]}^{-}\right) \subseteq C_{r_{\Lambda} P_{z}[0]}^{-} .
$$

If $x$ is a vertex in the cycle $\mathcal{C}$, then by the picture above, 3.1 (a) and 4.11, we obtain that: (i) $r_{\Lambda} P_{x_{m}}[0] \in C_{r_{\Lambda} P_{z}[0]}^{-}$and (ii) for $x \neq x_{m}$, the lifted radical $r_{\Lambda} P_{x}[0]$ is in the bottom border of $\mathbb{Z} \mathbf{D}_{N}$ and $r_{\Lambda} P_{x}[0] \notin$ $\Phi\left(C_{r_{\Gamma} P_{x_{m}}[0]}^{-}\right)$. Thus $r_{\Lambda} P_{x}[0] \in C_{r_{\Lambda} P_{z}[0]}^{-}$, proving (a) in this case.

Let, now, $x$ be a vertex in $Q_{\Gamma}$ different from $x_{m}$. Then by induction $r_{\Gamma} P_{x}[0] \in C_{r_{\Gamma} P_{z}[0]}^{-}$. Hence $r_{\Lambda} P_{x}[0]=\Phi\left(r_{\Gamma} P_{x}[0]\right) \in \Phi\left(C_{r_{\Gamma} P_{z}[0]}^{-}\right) \subseteq$ $C_{r_{\Lambda} P_{z}[0]}^{-}$by 4.11 (b) and (E1); proving (a).

(b) Let $\mathcal{C}$ be an elemental cycle of $Q_{\Lambda}$ which is not contained in $Q_{\Lambda_{\min }}$ and $z$ be a vertex in $\mathcal{C}$. In what follows, we analyze two possibilities. 
Case 1: Suppose that $\mathcal{C}$ is an elimination cycle of $Q_{\Lambda}$.

Let $\mathcal{C}=y \leftarrow y_{1} \leftarrow y_{2} \leftarrow \cdots \leftarrow y_{m-1} \leftarrow y$. By assumption, we have that $z \in\left\{y_{1}, y_{2}, \cdots, y_{m-1}\right\}$. Let $\Gamma$ be the trivial extension of Cartan class $\mathbf{D}_{N-m+1}$ obtained from $\Lambda$ by eliminating the cycle $\mathcal{C}$. We assume all the hypothesis needed in 4.11, and so, we have a full an faithful functor $\Phi:=\Phi_{y, i_{0}}: k\left(\mathbb{Z} \mathbf{D}_{N-m+1}\right) \rightarrow k\left(\mathbb{Z} \mathbf{D}_{N}\right)$. Consider the partition $\left\{C_{r_{\Gamma} P_{y}[0]}^{-}[i], C_{r_{\Gamma} P_{y}[0]}^{+}[i]: i \in \mathbb{Z}\right\}$ of $\mathbf{Z D}_{N-m+1}$, that can be illustrated by the shaded areas in the following picture.

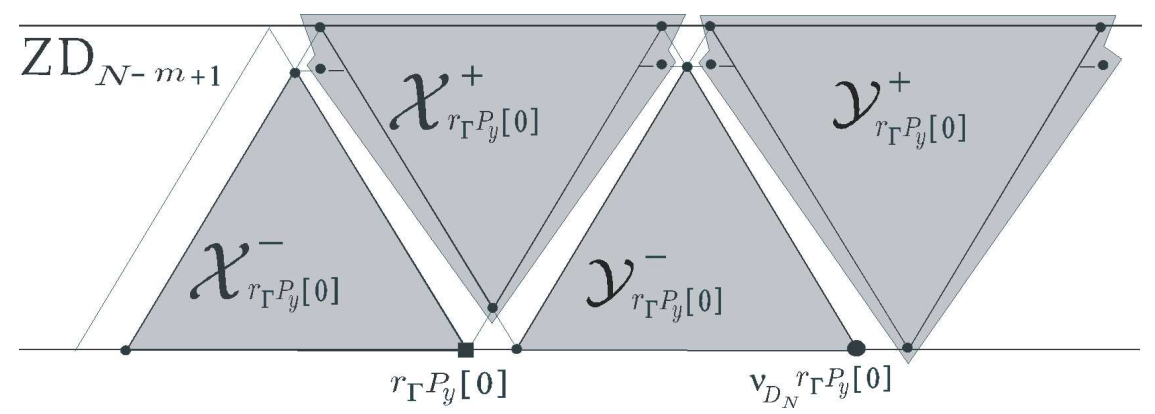

Applying the functor $\Phi=\Phi_{y, i_{0}}: k\left(\mathbb{Z} \mathbf{D}_{N-m+1}\right) \rightarrow k\left(\mathbb{Z} \mathbf{D}_{N}\right)$ to the partition above we get, by 4.10 and 4.11 , that such a partition can be embedded in $k\left(\mathbb{Z} \mathbf{D}_{N}\right)$ as can be seen in the following picture; furthermore, in this picture, we also illustrate the partition $\left\{C_{r_{\Lambda} P_{z}[0]}^{-}[i], C_{r_{\Lambda} P_{z}[0]}^{+}[i]\right.$ : $i \in \mathbb{Z}\}$ of $\mathbb{Z} \mathbf{D}_{N}$ for $z=y_{1}$. 


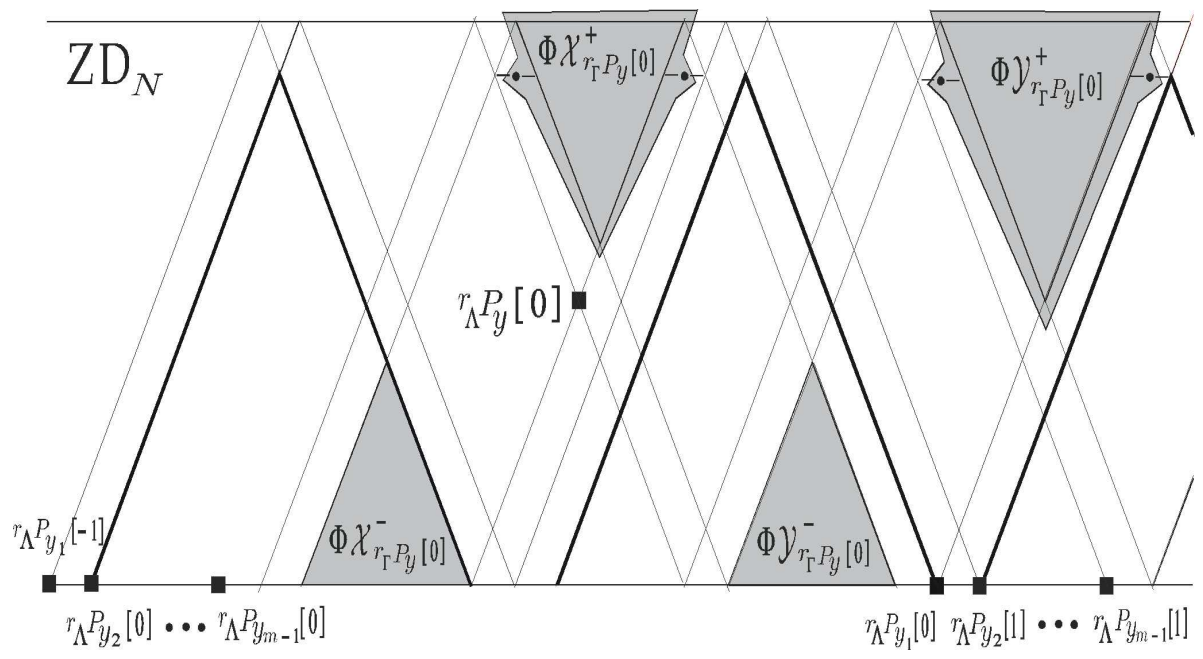

By using the two pictures above, it is not difficult to prove the following lemma.

Lemma (E2) For any $z \in\left\{y_{1}, y_{2}, \cdots, y_{m-1}\right\}$, we have that
(i) $r_{\Lambda} P_{y}[0] \in C_{r_{\Lambda} P_{z}[0]}^{+}$,
(ii) $\Phi\left(C_{r_{\Gamma} P_{y}[0]}^{ \pm}\right) \subseteq C_{r_{\Lambda} P_{z}[0]}^{ \pm}$,
(iii) $r_{\Lambda} P_{w}[0] \in C_{r_{\Lambda} P_{z}[0]}^{-}$for all $w \in\left\{y_{1}, y_{2}, \cdots, y_{m-1}\right\}$.

Now, we are ready to prove (b1) and (b2) in case $\mathcal{C}$ is an elimination cycle of $Q_{\Lambda}$. Let $x$ be a non-initial vertex in $Q_{\Lambda}$, that is, $\partial_{\Lambda}(x) \neq *$.

(b1) Assume that $x$ is $\mathcal{C}$-linked. If $x=y$ then, by (E2) (i), it follows that $r_{\Lambda} P_{x}[0] \in C_{r_{\Lambda} P_{z}[0]}^{+}$, otherwise $x \in\left(Q_{\Gamma}\right)_{0} \backslash\{y\}$; and so, by induction we have $r_{\Gamma} P_{x}[0] \in C_{r_{\Gamma} P_{z}[0]}^{+}$. Hence, from (E2) (ii) and 4.11 (b1), we conclude that $r_{\Lambda} P_{x}[0]=\Phi\left(r_{\Gamma} P_{x}[0]\right) \in \Phi\left(C_{r_{\Gamma} P_{z}[0]}^{+}\right) \subseteq C_{r_{\Lambda} P_{z}[0]}^{+}$; proving (b1).

(b2) Assume that $x$ is $\mathcal{C}$-free. If $x$ is a vertex in the cycle $\mathcal{C}$ then, by (E2) (iii), it follows that $r_{\Lambda} P_{x}[0] \in C_{r_{\Lambda} P_{z}[0]}^{-}$. Suppose that $x \in\left(Q_{\Gamma}\right)_{0}$ 
with $x \neq y$. Then by induction $r_{\Gamma} P_{x}[0] \in C_{r_{\Gamma} P_{z}[0]}^{-}$. Therefore, from (E2) (ii) and 4.11 (b1), we get $r_{\Lambda} P_{x}[0]=\Phi\left(r_{\Gamma} P_{x}[0]\right) \in \Phi\left(C_{r_{\Gamma} P_{z}[0]}^{-} \subseteq\right.$ $C_{r_{\Lambda} P_{z}[0]}^{-}$; proving (b2).

Case 2: Suppose that $\mathcal{C}$ is not an elimination cycle of $Q_{\Lambda}$.

Let $C^{\prime}=x_{m} \leftarrow x_{1} \leftarrow x_{2} \leftarrow \cdots \leftarrow x_{m-1} \leftarrow x_{m}$ be an elimination cycle of $Q_{\Lambda}$ having a chain of elemental cycles $\mathcal{C}=C_{1}^{\prime}, C_{2}^{\prime}, \cdots, C_{\ell-1}^{\prime}, C_{\ell}^{\prime}=$ $C^{\prime}$ as in 6.9 (see the picture below).

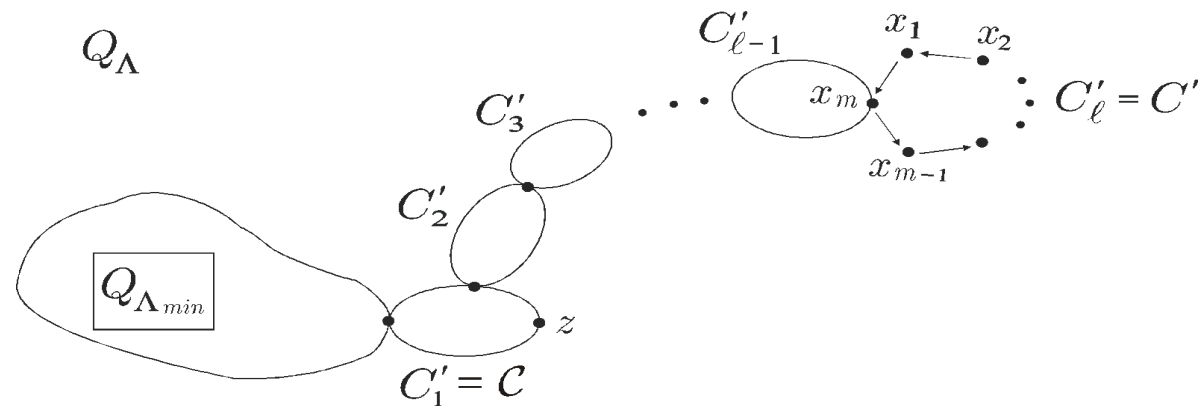

Consider the trivial extension $\Gamma$ of Cartan class $D_{N-m+1}$ obtained from $\Lambda$ by eliminating the cycle $C^{\prime}$. In particular, we have that $x_{m}$ is an insertion vertex of $Q_{\Gamma}$. We assume all the hypothesis needed in 4.11 ; and so, we have a full an faithful functor $\Phi:=\Phi_{x_{m}, i_{0}}$ : $k\left(\mathbb{Z} \mathbf{D}_{N-m+1}\right) \rightarrow k\left(\mathbb{Z} \mathbf{D}_{N}\right)$. Furthermore, we have a partition $\left\{C_{r_{\Gamma} P_{x_{m}}[0]}^{-}[i], C_{r_{\Gamma} P_{x_{m}}[0]}^{+}[i] \quad: \quad i \in \mathbb{Z}\right\}$ of $\mathbb{Z}_{\mathbf{D}_{N-m+1}}$ (see the first picture of this proof). We use the functor $\Phi$, to compare the partition $\left\{C_{r_{\Gamma} P_{z}[0]}^{-}[i], C_{r_{\Gamma} P_{z}[0]}^{+}[i]: i \in \mathbb{Z}\right\}$ of $\mathbb{Z} \mathbf{D}_{N-m+1}$ with the partition $\left\{C_{r_{\Lambda} P_{z}[0]}^{-}[i], C_{r_{\Lambda} P_{z}[0]}^{+}[i]: i \in \mathbb{Z}\right\}$ of $\mathbb{Z} \mathbf{D}_{N}$. Hence, we get the following lemma.

Lemma (E3) With the preceding notations and hypothesis, we have

(i) $\Phi\left(C_{r_{\Gamma} P_{z}[0]}^{ \pm}\right) \subseteq C_{r_{\Lambda} P_{z}[0]}^{ \pm}$,

(ii) $r_{\Lambda} P_{x}[0] \in C_{r_{\Lambda} P_{z}[0]}^{-}$for any vertex $w$ in $C^{\prime}$. 
Now, we are ready to prove (b1) and (b2) in case $\mathcal{C}$ is not an elimination cycle of $Q_{\Lambda}$. Let $x$ be a non-initial vertex in $Q_{\Lambda}$.

(b1) Assume that $x$ is $\mathcal{C}$-linked. So $x \in\left(Q_{\Gamma}\right)_{0}$, and by induction, $r_{\Gamma} P_{x}[0] \in C_{r_{\Gamma} P_{z}[0]}^{+}$. Hence, from (E3) (i) and 4.11 (b1), it follows

$$
r_{\Lambda} P_{x}[0]=\Phi\left(r_{\Gamma} P_{x}[0]\right) \in \Phi\left(C_{r_{\Gamma} P_{z}[0]}^{+}\right) \subseteq C_{r_{\Lambda} P_{z}[0]}^{+} ;
$$

proving (b1)

(b2) Assume that $x$ is $\mathcal{C}$-free. If $x$ is a vertex in the cycle $C^{\prime}$ then, by (E3) (ii), we obtain that $r_{\Lambda} P_{x}[0] \in C_{r_{\Lambda} P_{z}[0]}^{-}$.

Suppose that $x \in\left(Q_{\Gamma}\right)_{0}$ with $x \neq x_{m}$. So, we have by induction that $r_{\Gamma} P_{x}[0] \in C_{r_{\Gamma} P_{z}[0]}^{-}$. Therefore, from (E3) (i) and 4.11 (b1), we get that

$$
r_{\Lambda} P_{x}[0]=\Phi\left(r_{\Gamma} P_{x}[0]\right) \in \Phi\left(C_{r_{\Gamma} P_{z}[0]}^{-}\right) \subseteq C_{r_{\Lambda} P_{z}[0]}^{-} ;
$$

proving the result.

Now, we are in position to prove the main result of this section.

Proof of Theorem 6.8 It is enough to prove (b) since it implies (a) and (c). The proof will be carried out by induction on the number of minimal oriented cycles of $Q_{\Lambda}$. If $\Lambda$ is minimal then from 2.11, 2.12, 2.14 and 2.15 the proof of (b) is complete.

Suppose that $\Lambda$ is not minimal. Let $C^{\prime}=z \leftarrow z_{1} \leftarrow z_{2} \leftarrow$ $\cdots z_{m-1} \leftarrow z$ be an elimination cycle of $Q_{\Lambda}$. Consider the trivial extension $\Gamma$ of Cartan class $\mathbf{D}_{N-m+1}$ obtained from $\Lambda$ eliminating the cycle $C^{\prime}$. We assume all the hypothesis needed in 4.11 ; and so, we have a full an faithful functor

$$
\Phi:=\Phi_{z, i_{0}}: k\left(\mathbb{Z} \mathbf{D}_{N-m+1}\right) \rightarrow k\left(\mathbb{Z} \mathbf{D}_{N}\right) .
$$

Using such a functor, we will compare $r \mathcal{P}\left(\Gamma, \mathbb{Z} \mathbf{D}_{N-m+1}\right)$ with $r \mathcal{P}\left(\Lambda, \mathbb{Z} \mathbf{D}_{N}\right)$. Consider the partition $\left\{C_{r_{\Gamma} P_{z}[0]}^{-}[i], C_{r_{\Gamma} P_{z}[0]}^{+}[i]: i \in \mathbb{Z}\right\}$ of $\mathbb{Z} \mathbf{D}_{N-m+1}$ (see 4.10 (a)). Then, the functor $\Phi$ embeds such a partition in $\mathbb{Z} \mathbf{D}_{N}$ as can be seen in 4.10 (b).

By induction, we have that Theorem 6.8 is true for $\Gamma$; therefore $\pi\left({\widehat{r_{\Gamma} P_{i}}}\right)=r_{\Gamma} P_{i}$ for any vertex $i \in Q_{\Gamma}$, where $\pi: \mathbb{Z D}_{N-m+1} \rightarrow{ }_{S} \Gamma_{\Gamma}$ is the universal covering of ${ }_{S} \Gamma_{\Gamma}$. 
Given a vertex $x$ in $\mathbb{Z} \mathbf{D}_{N-m+1}$, we start by finding a relationship between $h_{\mathbb{Z} \mathbf{D}_{N}}(\Phi(x))$ and $h_{\mathbb{Z} \mathbf{D}_{N-m+1}}(x)$.

Lemma A For any vertex $x$ in $\mathbb{Z} \mathbf{D}_{N-m+1}$, we have that

(a1) $\Phi\left(\mathcal{S}_{x}^{ \pm}\right) \subseteq \mathcal{S}_{\Phi(x)}^{ \pm}$if $x$ and $\tau^{-1} x$ belong to the same component in the partition $\left\{C_{r_{\Gamma} P_{z}[0]}^{-}[i], C_{r_{\Gamma} P_{z}[0]}^{+}[i]: i \in \mathbb{Z}\right\}$ of $\mathbb{Z}_{N-m+1}$;

(a2) $\Phi\left(\mathcal{S}_{r_{\Gamma} P_{z}[0]}^{-}\right) \subseteq \mathcal{S}_{r_{\Lambda} P_{z}[0]}^{-}$;

(a3) if $x=r_{\Gamma} P_{j}[d]$ for $j \in\left(Q_{\Gamma}\right)_{0} \backslash\{z\}$ and $d \in \mathbb{Z}$, then $x$ and $\tau^{-1} x$ belong to the same component in the partition $\left\{C_{r_{\Gamma} P_{z}[0]}^{-}[i]\right.$, $\left.C_{r_{\Gamma} P_{z}[0]}^{+}[i]: i \in \mathbb{Z}\right\}$ of $\mathbb{Z} \mathbf{D}_{N-m+1}$.

(a4) $h_{\mathbb{Z} \mathbf{D}_{N}}(\Phi(x))= \begin{cases}h_{\mathbb{Z} \mathbf{D}_{N-m+1}}(x) & \text { if } x \in C_{r_{\Gamma} P_{z}[0]}^{-}, \\ h_{\mathbb{Z} \mathbf{D}_{N-m+1}}(x)+m-1 & \text { if } x \in C_{r_{\Gamma} P_{z}[0]}^{+} .\end{cases}$

Proof. We apply the full an faithful functor $\Phi=\Phi_{z, i_{0}}$ to the desired partition $\left\{C_{r_{\Gamma} P_{z}[0]}^{-}[i], C_{r_{\Gamma} P_{z}[0]}^{+}[i]: i \in \mathbb{Z}\right\}$ of $\mathbb{Z} \mathbf{D}_{N-m+1}$; afterwards, by 4.11 and 6.1 , we get the items (a1), (a2) and (a4) as can be seen by using the following two pictures.

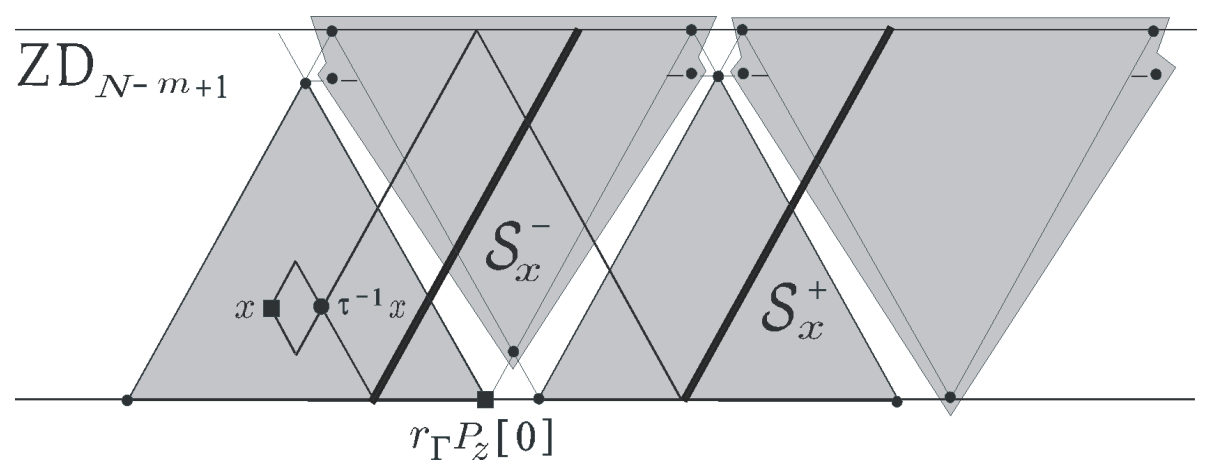




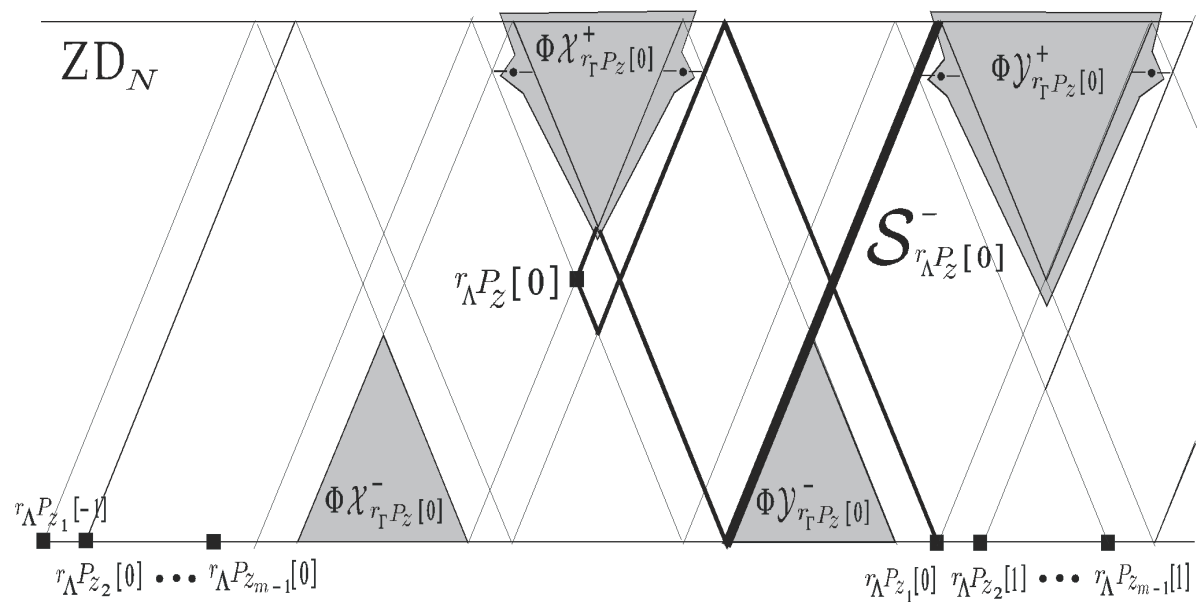

(a3) Denote by $\wp$ to the set of vertices $y$ in $\mathbb{Z} \mathbf{D}_{N-m+1}$ such that $y$ and $\tau^{-1} y$ do not belong to the same component in the partition $\left\{C_{r_{\Gamma} P_{z}[0]}^{-}[i]\right.$,

$\left.C_{r_{\Gamma} P_{z}[0]}^{+}[i]: i \in \mathbb{Z}\right\}$ of $\mathbb{Z} \mathbf{D}_{N-m+1}$. We assert that

$$
\wp=\bigcup_{i \in \mathbb{Z}} \operatorname{Supp}\left(r_{\Gamma} P_{z}[i],-\right) \cup \operatorname{Supp}\left(-, r_{\Gamma} P_{z}[i]\right) .
$$

Indeed, by 1.11 (a), we know the shape in $\mathbb{Z} \mathbf{D}_{N-m+1}$ of $\operatorname{Supp}(-, w)$ and $\operatorname{Supp}(w,-)$ for any vertex $w$ in the bottom border of $\mathbb{Z} \mathbf{D}_{N-m+1}$; and so, our assertion follows from the picture below.

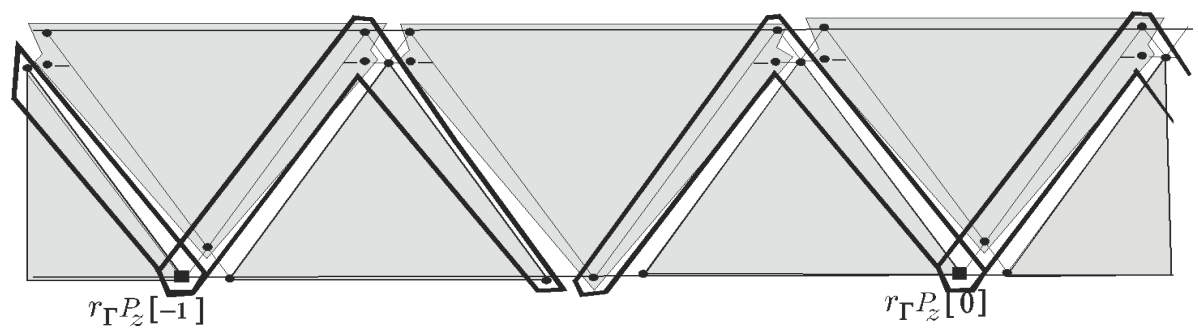

São Paulo J.Math.Sci. 4, 2 (2010), 273-349 
On the other hand, since $j \neq z$ we have that

$$
\underline{\operatorname{Hom}}\left(r_{\Gamma} P_{j}, r_{\Gamma} P_{z}\right)=0=\underline{\operatorname{Hom}}\left(r_{\Gamma} P_{j}, r_{\Gamma} P_{z}\right) .
$$

Thus, by the assertion above, we conclude that $r_{\Gamma} P_{j}[d] \notin \wp$; proving (a3).

Lemma B Let $i \rightarrow j$ be an arrow in $Q_{\Gamma}$ satisfying $\partial_{\Gamma}(i) \neq *$. Then

(b1) if $i \neq z$ and $j \neq z$, then $\Phi\left(\widehat{r_{\Gamma} P_{i}}\right) \in \mathcal{S}_{\Phi\left(\widehat{r_{\Gamma} P_{j}}\right)}^{\partial_{\Lambda}(i)}$ with $h_{\mathbb{Z} \mathbf{D}_{N}}\left(\Phi\left(\widehat{r_{\Gamma} P_{i}}\right)\right)$ $=h_{\Lambda}(i)$;

(b2) if $j=z$ then $\Phi\left(\widehat{r_{\Gamma} P_{i}}\right) \in \mathcal{S}_{r_{\Lambda} P_{z}[0]}$ with $h_{\mathbb{Z} \mathbf{D}_{N}}\left(\Phi\left(\widehat{r_{\Gamma} P_{i}}\right)\right)=h_{\Lambda}(i)$;

(b3) if $i=z$ then $r_{\Lambda} P_{z}[d] \in \mathcal{S}_{\Phi\left(\widehat{r_{\Gamma} P_{j}}\right)}^{\partial_{\Lambda}(z)}$ with $h_{\mathbb{Z} \mathbf{D}_{N}}\left(r_{\Lambda} P_{z}[d]\right)=m-1=$ $h_{\Lambda}(z)$ for some integer $d$.

Proof. (b1) By the definition of $r \mathcal{P}\left(\Gamma, \mathbb{Z} \mathbf{D}_{N-m+1}\right)$, we have that $\widehat{r_{\Gamma} P_{i}} \in \mathcal{S}_{r_{\Gamma} \partial_{j}}^{\partial_{\Gamma}(i)}$ with $h_{\mathbb{Z} \mathbf{D}_{N-m+1}}\left(\widehat{r_{\Gamma} P_{i}}\right)=h_{\Gamma}(i)$. On the other hand, by Lemma A (a1) and (a3), it follows $\Phi\left(\widehat{r_{\Gamma} P_{i}}\right) \in \Phi\left(\mathcal{S}_{r_{\Gamma} P_{j}}^{\partial_{\Gamma}(i)}\right) \subseteq \mathcal{S}_{\Phi\left(r_{\Gamma} P_{j}\right.}^{\partial_{\Lambda}(i)}$ since $\partial_{\Gamma}(i)=\partial_{\Lambda}(i), j \neq z$ and $\widehat{r_{\Gamma} P_{j}}=r_{\Gamma} P_{j}[0]$. In order to prove that $h_{\mathbb{Z} \mathbf{D}_{N}}\left(\Phi\left(\widehat{r_{\Gamma} P_{i}}\right)\right)=h_{\Lambda}(i)$, we consider two cases and use the equality $h_{\mathbb{Z} \mathbf{D}_{N-m+1}}\left(\widehat{r_{\Gamma} P_{i}}\right)=h_{\Gamma}(i)$ that holds by induction.

Case I: $z$ is a vertex in $Q_{\Lambda_{\min }}$. So, in this case, $z$ is the unique vertex which is $C^{\prime}$-linked. In particular, the vertex $i$ is $C^{\prime}$-free; and then, by 6.10 , it follows that $h_{\Gamma}(i)=h_{\Lambda}(i)$. On the other hand, from 6.11 (a), we get that $r_{\Gamma} P_{i}[0] \in C_{r_{\Gamma} P_{z}[0]}^{-}$. Thus, by Lemma A (a4), we obtain that $h_{\mathbb{Z} \mathbf{D}_{N}}\left(\Phi\left(\widehat{r_{\Gamma} P_{i}}\right)\right)=h_{\mathbb{Z} \mathbf{D}_{N-m+1}}\left(\widehat{r_{\Gamma} P_{i}}\right)$. Therefore

$$
h_{\mathbb{Z} \mathbf{D}_{N}}\left(\Phi\left(\widehat{r_{\Gamma} P_{i}}\right)\right)=h_{\mathbb{Z} \mathbf{D}_{N-m+1}}\left({\widehat{r_{\Gamma} P_{i}}}\right)=h_{\Gamma}(i)=h_{\Lambda}(i) .
$$

Case II: $z$ does not belongs to $Q_{\Lambda_{\min }}$. So, the vertex $i$ is either $C^{\prime}$-linked or $C^{\prime}$-free. If $i$ is $C^{\prime}$-free, we get by 6.11 (b2) and 6.10 that $r_{\Gamma} P_{i}[0] \in C_{r_{\Gamma} P_{z}[0]}^{-}$and $h_{\Lambda}(i)=h_{\Gamma}(i)$; and hence

$$
h_{\mathbb{Z} \mathbf{D}_{N}}\left(\Phi\left(\widehat{r_{\Gamma} P_{i}}\right)\right)=h_{\mathbb{Z} \mathbf{D}_{N-m+1}}\left({\widehat{r_{\Gamma} P_{i}}}_{i}=h_{\Gamma}(i)=h_{\Lambda}(i)\right. \text {. }
$$


Assume that the vertex $i$ is $C^{\prime}$-linked. Then, by 6.11 (b1) and 6.10, it follows that $r_{\Gamma} P_{i}[0] \in C_{r_{\Gamma} P_{z}[0]}^{+}$and $h_{\Lambda}(i)=h_{\Gamma}(i)+m-1$. Therefore, $h_{\mathbb{Z} \mathbf{D}_{N}}\left(\Phi\left(\widehat{r_{\Gamma} P_{i}}\right)\right)=h_{\mathbb{Z} \mathbf{D}_{N-m+1}}\left({\widehat{r_{\Gamma} P_{i}}}\right)+m-1=h_{\Gamma}(i)+m-1=h_{\Lambda}(i) ;$ proving (b1).

(b2) By the definition of $\partial_{\Gamma}$ (see 6.4), we have that $\partial_{\Gamma}(i)=-$ since by assumption there is an arrow $i \rightarrow z$ and $z$ is $C^{\prime}$-linked. Then, by using Lemma A (a2), the proof of (b2) follows similarly as the one given in (b1).

(b3) Suppose $i=z$, that is, we have an arrow $z \rightarrow j$ in $Q_{\Gamma}$ with $\partial_{\Gamma}(z) \neq *$. We may assume that $d=0$, since otherwise we apply an appropriate shifting. Then, by induction, we have that $r_{\Gamma} P_{z}[0] \in \mathcal{S}_{r_{\Gamma} P_{j}}^{\partial_{\Gamma}(z)}$. Since $j \neq z$, we conclude, from Lemma A (a3), that $r_{\Gamma} P_{j}[0]$ and $\tau^{-1} r_{\Gamma} P_{j}[0]$ belong to the same component in the partition $\left\{C_{r_{\Gamma} P_{z}[0]}^{-}[i], C_{r_{\Gamma} P_{z}[0]}^{+}[i]: i \in \mathbb{Z}\right\}$ of $\mathbb{Z D}_{N-m+1}$ as can be seen in the following two pictures.

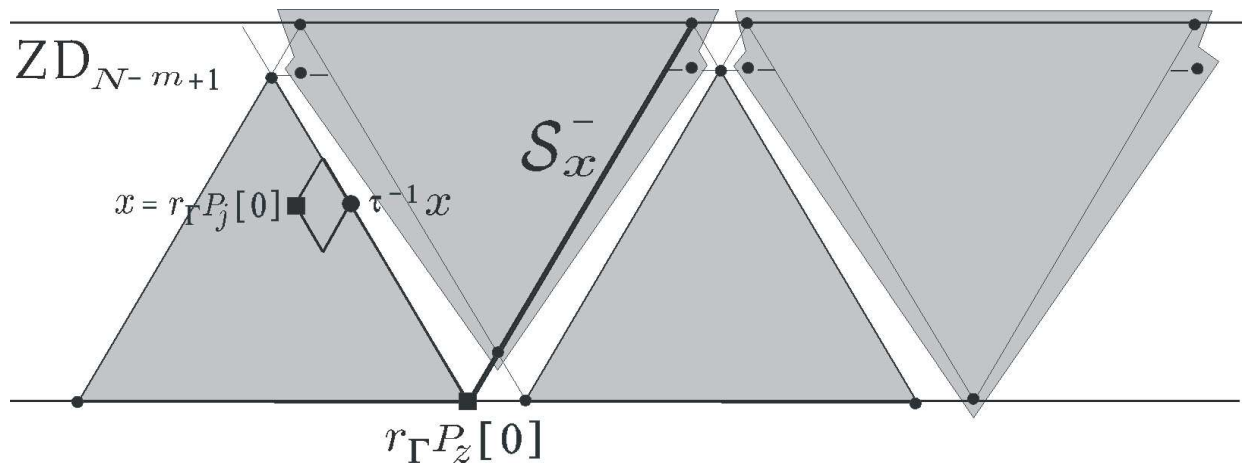




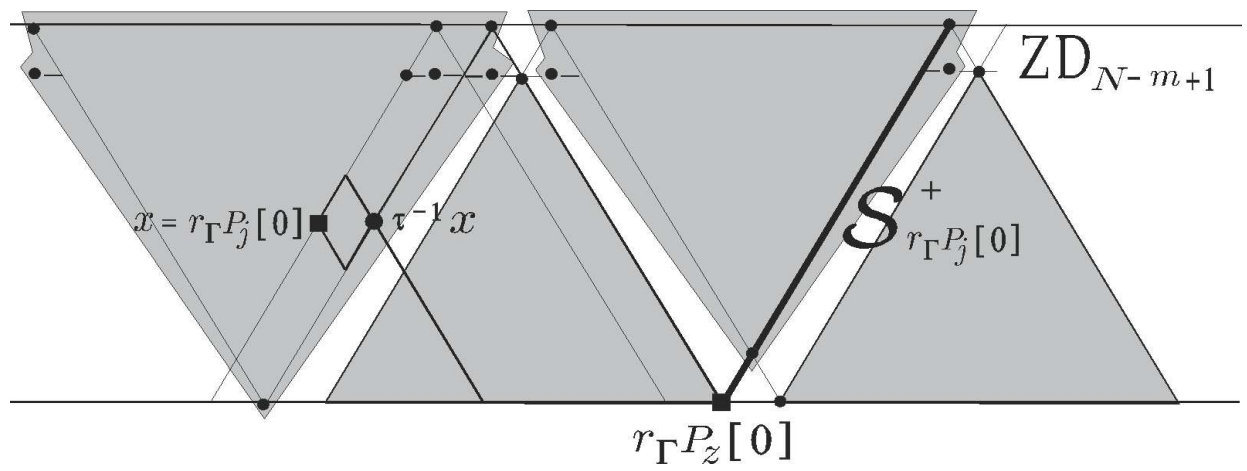

But $h_{\Lambda}(z)=m-1$; and so, by 4.11 , we get that $r_{\Lambda} P_{z}[0] \in \mathcal{S}_{\Phi\left(\widehat{r_{\Gamma} P_{j}}\right)}^{\partial_{\Lambda}(z)}$ with $h_{\mathbb{Z} \mathbf{D}_{N}}\left(r_{\Lambda} P_{z}[0]\right)=m-1=h_{\Lambda}(z)$; proving (b3).

We are now in position to finish the proof of (b) in the Theorem 6.8. For the initial vertices $t$ of $Q_{\Lambda}$, by 6.6 and 6.7 (a), we conclude that $\pi\left(\widehat{r_{\Lambda} P_{t}}\right)=r_{\Lambda} P_{t}$. On the other hand, since $\Phi\left(\widehat{r_{\Gamma} P_{t}}\right)=\Phi\left(r_{\Gamma} P_{t}[0]\right)=$ $r_{\Lambda} P_{t}[0]$, we choose the coordinates of $\widehat{r_{\Lambda} P_{t}}$ in $\mathbb{Z} \mathbf{D}_{N}$ in such a way that $\Phi\left(\widehat{r_{\Gamma} P_{t}}\right)=\widehat{r_{\Lambda} P_{t}}$. So, we assert the following:

$$
\text { (C) } \Phi\left({\widehat{r_{\Gamma} P_{i}}}\right)={\widehat{r_{\Lambda} P_{i}}}^{\text {for any }} i \in\left(Q_{\Gamma}\right)_{0} \backslash\{z\} \text {. }
$$

Indeed, let $i \rightarrow j$ be an arrow in $Q_{\Gamma}$ with $\partial_{\Gamma}(j) \neq *$ and $i \neq z$. Since $\Phi\left(\widehat{r_{\Gamma} P_{t}}\right)=\widehat{r_{\Lambda} P_{t}}$ for $\partial_{\Lambda}(t)=*$, to get $(\mathrm{C})$, it is enough to proof the following:

1) if $j \neq z$ and $\Phi\left(\widehat{r_{\Gamma} P_{j}}\right)=\widehat{r_{\Lambda} P_{j}}$, then $\Phi\left(\widehat{r_{\Gamma} P_{i}}\right)=\widehat{r_{\Lambda} P_{i}}$;

2) if $j=z$ then $\Phi\left(\widehat{r_{\Gamma} P_{i}}\right) \in \mathcal{S}_{r_{\Lambda} P_{z}[0]}^{-}$with $h_{\mathbb{Z} \mathbf{D}_{N}}\left(\Phi\left(\widehat{r_{\Gamma} P_{i}}\right)\right)=h_{\Lambda}(i)$.

But now, the statements 1) and 2) follow from Lemma B items (b1) and (b2); proving (C).

Let now $i$ be any vertex in $Q_{\Lambda}$. We consider several cases as follows.

Case I: Assume that $i \in\left(Q_{\Gamma}\right)_{0} \backslash\{z\}$. Then, by 4.11 (b1), we have that $\Phi\left(r_{\Gamma} P_{i}[0]\right)=r_{\Lambda} P_{i}[0]$. On the other hand, from (C), we know 
that $\Phi\left(\widehat{r_{\Gamma} P_{i}}\right)={\widehat{r_{\Lambda} P_{i}}}_{i}$. Hence $r_{\Lambda} P_{i}[0]=\Phi\left(r_{\Gamma} P_{i}[0]\right)=\Phi\left({\widehat{r_{\Gamma} P_{i}}}^{2}\right)={\widehat{r_{\Lambda} P_{i}}}_{i}$, proving (b) in this case.

Case II: Let $i=z$ and $z \rightarrow j$ be an arrow in $Q_{\Gamma}$. Hence, by $(\mathrm{C})$, we have that $\Phi\left(\widehat{r_{\Gamma} P_{j}}\right)=\widehat{r_{\Lambda} P_{j}}$ since $j \neq z$. Therefore, Lemma B (b3) implies that $r_{\Lambda} P_{z}[d] \in \mathcal{S}_{r_{\Lambda} P_{j}}^{\partial_{\Lambda}(z)}$ with $h_{\mathbb{Z} \mathbf{D}_{N}}^{\partial_{\Lambda}(z)}\left(r_{\Lambda} P_{z}[d]\right)=h_{\Lambda}(z)$; and hence $\widehat{r_{\Lambda} P_{z}}=r_{\Lambda} P_{z}[d]$ for some integer $d$. We may assume that $d=0$, since otherwise we apply an appropriate shifting.

Case III: Suppose that $i \in\left\{z_{1}, z_{2}, \cdots, z_{m-1}\right\}$. We already know that $\widehat{r_{\Lambda} P_{z}}=r_{\Lambda} P_{z}[0]$. Then, from the second picture appearing in the proof of Lemma A, it can be seen that $\widehat{r_{\Lambda} P_{z_{1}}}=r_{\Lambda} P_{z_{1}}[0]$ and so $\widehat{r}_{\Lambda} P_{i}=r_{\Lambda} P_{i}[1]$ for $i=z_{2}, \cdots, z_{m-1}$; proving (b) in this case and ending the proof of the Theorem 6.8.

\section{ACKNOWLEDGMENTS}

The author is very grateful to Professor M.I. Platzeck for helpful discussions and comments. The author is grateful also for the commentaries and suggestions of an anonymous referee, which were very useful in the final version of this paper.

\section{References}

[1] H. Asashiba. The derived Equivalence Classification of RepresentationFinite Selfinjective Algebras. Journal of Algebra 214, 182-221 (1999).

[2] I. Assem. Tilting Theory - an introduction. Topics in algebra. Banach Center publications, volume 26, part 1(1990).

[3] M. Auslander, I. Reiten, S. Smalo. Representation Theory of Artin Algebras. Cambridge University Press, (1995).

[4] Otto Bretscher, Chr. Läser, Chr. Riedtmann. Selfinjective and Simply Connected Algebras. Manuscripta Math. 36, 253-307, (1981).

[5] E. Fernández. Ph.D. Thesis. Universidad Nacional del Sur, Argentina (1999).

[6] E. Fernández and M.I. Platzeck. Presentations of trivial extensions of finite dimensional algebras and a theorem of S. Brenner. Journal of Algebra, 249, 326-344 (2002).

[7] O. Mendoza. Symetric Quasi-Schurian Algebras. Lecture. Notes in pure and applied Mathematics, Marcel Dekker Inc. Octubre 2002, Vol. 224, 99-116.

[8] O. Mendoza and M.I. Platzeck.Embedding of the AR-quiver of an iterated tilted algebra of Dynkin type $\Delta$ in $\mathbb{Z} \Delta$. Journal of algebra 265(2003)247-263. 
[9] O. Mendoza and M.I. Platzeck.Configuration and trivial extension of Dynkin type $\mathbf{A}_{n}$. Journal of algebra 281(2004)167-199.

[10] Chr. Riedtmann. Representation-Finite Selfinjective Algebras of Class $\mathbf{A}_{n}$. LNM 832, 449-520, (1980).

[11] Chr. Riedtmann. Configurations of $\mathbb{Z} \mathbf{D}_{n}$. Journal of Algebra, vol. 82, num. 2, June 1983, 309-327.

[12] Chr. Riedtmann. Representation-Finite Selfinjective Algebras of Class $\mathbf{D}_{n}$. Compositio Math, 49 (1983) 231-282. 